\title{
Extragalactic Peaked-spectrum Radio Sources at Low Frequencies
}

\author{
J. R. Callingham ${ }^{1,2,3}$, R. D. Ekers ${ }^{2}$, B. M. Gaensler ${ }^{1,3,4}$, J. L. B. Line ${ }^{3,5}$, N. Hurley-Walker ${ }^{6}$, E. M. Sadler ${ }^{1,3}$, S. J. Tingay ${ }^{6,7}$, \\ P. J. Hancock ${ }^{3,6}$, M. E. Bell ${ }^{2,3}$, K. S. Dwarakanath ${ }^{8}$, B.-Q. For ${ }^{9}$, T. M. O. Franzen ${ }^{6}$, L. Hindson ${ }^{10}$, M. Johnston-Hollitt ${ }^{10}$, \\ A. D. Kapińska ${ }^{3,9}$, E. Lenc ${ }^{1,3}$, B. McKinley ${ }^{3,5}$, J. Morgan ${ }^{6}$, A. R. Offringa ${ }^{11}$, P. Procopio ${ }^{3,5}$, L. Staveley-Smith ${ }^{3,9}$, R. B. Wayth ${ }^{3,6}$, \\ C. $\mathrm{Wu}^{9}$, and Q. Zheng ${ }^{10}$ \\ ${ }^{1}$ Sydney Institute for Astronomy (SIfA), School of Physics, The University of Sydney, NSW 2006, Australia; joseph.callingham@sydney.edu.au \\ ${ }^{2}$ CSIRO Astronomy and Space Science (CASS), Marsfield, NSW 2122, Australia \\ ${ }^{3}$ ARC Centre of Excellence for All-Sky Astrophysics (CAASTRO), Australia \\ ${ }^{4}$ Dunlap Institute for Astronomy \& Astrophysics, University of Toronto, Toronto, ON, M5S 3H4, Canada \\ ${ }^{5}$ School of Physics, The University of Melbourne, Parkville, VIC 3010, Australia \\ ${ }^{6}$ International Centre for Radio Astronomy Research (ICRAR), Curtin University, Bentley, WA 6102, Australia \\ ${ }^{7}$ Istituto Nazionale di Astrofisica (INAF), Istituto di Radioastronomia, Via Piero Gobetti, Bologna, I-40129, Italy \\ ${ }^{8}$ Raman Research Institute (RRI), Bangalore 560080, India \\ ${ }^{9}$ International Centre for Radio Astronomy Research (ICRAR), The University of Western Australia, Crawley, WA 6009, Australia \\ ${ }^{10}$ School of Chemical \& Physical Sciences, Victoria University of Wellington, Wellington 6140, New Zealand \\ ${ }_{11}$ Netherlands Institute for Radio Astronomy (ASTRON), Dwingeloo, The Netherlands \\ Received 2016 November 22; revised 2016 December 19; accepted 2017 January 4; published 2017 February 17
}

\begin{abstract}
We present a sample of 1483 sources that display spectral peaks between $72 \mathrm{MHz}$ and $1.4 \mathrm{GHz}$, selected from the GaLactic and Extragalactic All-sky Murchison Widefield Array (GLEAM) survey. The GLEAM survey is the widest fractional bandwidth all-sky survey to date, ideal for identifying peaked-spectrum sources at low radio frequencies. Our peaked-spectrum sources are the low-frequency analogs of gigahertz-peaked spectrum (GPS) and compact-steep spectrum (CSS) sources, which have been hypothesized to be the precursors to massive radio galaxies. Our sample more than doubles the number of known peaked-spectrum candidates, and $95 \%$ of our sample have a newly characterized spectral peak. We highlight that some GPS sources peaking above $5 \mathrm{GHz}$ have had multiple epochs of nuclear activity, and we demonstrate the possibility of identifying high-redshift $(z>2)$ galaxies via steep optically thin spectral indices and low observed peak frequencies. The distribution of the optically thick spectral indices of our sample is consistent with past GPS/CSS samples but with a large dispersion, suggesting that the spectral peak is a product of an inhomogeneous environment that is individualistic. We find no dependence of observed peak frequency with redshift, consistent with the peaked-spectrum sample comprising both local CSS sources and high-redshift GPS sources. The $5 \mathrm{GHz}$ luminosity distribution lacks the brightest GPS and CSS sources of previous samples, implying that a convolution of source evolution and redshift influences the type of peaked-spectrum sources identified below $1 \mathrm{GHz}$. Finally, we discuss sources with optically thick spectral indices that exceed the synchrotron self-absorption limit.
\end{abstract}

Key words: galaxies: active - radiation mechanisms: general - radio continuum: general

Supporting material: figure set, tar.gz file

\section{Introduction}

Gigahertz-peaked spectrum (GPS), compact steep spectrum (CSS), and high-frequency peaked (HFP) sources are a class of radio-loud active galactic nuclei (AGNs) that have been argued to be the young precursors to massive radio-loud AGN, such as Centaurus A and Cygnus A (Fanti et al. 1990; O'Dea et al. 1991; Dallacasa et al. 2000; Tinti et al. 2005; KunertBajraszewska et al. 2010). GPS and HFP sources are defined as having a peak in their radio spectra and steep spectral slopes either side of the peak. They are also often found with small linear sizes and low radio polarization fractions (O'Dea et al. 1991). CSS sources are thought to be a related class that has similar properties to GPS and HFP sources, but they have peak frequencies below the traditional gigahertz selection frequencies (Fanti et al. 1990). Hence, the main differentiation between GPS, CSS, and HFP sources is the frequency of the spectral peak and the largest linear size. GPS and HFP sources have linear sizes $\lesssim 1 \mathrm{kpc}$ and peak frequencies of $\sim 1-5 \mathrm{GHz}$, and $\gtrsim 5$ GHz, respectively (O'Dea 1998; Dallacasa et al. 2000). In comparison, CSS sources have linear sizes of $\sim 1-20 \mathrm{kpc}$ and are thought to have the lowest peak frequencies $\lesssim 500 \mathrm{MHz}$, but until recently, low radio frequency observations have been lacking to confirm such a situation (Fanti et al. 1990).

The argument that GPS, CSS, and HFP sources represent the first stages of radio-loud AGN evolution was inferred by very long baseline interferometry (VLBI) observations of these sources, revealing small-scale morphologies reminiscent of large-scale radio lobes of powerful radio galaxies, with two steep-spectra lobes surrounding a flat spectrum core (Phillips \& Mutel 1980; Wilkinson et al. 1994; Stanghellini et al. 1997; Orienti et al. 2006; An et al. 2012). Additional multiepoch VLBI observations measured the motion of the hotspots, providing indirect evidence for ages $\lesssim 10^{5}$ years (Owsianik \& Conway 1998; Polatidis \& Conway 2003; Gugliucci et al. 2005). The "youth" scenario for these sources is further supported by high-frequency spectral break modeling (Murgia et al. 1999; Orienti et al. 2010) and the discovery of the empirical relation between rest frame turnover frequencies and linear size (O'Dea \& Baum 1997; O’Dea 1998). This suggests that HFP sources evolve into GPS sources, which in turn 
evolve into CSS sources, and then finally grow to reach the size of FR I and FR II radio galaxies (Fanaroff \& Riley 1974; Kunert-Bajraszewska et al. 2010).

However, the "frustration" hypothesis, which implies that these sources are not young but are confined to small spatial scales due to unusually high nuclear plasma density (van Breugel et al. 1984; Bicknell et al. 1997), has seen a resurgence in explaining the properties of the GPS, CSS, and HFP population (e.g., Peck et al. 1999; Kameno et al. 2000; Marr et al. 2001; Tingay \& de Kool 2003; Orienti \& Dallacasa 2008; Marr et al. 2014; Callingham et al. 2015; Tingay et al. 2015). The primary reasons that a debate remains about the nature of GPS, CSS, and HFP sources are that there appears to be an overabundance of these sources relative to the number of large radio AGN (Readhead et al. 1996; O'Dea \& Baum 1997; Snellen et al. 2000; An \& Baan 2012), and detailed spectral and morphological studies of individual sources have demonstrated that several of these sources are confined to a small spatial scale due to a dense ambient medium and a cessation of AGN activity (e.g., Peck et al. 1999; Orienti et al. 2010; Callingham et al. 2015). It is also possible that both the "youth" and "frustration" scenarios may apply to the GPS, CSS, and HFP population, since sources with intermittent AGN activity may never break through a dense nuclear medium, but young sources with constant AGN activity could evolve past the inner region of the host galaxy (An \& Baan 2012).

One method that can deduce whether a GPS, CSS, or HFP source is frustrated or young is by identifying whether synchrotron self-absorption (SSA) or free-free absorption (FFA) is responsible for the turnover in the radio spectrum (e.g., Tingay \& de Kool 2003; Marr et al. 2014; Callingham et al. 2015). This is because the turnover in a source's spectrum will likely be dominated by FFA when confined to a small spatial scale by a dense medium (Bicknell et al. 1997; Kuncic et al. 1998). To successfully discriminate between SSA and FFA requires comprehensively sampling the spectrum of the source below the turnover, ideally with the observations below the spectral peak occurring simultaneously (O'Dea et al. 1991; O'Dea \& Baum 1997). Previous studies of samples of GPS, CSS, and HFP sources have often had only a single flux density measurement below the spectral peak and were composed of multiepoch data with sparse frequency sampling, such that differentiations between FFA and SSA have been ambiguous for sources in large samples (e.g., O'Dea 1998; Dallacasa et al. 2000; Snellen et al. 2000, 2002; Edwards \& Tingay 2004; Randall et al. 2011). Other methods of differentiating between SSA and FFA, such as spectral variability (Tingay et al. 2015) or the change in circular polarization over the spectral peak (Melrose 1971), have also suffered from having incomplete multiepoch data below the turnover.

In addition to GPS, CSS, and HFP sources, there have been recent studies of a related class of radio-loud AGN referred to as megahertz-peaked spectrum (MPS) sources (Falcke et al. 2004; Coppejans et al. 2015, 2016). These sources have the same spectral shape as GPS, CSS, and HFP sources, but have an observed turnover frequency below $1 \mathrm{GHz}$. MPS sources are believed to be a combination of nearby CSS sources and GPS and HFP sources at high redshift such that the turnover frequency has shifted below a gigahertz as a result of cosmological evolution (Coppejans et al. 2015). In particular, Coppejans et al. (2015) and Coppejans et al. (2016) have demonstrated that low-radio frequency selection criteria can identify non-beamed sources located at $z>2$ and which appear young because of their small linear size. So far, investigations of MPS sources have been limited to small sections of the sky and moderate sample sizes.

We have entered a new era in radio astronomy where the limitations of small fractional bandwidth at low radio frequencies have been lifted, with the Murchison Widefield Array (MWA; Tingay et al. 2013), the Giant Metrewave Radio Telescope (GMRT; Swarup 1991), and the LOw-Frequency ARray (LOFAR; van Haarlem et al. 2013) now operational. With the all-sky surveys at these facilitates nearing completion, such as the GaLactic and Extragalactic All-sky Murchison Widefield Array (GLEAM; Wayth et al. 2015) survey, the TIFR GMRT Sky Survey (TGSS; Intema et al. 2016), and the LOFAR Multifrequency Snapshot Sky Survey (MSSS; Heald et al. 2015), astronomers now have unprecedented access to the radio sky below $300 \mathrm{MHz}$. While the first surveys in radio astronomy were conducted at low frequencies (e.g., Mills et al. 1958; Edge et al. 1959), MSSS and the GLEAM survey represent a significant step forward in the field because they have surveyed the sky with wide fractional bandwidths and much higher sensitivity. In particular, the GLEAM survey represents the widest continuous fractional bandwidth all-sky survey ever produced, with 20 contemporaneous flux density measurements between 72 and $231 \mathrm{MHz}$ for approximately 300,000 sources in the extragalactic catalog produced from the GLEAM survey (Hurley-Walker et al. 2017). Hence, the GLEAM extragalactic catalog is a rich data set to study sources that have a spectral peak at low frequencies.

The purpose of this paper is to use the GLEAM extragalactic catalog to construct the largest sample of peaked-spectrum sources to date, with contemporaneous observations at and below the spectral peak. Such a statistically significant sample has unparalleled frequency coverage below the turnover of GPS, CSS, and HFP sources, providing a database for a comprehensive spectral comparison of the different absorption models, a test of whether sources with low-frequency spectral peaks are preferentially found at high redshift, and analysis of how many peaked-spectrum sources constitute the wider radioloud AGN at low radio frequencies. Note that in this paper we use the term "peaked-spectrum" to collectively refer to GPS, CSS, HFP, and MPS sources, and the terms "spectral peak" and "spectral turnover" interchangeably.

The relevant surveys used in the source selection, the crossmatching routine, and spectral modeling procedure performed are outlined in Sections 2, 3, and 4, respectively. In Section 5 we discuss the selection criteria implemented to identify peaked-spectrum sources. Comparisons of the identified peaked-spectrum sources to known GPS, CSS, and HFP sources, and Ultra-steep-spectrum (USS) sources, are presented in Sections 6 and 7, respectively. Relevant observed and intrinsic spectral features of the peaked-spectrum samples are outlined in Section 8. Finally, we introduce and debate the nature of sources with radio spectra near the limit of SSA in Section 9. In this paper we adopt the standard lambda cold dark matter cosmological model, with parameters $\Omega_{\mathrm{M}}=0.27$, $\Omega_{\Lambda}=0.73$, and the Hubble constant $H_{0}=70 \mathrm{~km} \mathrm{~s}^{-1} \mathrm{Mpc}^{-1}$ (Hinshaw et al. 2013). 


\section{Descriptions of the Surveys Used in the Selection of Peaked-spectrum Sources}

The sensitivity and frequency coverage of the surveys used for selecting peaked-spectrum sources affect the type of sources identified. Most previous studies (e.g., Fanti et al. 1990; O'Dea et al. 1991; Snellen et al. 1998; Stanghellini et al. 1998; Dallacasa et al. 2000; Kunert-Bajraszewska et al. 2010) used surveys that observed the sky at a single frequency around or above $1 \mathrm{GHz}$. Since the inverted spectrum below the spectral turnover distinguishes a peaked-spectrum source, the lowfrequency survey used for selection dictates the type of sources identified, while higher frequency surveys are used to confirm the turnover and measure the spectral slope in the optically thin regime. Additionally, it is ideal when the higher frequency surveys have better sensitivities compared to the low-frequency survey to ensure that the selected peaked-spectrum sample has a completeness set only by the low-frequency data.

The GLEAM extragalactic catalog represents a significant advance in selecting peaked-spectrum sources, since it is constituted of sources that were contemporaneously surveyed with the widest fractional radio bandwidth to date, with 20 flux density measurements between 72 and $231 \mathrm{MHz}$. With this frequency coverage, selection below and at the spectral peak can be performed solely using one survey. To increase the validity of the detection of the peak in the spectra of these sources, to remove flat-spectrum sources, and to measure the slope above the turnover, we also use the NRAO VLA Sky Survey (NVSS; Condon et al. 1998) and the Sydney University Molonglo Sky Survey (SUMSS; Bock et al. 1999; Mauch et al. 2003). Since the combination of NVSS and SUMSS cover the entire GLEAM survey and are an order of magnitude more sensitive, this study is sensitive to peaked-spectrum sources that peak anywhere between $72 \mathrm{MHz}$ and $843 \mathrm{MHz} / 1.4 \mathrm{GHz}$. Examples of the types of peaked-spectrum sources that this study and previous studies identify are highlighted in Figure 1. Details of the surveys used to select peaked-spectrum sources in this study are provided below.

\subsection{GLEAM Survey}

The GLEAM survey was formed from observations conducted by the MWA, which surveyed the sky between 72 and $231 \mathrm{MHz}$ from 2013 August to 2014 July (Wayth et al. 2015). The MWA is a low radio frequency aperture array that is composed of 128 32-dipole antenna "tiles" spread over $\mathrm{a} \approx 10 \mathrm{~km}$ area in Western Australia (Tingay et al. 2013). The extragalactic catalog formed from the GLEAM survey consists of 307,455 sources south of decl. $+30^{\circ}$, excluding Galactic latitudes $|b|<10^{\circ}$, the Magellanic clouds, within $9^{\circ}$ of Centaurus A, and a 859 square degree section of the sky centered at R.A. $23 \mathrm{hr}$ and decl. $+15^{\circ}$. The positions of the sources reported are accurate to within $\approx 30^{\prime \prime}$, and the catalog is $\approx 90 \%$ complete at $0.16 \mathrm{Jy}$ (Hurley-Walker et al. 2017). The sources in the catalog have 20 flux density measurements between 72 and $231 \mathrm{MHz}$, mostly separated by $7.68 \mathrm{MHz}$.

While the data reduction process that was performed to produce the GLEAM extragalactic catalog is discussed in detail in Hurley-Walker et al. (2017), we summarize the details here considering the importance of the GLEAM extragalactic catalog to this study. At seven independent declination settings, the GLEAM survey employed a two-minute "snapshot" observing mode. COTTER (Offringa et al. 2015) was used to

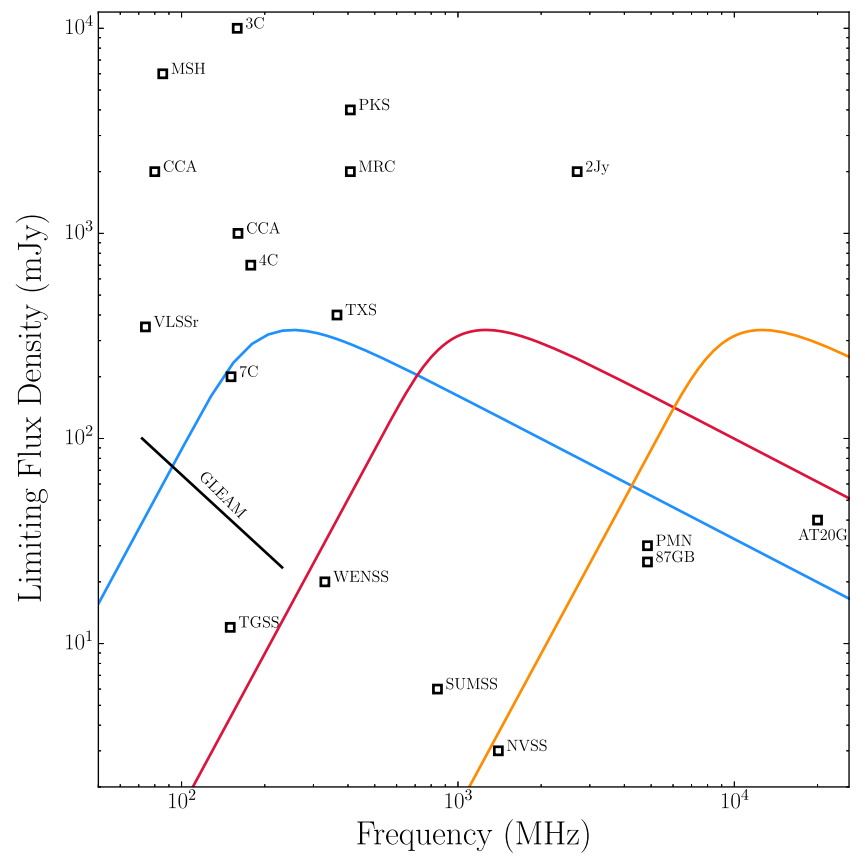

Figure 1. The different frequencies and limiting sensitivities for the major radio surveys. The GLEAM survey is shown as a black line because it has variable limiting sensitivities between 72 and $231 \mathrm{MHz}$. The blue, red, and orange curves represent the SSA spectra of peaked-spectrum sources that peak at 200,1000, and 10,000 MHz, respectively. This figure shows that this study is sensitive to the peaked-spectrum sources portrayed by the blue spectrum, but not to previously identified peaked-spectrum sources portrayed by the red or orange spectra. The other plotted surveys not previously introduced are as follows: Mills, Slee, and Hill (MSH; Mills et al. 1958, 1960, 1961) survey, Cambridge 3C (Edge et al. 1959), 4C (Pilkington \& Scott 1965), and 7C (Hales et al. 2007) surveys, Culgoora circular array (CCA; Slee 1995) survey, VLA Low-frequency Sky Survey redux (VLSSr; Lane et al. 2014) survey, Texas survey (TXS; Douglas et al. 1996), Parkes (PKS; Ekers 1969) survey, Molonglo Reference Catalogue (MRC; Large et al. 1981, 1991), Westerbork Northern Sky Survey (WENSS; Rengelink et al. 1997), 2 Jy survey (Wall \& Peacock 1985), Parkes-MIT-NRAO (PMN; Gregory et al. 1994; Wright et al. 1994) survey, MIT-Green Bank 5 GHz (87GB; Gregory \& Condon 1991) survey, and Australia Telescope $20 \mathrm{GHz}$ (AT20G; Murphy et al. 2010) survey.

process the visibility data, and any radio frequency interference was excised by the AOFLAGGER algorithm (Offringa et al. 2012). For the five instantaneous observing bandwidths of $30.72 \mathrm{MHz}$, which are observed approximately two minutes apart, an initial sky model was produced by observing bright calibrator sources. WSCLEAN (Offringa et al. 2014) was used to perform the imaging of the observations, implementing a "robust" parameter of -1.0 (Briggs 1995), which is close to uniform weighting. Each snapshot observation had multifrequency synthesis applied across the instantaneous bandwidth, and was then CLEANed to the first negative CLEAN component. A self-calibration loop was then applied to each of the images. The shallowly CLEANed $30.72 \mathrm{MHz}$ bandwidth observations were divided into four $7.68 \mathrm{MHz}$ sub-bands and jointly CLEANed, resulting in a rms of $\approx 100-\approx 20 \mathrm{mJy}^{\text {beam }^{-1}}$ for 72 to $231 \mathrm{MHz}$, respectively.

The $408 \mathrm{MHz}$ Molonglo Reference Catalogue (MRC; Large et al. 1981, 1991), scaled to the respective frequency, was used to set an initial flux density scale for the images and to apply an astrometric correction. The snapshots for an observed declination strip were mosaicked, with each snapshot weighted by the square of the primary beam response. Owing to inaccuracies in the primary beam model, the remaining declination dependence in the flux density scale was corrected using the Very Large 
Array Low-frequency Sky Survey Redux (VLSSr; Lane et al. 2014), MRC, and NVSS, and to place the survey on the Baars et al. (1977) flux density scale. It is estimated that the flux density calibration is internally accurate to within $2 \%-3 \%$ and accurate to $8 \%-13 \%$ when comparing the GLEAM flux densities to other surveys (Hurley-Walker et al. 2017).

For each mosaic, a deep wideband image covering $170-231 \mathrm{MHz}$ was formed, with a resolution of $\approx 2^{\prime}$, to provide a higher signal-to-noise ratio $(\mathrm{S} / \mathrm{N})$ and more accurate source positions than what can be attained from a single $7.68 \mathrm{MHz}$ sub-band image. The sources in this wideband image were then convolved with the appropriate synthesized beam and used as priors for characterizing the flux density of the sources at each of the sub-band frequencies, using the source finding and characterization program AEGEAN ${ }^{12}$ v1.9.6 (Hancock et al. 2012).

\subsection{NVSS}

NVSS is a $1.4 \mathrm{GHz}$ continuum survey that was conducted by the Very Large Array (VLA) between 1993 and 1996 (Condon et al. 1998). It covers the entire sky north of a declination of $-40^{\circ}$ and at a resolution of $\approx 45^{\prime \prime}$. The $\mathrm{nn}$ catalog produced from the survey has a total of $1,810,672$ sources, which is $100 \%$ complete above $4 \mathrm{mJy}$. The positions of the sources are accurate to within $1^{\prime \prime}$.

\subsection{SUMSS}

SUMSS is a continuum survey designed to have similar frequency, resolution, and sensitivity to NVSS, but to cover the sky below the declination limit of NVSS. SUMSS was conducted by the Molonglo Observatory Synthesis Telescope (MOST; Mills 1981; Robertson 1991) at $843 \mathrm{MHz}$ between 1997 and 2003, covering the sky south of a declination of $-30^{\circ}$, excluding Galactic latitudes below $10^{\circ}$ (Bock et al. 1999; Mauch et al. 2003). The resolution of the survey varied with declination $\delta$ as $45^{\prime \prime} \times 45^{\prime \prime} \operatorname{cosec}|\delta|$. The catalog consists of 211,063 sources, with a limiting peak brightness of $6 \mathrm{mJy}$ beam $^{-1}$ for sources with declinations below $-50^{\circ}$, and $10 \mathrm{mJy}$ beam $^{-1}$ for sources with declinations above $-50^{\circ}$. Positions in the catalog are accurate to within $1^{\prime \prime}-2^{\prime \prime}$ for sources with flux densities greater than $20 \mathrm{mJy} \mathrm{beam}^{-1}$, and are always better than $10^{\prime \prime}$. The survey is believed to be $100 \%$ complete above $\approx 8 \mathrm{mJy}$ south of a declination of $-50^{\circ}$, and above $\approx 18 \mathrm{mJy}$ for sources between declinations of $-50^{\circ}$ and $-30^{\circ}$.

\subsection{Additional Radio Surveys}

While GLEAM, NVSS, and SUMSS were the only surveys used for selecting peaked-spectrum sources, once a peakedspectrum source was identified, it was cross-matched to other all-sky radio surveys that covered any part of the GLEAM survey region. This included the $74 \mathrm{MHz}$ VLSSr, $408 \mathrm{MHz}$ MRC, and the Australia Telescope $20 \mathrm{GHz}$ (AT20G; Murphy et al. 2010) survey. These additional surveys where not used in any of the following spectral modeling, unless explicitly stated otherwise, but will be shown in any spectral energy distributions to help identify if a spectral fit to the GLEAM and NVSS/ SUMSS data is accurate.

We note that at the time of writing, the $150 \mathrm{MHz}$ TGSSAlternative data release 1 (TGSS-ADR1; Intema et al. 2016)

\footnotetext{
${ }^{12}$ https://github.com/PaulHancock/Aegean
}

was released and undergoing review, including refining the uniformity of its flux density scale. The identified peakedspectrum sources were also cross-matched to TGSS-ADR1, but TGSS-ADR1 was not used for the selection of peakedspectrum sources despite a significant improvement in sensitivity and resolution compared to the GLEAM survey. This is largely because TGSS-ADR1 only surveyed the sky at a single frequency with a comparatively small bandwidth of $17 \mathrm{MHz}$, thus increasing the potential for a sample to be biased by variable sources.

\section{Cross-matching Routine}

The Positional Update and Matching Algorithm (PUMA; Line et al. 2016) was used to assess the probability of a crossmatch between the GLEAM extragalactic catalog and NVSS/ SUMSS. PUMA is an open source cross-matching software, ${ }^{13}$ specifically designed for matching low-frequency radio $(\lesssim 1 \mathrm{GHz})$ catalogs that have varying resolutions. It implements a Bayesian positional matching approach that uses catalog source density, sky coverage, and positional errors as a prior to calculate the probability of a true match for any cross-match (Budavári \& Szalay 2008).

As the surveying telescopes used to create the all-sky catalogs have differing resolutions, multiple matches are common when cross-matching the different catalogs (see e.g., Carroll et al. 2016). Confused matches can manifest in two different ways: multiple sources from a higher resolution catalog appear to match a single source from a lower resolution catalog, when really only one source truly matches; a lower resolution catalog is blending multiple components together, and so multiple sources from a higher resolution catalog do truly match a single lower resolution source.

In this work, the GLEAM extragalactic catalog was used as the base catalog, and was individually cross-matched to SUMSS and NVSS, with an angular cutoff of $2^{\prime} 20^{\prime \prime}$, which is approximately the FWHM of the MWA beam in the wideband image. All possible matches were retained and the results combined to create groups of possible cross-matches to each GLEAM source. Within each group, the positional probability of a true match was calculated for each cross-match (a combination of sources that only includes one source from each catalog). Using these positional results, we then selected the sources that PUMA assigned as isolated, implying only one source from each catalog lay within $2^{\prime} 20^{\prime \prime}$. These cases were accepted if all matched sources lay within $1^{\prime} 10^{\prime \prime}$ of the GLEAM source position or if the positional probability of the cross-match was $>0.99$. We excluded cases where multiple sources were matched to a GLEAM source, since we are interested in peaked-spectrum sources, which are defined to be unresolved at the resolution of the surveys used in this study. Additional details about the cross-matching of the GLEAM sample are presented in Section 5.

\section{Spectral Modeling Procedure}

Selecting and assessing the spectral properties of peakedspectrum sources requires fitting their spectra. The parameter values of various models fit in this study were assessed using the Bayesian model inference routine outlined in Callingham et al. (2015). In summary, a Markov chain Monte Carlo

\footnotetext{
${ }^{13}$ https://github.com/JLBLine/PUMA
} 
algorithm was used to sample the posterior probability density functions of the various model parameters. The parameter values were accepted when the applied Gaussian likelihood function was maximized under physically sensible uniform priors. The affine-invariant ensemble sampler of Goodman \& Weare (2010), via the Python package emcee (ForemanMackey et al. 2013), was implemented. We used the simplex algorithm to direct the walkers to the maximum of the likelihood function (Nelder \& Mead 1965).

When fitting within the GLEAM band, we assumed that the flux density measurements were independent and the uncertainties were Gaussian. However, the known correlation between the sub-band flux densities within the GLEAM band (see Section 5.4 of Hurley-Walker et al. 2017) had to be modeled when GLEAM data were fit simultaneously with other surveys, to ensure that any spurious trends present in the GLEAM flux density measurements did not influence any physical relations. It is not possible to calculate the exact form of the covariance matrix that would describe the correlation between the GLEAM points, but it can be approximated using Gaussian processes with a Matérn covariance function (Rasmussen \& Williams 2006). The Matérn covariance function produces a stronger correlation between flux density measurements closer in frequency space than farther away, as is physically expected for the GLEAM correlation since it largely arises from a complex interaction of multifrequency CLEAN, self-calibration, and side-lobe confusion (Franzen et al. 2016; Hurley-Walker et al. 2017).

\subsection{Spectral Models}

In this study, the spectra of sources are fit with four different spectral models to help select and characterize peakedspectrum sources. First, we use the standard nonthermal power-law model of the form

$$
S_{\nu}=a \nu^{\alpha},
$$

where $a$, in Jy, characterizes the amplitude of the synchrotron spectrum, $\alpha$ is the synchrotron spectral index, and $S_{\nu}$ is the flux density at frequency $\nu$, in MHz. Since the GLEAM survey has a large fractional bandwidth and radio sources are known to show curvature in their observed spectra when closely sampled (Blundell et al. 1999), we also fit the curved power-law model characterized as

$$
S_{\nu}=a \nu^{\alpha} e^{q(\ln \nu)^{2}},
$$

where $q$ parametrizes the spectral curvature and $\nu_{\mathrm{p}}=e^{-\alpha / 2 q}$ is the frequency at which the spectrum peaks. Significant curvature is represented by values of $|q|>0.2$, and the spectral curvature flattens toward a standard power law as $q$ approaches zero. While such a parameterization of curvature might not seem physically motivated, Duffy \& Blundell (2012) have shown that $q$ can be directly related to the magnetic field strength, energy density, and electron density of lobedominated sources.

Additionally, the following generic curved model was used to characterize the entire spectrum of a peaked-spectrum source:

$$
S_{\nu}=\frac{S_{\mathrm{p}}}{\left(1-e^{-1}\right)}\left(1-e^{-\left(\nu / \nu_{\mathrm{p}}\right)^{\alpha_{\text {thin }}-\alpha_{\text {thick }}}}\right)\left(\frac{\nu}{\nu_{\mathrm{p}}}\right)^{\alpha_{\text {thick }}},
$$

where $\alpha_{\text {thick }}$ and $\alpha_{\text {thin }}$ are the spectral indices in the optically thick and optically thin regimes of the spectrum, respectively. $S_{\mathrm{p}}$ is the flux density at the frequency $\nu_{\mathrm{p}}$ (Snellen et al. 1998). When $\alpha_{\text {thick }}=2.5$, this model reduces to a homogeneous SSA source. Equation (3) does not assume the underlying absorption mechanism is SSA or FFA, but does require that the slope of the spectrum above and below the spectral peak be modeled by a power law (similar to e.g., Bicknell et al. 1997). We note that this model is only used to describe the spectrum of a source, not to assess whether SSA or FFA is responsible for the turnover.

In rare cases, the spectrum of a source is not well modeled by a turnover with power-law slopes on either side. To describe the spectra of these complex sources, it is assumed that the particle population producing the nonthermal power-law spectrum is surrounded by a homogeneous ionized screen of plasma such that

$$
S_{\nu}=a \nu^{\alpha} e^{\left(\nu / \nu_{\mathrm{p}}\right)^{-2.1}} .
$$

This homogeneous FFA model is used to model the spectra of such sources because it produces an exponential attenuation below the spectral peak, as opposed to the power-law relations described by Equation (3). The FFA model and the spectra of the sources that the model is used to describe are discussed in more detail in Section 9.

\section{Peaked-spectrum Source Selection Criteria}

To produce a complete and reliable sample, we require a selection criterion that is effective in selecting a particular type of source and that is well-defined such that any introduced bias is easily quantified. For identifying peaked-spectrum sources, the selection criterion involves characterizing whether the distinguishing feature of a spectral peak occurs in a source's spectrum.

Previous studies have made the assessment of a spectral peak in radio color-color phase space (e.g., Sadler et al. 2006; Massardi et al. 2011), where radio color-color phase space is defined by the spectral index derived between two high frequencies $\alpha_{\text {high }}$ and the spectral index derived between two lower frequencies $\alpha_{\text {low. }}$. If $\alpha_{\text {low }}$ had an opposite sign to $\alpha_{\text {high }}$, it was assumed that a peak occurs in the frequency range somewhere between the frequencies in which $\alpha_{\text {low }}$ and $\alpha_{\text {high }}$ were derived. We also use radio color-color space for identifying sources that peak somewhere between the end of the GLEAM frequency coverage and the start of the frequency coverage of SUMSS/NVSS. However, due to the large fractional bandwidth of the GLEAM survey, sources that have a turnover between 72 and $231 \mathrm{MHz}$ could be missed in radio color-color phase space since a power law does not accurately describe their spectra. Therefore, we also identify peakedspectrum sources through a direct measurement of their curvature in the GLEAM band. This is outlined below in Sections 5.1 and 5.2. 
Table 1

A Summary of the Applied Selection Criteria and the Number of Sources that Remained After Each Stage of Selection

\begin{tabular}{|c|c|c|}
\hline $\begin{array}{l}\text { Selection } \\
\text { Step }\end{array}$ & Selection Criterion & $\begin{array}{c}\text { Number } \\
\text { of } \\
\text { Sources }\end{array}$ \\
\hline 0 & $\begin{array}{l}\text { Total GLEAM extragalactic catalog } \\
\qquad\left(|b| \geqslant 10^{\circ}, \delta \leqslant+30^{\circ}\right)\end{array}$ & 307,456 \\
\hline 1 & $\begin{array}{l}\text { Unresolved in the GLEAM wideband image } \\
\qquad a b /\left(a_{\mathrm{psf}} b_{\mathrm{psf}}\right) \leqslant 1.1\end{array}$ & 210,365 \\
\hline 2 & $\delta \geqslant-80^{\circ}$ & 208,595 \\
\hline 3 & $S_{200 \mathrm{MHz}, \text { wide }} \geqslant 0.16 \mathrm{Jy}$ & 98,329 \\
\hline 4 & $\begin{array}{l}\text { Sources with } 8 \text { or more GLEAM flux density } \\
\text { measurements with a } S / N \geqslant 3\end{array}$ & 96,698 \\
\hline 5 & NVSS and/or SUMSS counterpart & 96,628 \\
\hline 6 & Peaked-spectrum selection & 1483 \\
\hline $6 a$ & $\begin{array}{l}\text { High-frequency soft sample } \\
\alpha_{\text {low }} \geqslant 0.1 \text { and } \alpha_{\text {high }} \leqslant-0.5\end{array}$ & 207 \\
\hline $6 b$ & $\begin{array}{l}\text { High-frequency hard sample } \\
\alpha_{\text {low }} \geqslant 0.1 \text { and }-0.5<\alpha_{\text {high }} \leqslant 0\end{array}$ & 506 \\
\hline $6 c$ & $\begin{array}{l}\text { GPS sample } \\
\alpha_{\text {low }} \geqslant 0.1 \text { and } \alpha_{\text {high }}>0\end{array}$ & 261 \\
\hline $6 d$ & $\begin{array}{l}\text { Low-frequency soft sample } \\
\alpha_{\text {low }}<0.1, \alpha_{\text {high }} \leqslant-0.5 \\
\quad 72 \mathrm{MHz} \leqslant \nu_{\mathrm{p}} \leqslant 231 \mathrm{MHz}, q \leqslant-0.2 \\
\quad \text { and } \Delta q \leqslant 0.2\end{array}$ & 394 \\
\hline $6 e$ & $\begin{array}{l}\text { Low-frequency hard sample } \\
\alpha_{\text {low }}<0.1 \text {, } \\
\quad-0.5<\alpha_{\text {high }} \leqslant 0,72 \mathrm{MHz} \leqslant \nu_{\mathrm{p}} \leqslant 231 \mathrm{MHz} \text {, } \\
\quad q \leqslant-0.2 \text {, and } \Delta q \leqslant 0.2\end{array}$ & 115 \\
\hline
\end{tabular}

Note. Italicized numbers indicate the subset of sources selected from the previous non-italicized number. The details of the selection process are discussed in Section 5. With regard to the peaked-spectrum samples, "high frequency" refers to sources with a spectral peak above a frequency of $\approx 180 \mathrm{MHz}$, while "low frequency" refers to sources with a spectral peak below a frequency of $\approx 180 \mathrm{MHz} . b$ and $\delta$ represent galactic latitude and declination, respectively.

\subsection{Sky Area, Resolution, and Flux Density Limits}

Before making a distinction based on the spectral properties of a source, we must first make resolution, cross-matching, and flux density cuts to the GLEAM extragalactic catalog to ensure that a reliable and complete peaked-spectrum sample with wellunderstood biases is derived. The selection criteria employed are summarized in Table 1 and detailed below.

1. Any source that is resolved in the GLEAM wideband image, centered on $200 \mathrm{MHz}$, was eliminated since peaked-spectrum sources are found to have small spatial scales. The wideband image was used to perform this cut because it achieves the highest resolution of the GLEAM survey of $\approx 2^{\prime}$, and all sources in the GLEAM survey are found within the wideband image. We determined whether a source was resolved in the wideband image by the criterion $a b /\left(a_{\mathrm{psf}} b_{\mathrm{psf}}\right) \leqslant 1.1$, where $a, b, a_{\mathrm{psf}}$, and $b_{\text {psf }}$ are the semimajor and semiminor axes of a source and the point-spread function, respectively. While this resolution limit depends on the $\mathrm{S} / \mathrm{N}$, the flux density cut outlined in step 3 below ensures that this step has not removed potential peaked-spectrum sources with a low signal-to-noise ratio. This resolution cut reduces the total GLEAM extragalactic catalog by approximately onethird to 208,595 sources.
2. Sources that are located within $10^{\circ}$ of the south celestial pole were removed because uncertainties in the GLEAM flux density scale were greater than $80 \%$, and uncertainties in the GLEAM positions were greater than $1^{\prime}$. Such large uncertainties are mostly due to blurring of the source that results from mosaicking many hours of data with different ionospheric conditions (Hurley-Walker et al. 2017). This decreased the sample by an additional 1770 sources, or $0.8 \%$. We note that sources between declinations of $-72^{\circ}$ and $-80^{\circ}$ have larger GLEAM systematic uncertainties than the rest of the survey to reflect the larger uncertainty in the flux density scale for this part of the sky.

3. A flux density cut was made to provide a reliable peakedspectrum sample and to evaluate its completeness. The GLEAM extragalactic catalog is estimated to be $\approx 90 \%$ complete and $99.98 \%$ reliable at $0.16 \mathrm{Jy}$ based on the wideband image. Therefore we only investigated sources that had $S_{200 \mathrm{MHz} \text {,wide }} \geqslant 0.16 \mathrm{Jy}$, where $S_{200 \mathrm{MHz} \text {,wide }}$ is the flux density in the wideband image. Imposing this flux density limit also guarantees that the sample is only formed from sources with $\mathrm{S} / \mathrm{Ns}$ greater than 20, limiting the impact of classical confusion on the spectra of the sources (Murdoch et al. 1973). The sample was approximately halved to 98,329 sources after this flux density cut.

4. The previous flux density cut selects a reliable sample from the wideband image but does not account for local variations in the noise within the sub-band images. Large variations in the local rms noise with frequency in the GLEAM survey are often due to calibration issues near bright sources or the edge of the survey. Additionally, sources with low $\mathrm{S} / \mathrm{N}$ will suffer from non-Gaussianity in their uncertainties (Hurley-Walker et al. 2017). Since accurate spectra across the entirety of the GLEAM band are needed to reliably select peaked-spectrum sources, we required that a sub-band flux density have a $S / N \geqslant 3$ to be used in the spectral fitting. If a source was left with seven or fewer GLEAM flux density measurements, which represents less than a third of the total GLEAM bandwidth, it was excluded from the sample. This step removed 1739 sources, $\approx 1.8 \%$ of the sources from the previous step.

5. The remaining sources were cross-matched to NVSS and SUMSS using the cross-matching routine outlined in Section 3. Since NVSS and SUMSS are over two orders of magnitude more sensitive than the GLEAM survey, $99.93 \%$ of the sample have a NVSS/SUMSS counterpart. The 70 sources that do not have a counterpart are retained for follow-up investigations. We note that GLEAM sources that are located between declinations of $-30^{\circ}$ and $-40^{\circ}$ have two counterparts because NVSS and SUMSS overlap by $10^{\circ} \mathrm{decl}$.

\subsection{Spectral Classification}

At this step in the selection process, it is possible to define the position of the sources in a radio color-color phase space that is characterized from 72 to $843 \mathrm{MHz} / 1.4 \mathrm{GHz}$. Using the modeling procedure detailed in Section 4, we fit Equation (1) to the 20 GLEAM flux density points to derive the low-frequency spectral index $\alpha_{\text {low }}$. Similarly, the high-frequency spectral 


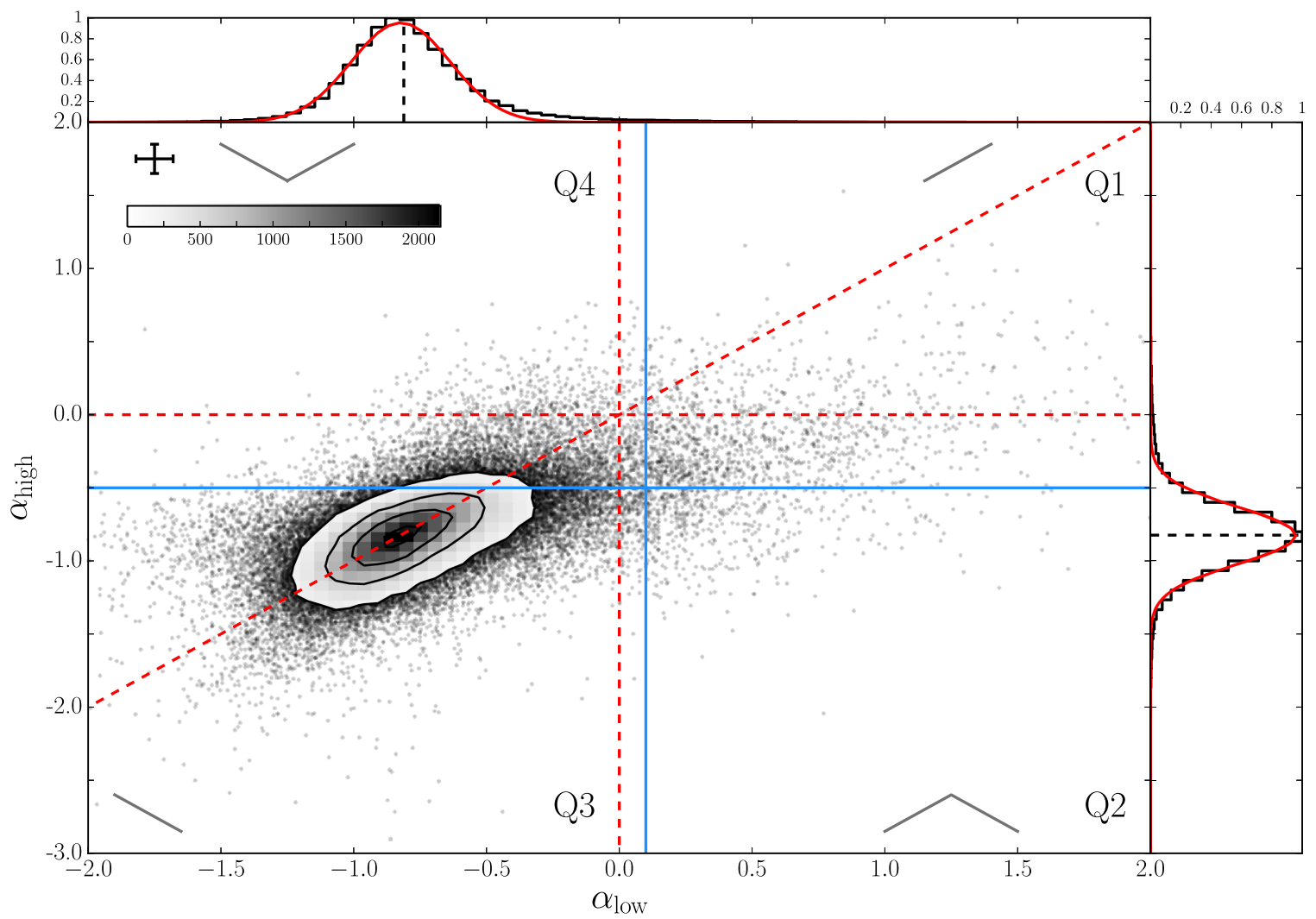

Figure 2. Radio color-color diagram for the 96,628 GLEAM sources that remain after step five of the selection process that is detailed in Table $1 . \alpha_{\text {low }}$ is derived from the 20 GLEAM data points between 72 and $231 \mathrm{MHz}$. $\alpha_{\text {high }}$ was calculated between the GLEAM flux density measurements centered on 189 and 212 MHz and NVSS and/or SUMSS. Contours and a density map are plotted for the region surrounding $\left(\alpha_{\text {low }}, \alpha_{\text {high }}\right) \approx-0.8$ because of the large number of points. The color in the density map conveys the number of sources for each colored pixel, corresponding to the values of the color bar at the top left of the plot. The contour levels represent $100,500,1000$, and 2000 sources, respectively. At $\alpha_{\text {high }}=-0.5$, the horizontal solid blue line delineates the limit below which sources are identified as peakedspectrum sources in the literature. The vertical solid blue line at $\alpha_{\text {low }}=0.1$ marks the separation between the high- and low-frequency peaked-spectrum samples. The dashed red lines represent spectral indices of zero and the one-to-one relation of $\alpha_{\text {low }}$ and $\alpha_{\text {high }}$. Shown in gray at the corners of the plot are mock spectra of the sources for that quadrant. Individual error bars are not plotted to avoid confusion, but the median error bar size for the sample is illustrated at the top left of the figure. The first, second, third, and fourth quadrants discussed in the text are labeled by Q1, Q2, Q3, and Q4, respectively. The two histograms at the top and to the right of the diagram represent the one-dimensional distributions of $\alpha_{\text {low }}$ and $\alpha_{\text {high }}$, normalized by the maximum value of the distribution. The median value and standard deviation of $\alpha_{\text {low }}$ and $\alpha_{\text {high }}$ are $-0.81 \pm 0.19$ and $-0.82 \pm 0.21$, respectively. The median values of $\alpha_{\text {low }}$ and $\alpha_{\text {high }}$ are shown by dashed black lines. Overplotted in red on the histograms are Gaussian fits to the distributions.

index $\alpha_{\text {high }}$ was derived by fitting a power law to the SUMSS and/or NVSS flux density point(s) and to the two GLEAM sub-band flux densities centered on 189 and $212 \mathrm{MHz}$. These two GLEAM sub-band frequencies were chosen because they are near the top of the overall GLEAM band and are from two different GLEAM observing bands. Since systematic fluctuations introduced from the data reduction procedure are largely based on the observing band (see e.g., Figure 18 of HurleyWalker et al. 2017), the selection of two sub-band frequencies from different observing bands minimizes the effect of any systematic and statistical variations on calculating $\alpha_{\text {high }}$.

The radio color-color phase space from 72 to $843 \mathrm{MHz} /$ $1.4 \mathrm{GHz}$ for 96,628 sources is presented in Figure 2 and represents the most densely populated radio color-color plot produced to date. As expected from previous spectral index studies at these frequencies (Lane et al. 2014; Mahony et al. 2016; Hurley-Walker et al. 2017), $\approx 70 \%$ of sources cluster around $\left(\alpha_{\text {low }}, \alpha_{\text {high }}\right) \approx-0.8 \pm 0.2$, which is located in the third quadrant of the diagram, as represented by the symbol Q3 in Figure 2, corresponding to spectra described by an optically thin synchrotron power law. The sources in the first quadrant have a positive spectral index that extends from 72 to $843 \mathrm{MHz} /$ $1.4 \mathrm{GHz}$ and are likely dominated by GPS or HFP sources that are peaking near or above $1 \mathrm{GHz}$. The fourth quadrant is occupied by sources that exhibit convex spectra, which are likely composite sources with a steep-spectrum power-law component at low frequencies and an inverted component at high frequency, which could indicate multiple epochs of AGN activity.

Sources that display concave spectra near the top or above the GLEAM band are located in the second quadrant of Figure 2, and thus it is possible to select peaked-spectrum sources from this region. We note that in the literature, GPS, CSS, and HFP have been defined as having $\alpha_{\text {high }} \leqslant-0.5$ (O'Dea 1998). This definition is also applied to isolate peaked-spectrum sources in this study to facilitate comparison to literature samples, but the continuous distribution in $\alpha_{\text {high }}$ across $\alpha_{\text {high }}=-0.5$ in Figure 2 suggests that this definition is arbitrary. We have included Figure 3 to help guide the eye in identifying the different areas of Figure 2 that we used to isolate peaked-spectrum sources, as outlined in the next step of the selection process.

6a. Sources in the second quadrant of Figure 2 have a turnover in their spectra somewhere between $\approx 200 \mathrm{MHz}$ and $843 \mathrm{MHz} / 1.4 \mathrm{GHz}$. Since the original definition of GPS, CSS, and HFP sources requires $\alpha_{\text {high }} \leqslant-0.5$ (O'Dea 1998), which is shown by the solid blue line in 


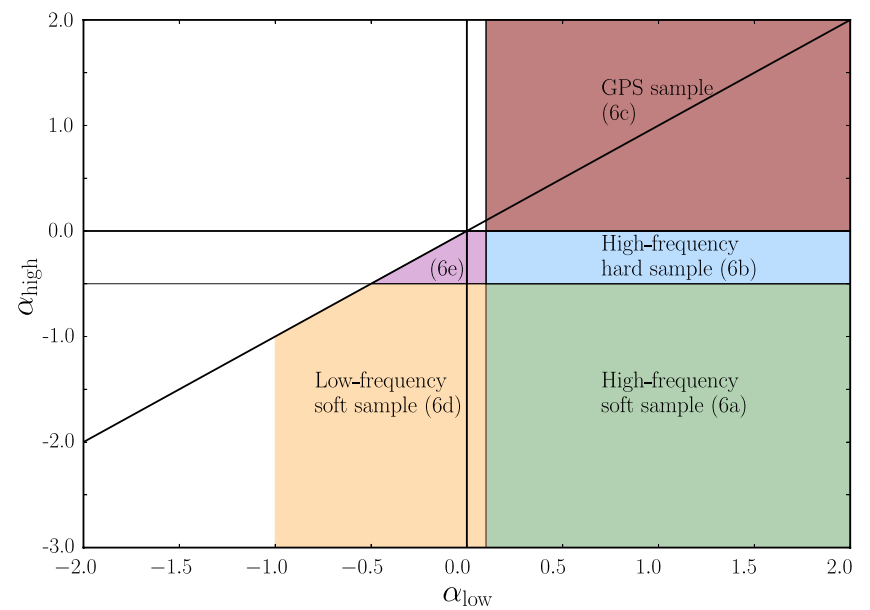

Figure 3. A schematic of the radio color-color plot of Figure 2. The colored areas represent the regions in which peaked-spectrum sources were selected for the different samples outlined in step 6 of the selection process. The green, blue, yellow, purple, and maroon sections are the areas in which the highfrequency soft (step 6a), high-frequency hard (step 6b), GPS (step 6c), lowfrequency soft (step 6d), and low-frequency hard (step 6e) samples were selected, respectively. Note that high frequency here implies that the peak of the source generally occurs above frequencies of $\approx 180 \mathrm{MHz}$. The areas highlighted for the low-frequency samples are only indicative, and $\approx 5 \%$ of the sources outside these regions are located above the one-to-one line.

Figure 2, we use this limit to select peaked-spectrum sources with $\alpha_{\text {high }} \leqslant-0.5$. We also require $\alpha_{\text {low }} \geqslant 0.1$ instead of $\alpha_{\text {low }} \geqslant 0$ because it is significantly more reliable in selecting peaked-spectrum sources as the cut at $\alpha_{\text {low }} \geqslant 0.1$ minimizes the contamination of flat spectrum sources, and because the median uncertainty in $\alpha_{\text {low }}$ is $\approx 0.1$. Sources with $\alpha_{\text {low }}<0.1$ are discussed below. This sample is referred to as the high-frequency soft sample and contains 207 sources.

6b. It is possible for peaked-spectrum sources to exist in the second quadrant above the limit of $\alpha_{\text {high }}=-0.5$. Such sources have wider spectral widths, or higher frequency peaks, than the peaked-spectrum sources identified in step 6a. Therefore we also select peaked-spectrum sources with $\alpha_{\text {low }} \geqslant 0.1$ and $-0.5<\alpha_{\text {high }} \leqslant 0$, and refer to this collection of sources as the high-frequency hard sample. Such a sample may be more contaminated by variable flat-spectrum sources than the soft sample because of the shallower dependence on $\alpha_{\text {high }}$. There are a total of 506 sources in this high-frequency hard sample.

6c. Sources located in quadrant 1 of Figure 2 could be GPS, HFP, and CSS sources that peak above $843 \mathrm{MHz} /$ $1.4 \mathrm{GHz}$. Sources with $\alpha_{\text {low }} \geqslant 0.1$ and $\alpha_{\text {high }}>0$ are therefore also isolated. This sample is generally referred to as the GPS sample, and it contains 261 sources. As a spectral peak in these sources is not directly detected, they are isolated to largely provide spectral coverage below the turnover for known GPS sources.

Since the spectra of the sources are being fit across a large fractional bandwidth to calculate $\alpha_{\text {low }}$, any spectral index derived from a power-law model fit can be artificially flattened if a source displays spectral curvature within the GLEAM band. This means that sources that display a peak between 72 and $231 \mathrm{MHz}$ are shifted toward the third quadrant of Figure 2 and can be calculated to have a negative $\alpha_{\text {low }}$ if the curvature is significant. An example of a negative $\alpha_{\text {low }}$ being derived for a

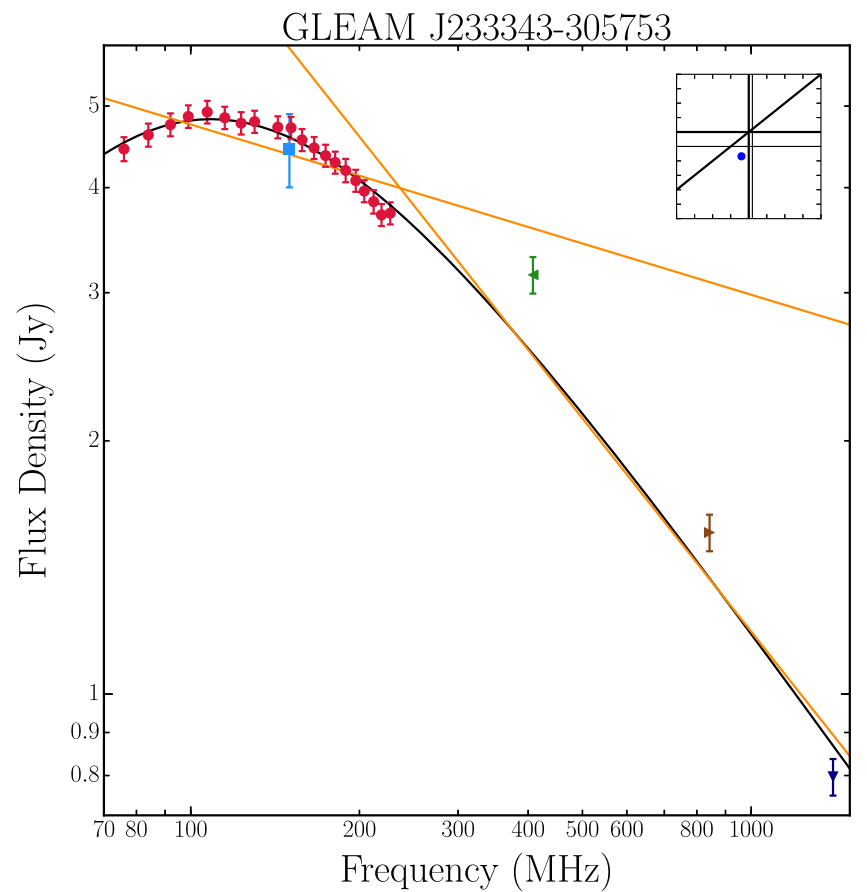

Figure 4. Spectral energy distribution of GLEAM J233343-305753 from $72 \mathrm{MHz}$ to $1.4 \mathrm{GHz}$. The red circles, blue square, green leftward-pointing triangle, brown rightward-pointing triangle, and navy downward-pointing triangle represent data points from GLEAM, TGSS-ADR1, MRC, SUMSS, and NVSS, respectively. The plot inset in the top right corner is the color-color diagram of Figure 2, with the position of GLEAM J233343-305753 marked by a blue circle. Evidently, $\alpha_{\text {low }}$ was calculated to be negative for this source because of the curvature in the GLEAM data. The fit of the generic curved spectral model of Equation (3) to only the GLEAM, SUMSS, and NVSS flux density points is shown by the black curve. The orange lines represent the power-law fits from which $\alpha_{\text {high }}$ and $\alpha_{\text {low }}$ have been derived.

source with significant curvature in the GLEAM band, such that the source is not located in the second quadrant of Figure 2, is provided in Figure 4.

The curvature model described by Equation (2) was also fit to the GLEAM data to test for any evidence of spectral curvature between 72 and $231 \mathrm{MHz}$. Approximately $80 \%$ of sources remaining at step 5 of the selection process show zero or negligible curvature in their spectra covered by the GLEAM band, with only 20,322 sources having a reliable value of the curvature parameter $|q| \geqslant 0.2$, which Duffy \& Blundell (2012) class as significant curvature. The distribution of $q$ against $\alpha_{\text {low }}$ is presented in Figure 5 for sources with $\Delta q \leqslant 0.2$, where $\Delta q$ is the uncertainty in $q$. The requirement of $\Delta q \leqslant 0.2$ ensures that the measurement of $q$ is reliable and is equivalent to the $\mathrm{S} / \mathrm{N}$ cut made in step 3 .

Owing to the effect that curvature has on calculating $\alpha_{\text {low }}$, selecting peaked-spectrum sources in radio color-color phase space is unreliable for sources that display a peak in the GLEAM band. Hence, a source that had $\alpha_{\text {low }}<0.1$, which was the limit in $\alpha_{\text {low }}$ used in radio color-color phase space to select peaked-spectrum sources, was also classified as a peaked-spectrum source if a turnover was identified in the GLEAM band.

The information used to assess a spectral peak in a radio band is set by the $\mathrm{S} / \mathrm{N}$ of the bandwidth available, with the maximum amount of information to detect a turnover transpiring when the peak occurs in the middle of the band. As a peak shifts to the edge of the GLEAM band, the information to reliably detect it declines and is dictated by the 


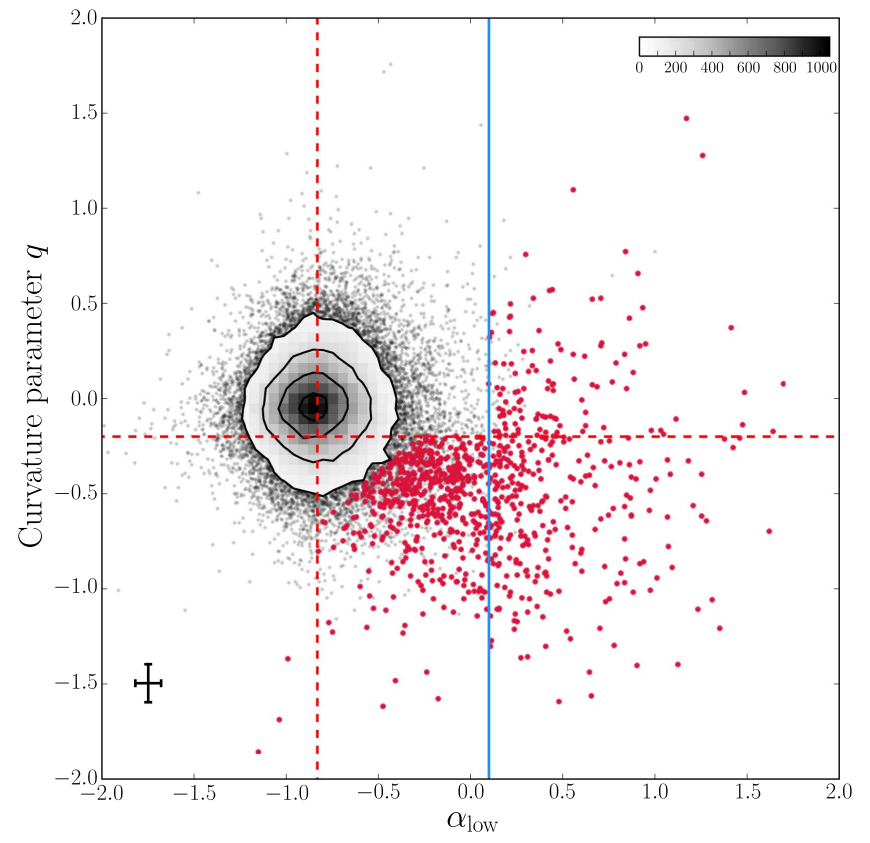

Figure 5. The distribution of the curvature parameter $q$ against $\alpha_{\text {low }}$ for the 96,628 sources remaining after step 5 of the selection process. Only sources with $\Delta q \leqslant 0.3$ are plotted. The vertical and horizontal red dashed lines represent the median of $\alpha_{\text {low }}$ and the cutoff used to indicate significant concave curvature $q=-0.2$, respectively. The color of the density map corresponds to the number of sources in each pixel, as set by the color bar in the top right-hand corner. The contour levels are at 50, 150, 450, and 850 sources. Peak-spectrum sources that are selected in step 6 are plotted in red, with sources above the blue line $\alpha_{\text {low }}=0.1$ mostly identified based on their power-law spectral properties. Sources below this line were selected based on a spectral peak in the GLEAM band. The rough diagonal edge of the peaked-spectrum source distribution around $\alpha_{\text {low }} \approx-0.5$ and $q \approx-0.5$ is due to the requirement of more significant curvature in the GLEAM band to select lower $\mathrm{S} / \mathrm{N}$ sources. The median uncertainties for $q$ and $\alpha_{\text {low }}$ are shown at the bottom left corner of plot.

lever arm closest to the edge of the band. For example, a source that peaks at the central GLEAM frequency of $151 \mathrm{MHz}$ has 10 spectral data points to assess whether the peak is real, while a source that peaks at 85 or $220 \mathrm{MHz}$ only has 2 data points to make the same assessment. In particular, the reliability in identifying a peak at the edge of the band is significantly affected by statistical fluctuations.

If the spectral peak of a source is therefore measured at data point $N_{\mathrm{p}}$, which is above the central frequency data point $N_{\mathrm{c}}$, then a detection of a spectral turnover is considered reliable if

$$
\Delta q \leqslant 0.2-\frac{\left(\sum_{i=N_{\mathrm{p}}}^{N_{\mathrm{H}}} \sigma_{i}^{2}\right)^{1 / 2}}{\sum_{i=N_{\mathrm{p}}}^{N_{\mathrm{H}}} S_{i}},
$$

where $\sigma_{i}$ and $S_{i}$ are the local rms noise and flux density in each sub-band $i$, respectively. $N_{\mathrm{H}}$ represents the highest frequency data point in the band. If $N_{\mathrm{p}} \leqslant N_{\mathrm{c}}$, then the inverse of the sum of the previous equation occurs,

$$
\Delta q \leqslant 0.2-\frac{\left(\sum_{i=N_{\mathrm{L}}}^{N_{\mathrm{p}}} \sigma_{i}^{2}\right)^{1 / 2}}{\sum_{i=N_{\mathrm{L}}}^{N_{\mathrm{p}}} S_{i}},
$$

where $N_{\mathrm{L}}$ is the lowest frequency data point in the band. The second terms of Equations 5(a) and (b) represent the combination of the $\mathrm{S} / \mathrm{Ns}$ in each of the sub-bands above or

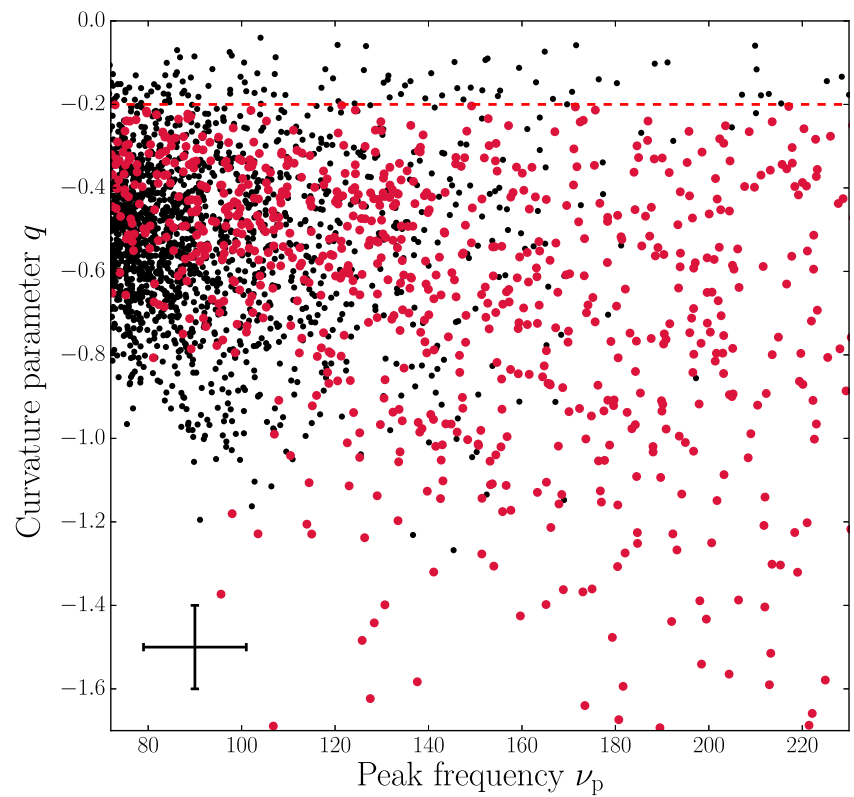

Figure 6. The distribution of the curvature parameter $q$ against the frequency of the peak in the GLEAM band $\nu_{\mathrm{p}}$ for the 96,628 sources remaining after step 5 of the selection process. All sources with $\Delta q \leqslant 0.3$ are plotted in black to provide an indication of the effect the noise has on selecting peaked-spectrum sources. Peaked-spectrum sources selected in step 6 are overplotted in red, with all the sources with $\nu_{\mathrm{p}}$ below $\approx 180 \mathrm{MHz}$ selected on the basis of a peak in the GLEAM band. The concentration of black points toward low $\nu_{\mathrm{p}}$ is due to noise within the GLEAM band. Sources above $\approx 180 \mathrm{MHz}$ are largely identified as peaked-spectrum from their position in radio color-color phase space. The horizontal red dashed line corresponds to limit of $q$ below which curvature in the GLEAM band was considered significant. The median uncertainties for $q$ and $\nu_{\mathrm{p}}$ are plotted at the bottom left corner of diagram.

below the spectral peak. The magnitude of these terms decreases as the number of points available to assess the reliability of a turnover increases. We chose to subtract these terms from 0.2 as the number of false detections of spectral peaks at $151 \mathrm{MHz}$ is below $1 \%$ when $\Delta q=0.2$. This is equivalent to a $\mathrm{S} / \mathrm{N}$ cut of 30 in the wideband image. We note that the functional form of Equations 5(a) and (b) assumes that the noise between the sub-bands is independent. While this is not the case for the GLEAM data at low frequencies due to confusion, since we only test high $\mathrm{S} / \mathrm{N}$ sources for a spectral peak, the effect of confusion is minimized on the spectrum and the noise can be approximated as Gaussian and independent (Franzen et al. 2016; Hurley-Walker et al. 2017).

The distribution of the curvature parameter, $q$, against the frequency of the peak in the GLEAM band, $\nu_{\mathrm{p}}$, for the sources remaining after step 5 of the selection process is presented in Figure 6. The accumulation of black data points toward low $q$ and $\nu_{\mathrm{p}}$ is a function of noise, particularly in the highest subband in GLEAM. A concave spectrum is considered significant if $q \leqslant-0.2$ (Duffy \& Blundell 2012).

Therefore we select peaked-spectrum sources based on the detection of a peak in the GLEAM band, and also separate into soft and hard samples depending on their value of $\alpha_{\text {high }}$ :

$6 \mathrm{~d}$. A source is added to the low-frequency soft sample if it is found with $\alpha_{\text {low }}<0.1, \quad \alpha_{\text {high }} \leqslant-0.5, \quad 72 \mathrm{MHz} \leqslant \nu_{\mathrm{p}}$ $\leqslant 231 \mathrm{MHz}, q \leqslant-0.2$, and $\Delta q$ is less than that set by Equations 5(a) and (b). Such a sample has a size of 394.

6e. For sources to be added to the low-frequency hard sample, it is required that $\alpha_{\text {low }}<0.1,-0.5<\alpha_{\text {high }} \leqslant 0$, 
$72 \mathrm{MHz} \leqslant \nu_{\mathrm{p}} \leqslant 231 \mathrm{MHz}, q \leqslant-0.2$, and $\Delta q$ is less than that set by Equations 5(a) and (b). Similar to the high-frequency hard sample selected in step $6 \mathrm{~b}$, this sample is more likely to contain variable flat-spectrum sources. There are a total of 115 sources in this sample.

The hard and soft samples selected in this way are together referred to as the low-frequency peaked-spectrum sample to differentiate from the samples selected solely on radio colorcolor phase space, which are referred to as the high-frequency peaked-spectrum sample. The locations of more than $95 \%$ of the low-frequency peaked-spectrum samples in radio colorcolor phase space are also displayed in Figure 3.

The selection process also identified two known pulsars as peaked-spectrum sources, PSR J0630-2834 and PSR J16450317 (Manchester et al. 2005). These two sources were removed from the peaked-spectrum samples, and we expect the contamination of pulsars in the total peaked-spectrum sample to be lower than $1 \%$ based on the total number of pulsars detected by the GLEAM survey (Bell et al. 2016).

In this way, we selected a total of 1483 extragalactic peakedspectrum candidates from 96,628 sources. We note that all of the peaked-spectrum candidates were classed as isolated by the cross-matching routine, as detailed in Section 3. The spectra of a source from each of the samples discussed in step 6 of the selection process are presented in Figure 7. The tables providing the characteristics for the high- and low-frequency peaked-spectrum samples are available online in the style of the table presented in Appendix A, as is the GPS sample. The spectra for all peaked-spectrum sources selected are available online as detailed in Appendix B.

\subsection{Sources Peaking Below $72 \mathrm{MHz}$}

It is also possible to identify sources that peak below $72 \mathrm{MHz}$ on the basis of significant curvature in high $\mathrm{S} / \mathrm{N}$ spectra. An example of a source that is beginning to turn over, but peaks below the lowest GLEAM frequency, is shown in Figure 8. However, since we do not detect a peak in the spectrum, these sources are not used in any of the following analysis, but are presented to encourage observations below $72 \mathrm{MHz}$ to confirm the spectral turnover. We identify 36 sources with $q<-0.2$, a turnover below $72 \mathrm{MHz}$, and a S/N greater than 100 in the GLEAM wideband image. We apply such a high $\mathrm{S} / \mathrm{N}$ requirement to produce a reliable collection of sources that peak below $72 \mathrm{MHz}$, rather than a complete sample. The properties of these sources are also provided online in the same format as the table presented in Appendix A.

\subsection{Flux Density Variability and Blazar Contamination}

The reliability of proceeding studies in identifying peakedspectrum sources using multiepoch survey data were significantly affected by radio source variability (e.g., Dallacasa et al. 2000; Snellen et al. 2002). Previously, the often single low-frequency data point used to justify a spectral peak was provided by an observation of the source when it was in a less active phase compared to when high-frequency data were taken. This means that variable sources in previous multiepoch studies could masquerade as peaked-spectrum sources even though the intrinsic spectrum of the source was flat (Tinti et al. 2005). Since GLEAM surveyed the sky in four observing bands, with a two-minute cadence between observing bands, our estimate of the spectral peak and the slope below a turnover is not affected by variability. While high-frequency studies have observed sources such as blazars varying on timescales of hours to months, the radio sky below $1 \mathrm{GHz}$ has been shown to be significantly less variable (McGilchrist \& Riley 1990; Lazio et al. 2010; Bell et al. 2014; Rowlinson et al. 2016).

It is possible that variability affects our determination of the slope above the turnover since data from SUMSS and NVSS were used. However, one strength of having such a wellsampled low-frequency survey is that the defining feature of a peaked-spectrum source, whether the source has peak or a positive spectral slope, is characterized completely by the lowfrequency data. Any high-frequency variability will therefore only move the source between the hard and soft samples, ensuring the source is still identified as a peaked-spectrum source.

As mentioned above, blazars are known contaminants in peaked-spectrum selections because of their variability. While our identification of a peaked-spectrum source may not be significantly affected by variability, the sample will contain some blazars. This is because a peak can occur in the spectrum of a blazar when it is observed during an AGN flare, such that an SSA component dominates the emission spectrum (Torniainen et al. 2007), or because the beaming angle varies. The two samples most likely to be contaminated with blazars are the hard and GPS samples, since the definition of these two samples includes sources with $\alpha_{\text {high }} \sim 0$. By comparing the reported nature of a source in the literature (e.g., Massaro et al. 2015), we estimate that blazars represent $\approx 10 \%$ of sources in the hard and GPS samples, and $<3 \%$ of sources in the soft samples. Future high-resolution imaging, in particular with LOFAR, and low-frequency multiepoch observations from the MWA Transient Survey (MWATS; M. E. Bell et al. 2017, in preparation) survey and the second year of GLEAM survey data will isolate the jet-dominated sources in the samples we presented.

\subsection{Obtaining Redshifts}

We obtained redshift information for the peaked-spectrum candidates selected in Section 5.2 by cross-matching our sample to previous targeted optical observations of GPS, CSS, and HFP sources (Fanti et al. 1990; O'Dea et al. 1991; de Vries et al. 2007; Labiano et al. 2007; Holt et al. 2008), radio-optical studies that combined the large spectroscopic surveys of the Six-degree Field Galaxy Survey (6dFGS; Jones et al. 2004, 2009) and Sloan Digital Sky Survey (SDSS; York et al. 2000) with NVSS and SUMSS (Mauch \& Sadler 2007; Best \& Heckman 2012), targeted quasar surveys (Hewitt \& Burbidge 1987; Osmer et al. 1994; Drinkwater et al. 1997; Hewett $\&$ Wild 2010), investigations that researched the optical properties of a radio source population that were not specifically GPS, CSS, and HFP sources (McCarthy et al. 1996; Jackson et al. 2002; Burgess \& Hunstead 2006; Healey et al. 2008; Mahony et al. 2011), and the NASA/IPAC Extragalactic Database (NED; Helou et al. 1991). ${ }^{14}$

The positions of the NVSS or SUMSS counterparts were used to make the associations in the other catalogs. The NVSS positions were preferenced when the source had both a NVSS and SUMSS counterpart. An association was accepted when the literature source was within $3^{\prime \prime}$ of the radio position. This simple cross-matching scheme was accurate for association

\footnotetext{
${ }^{14}$ http://ned.ipac.caltech.edu/
} 

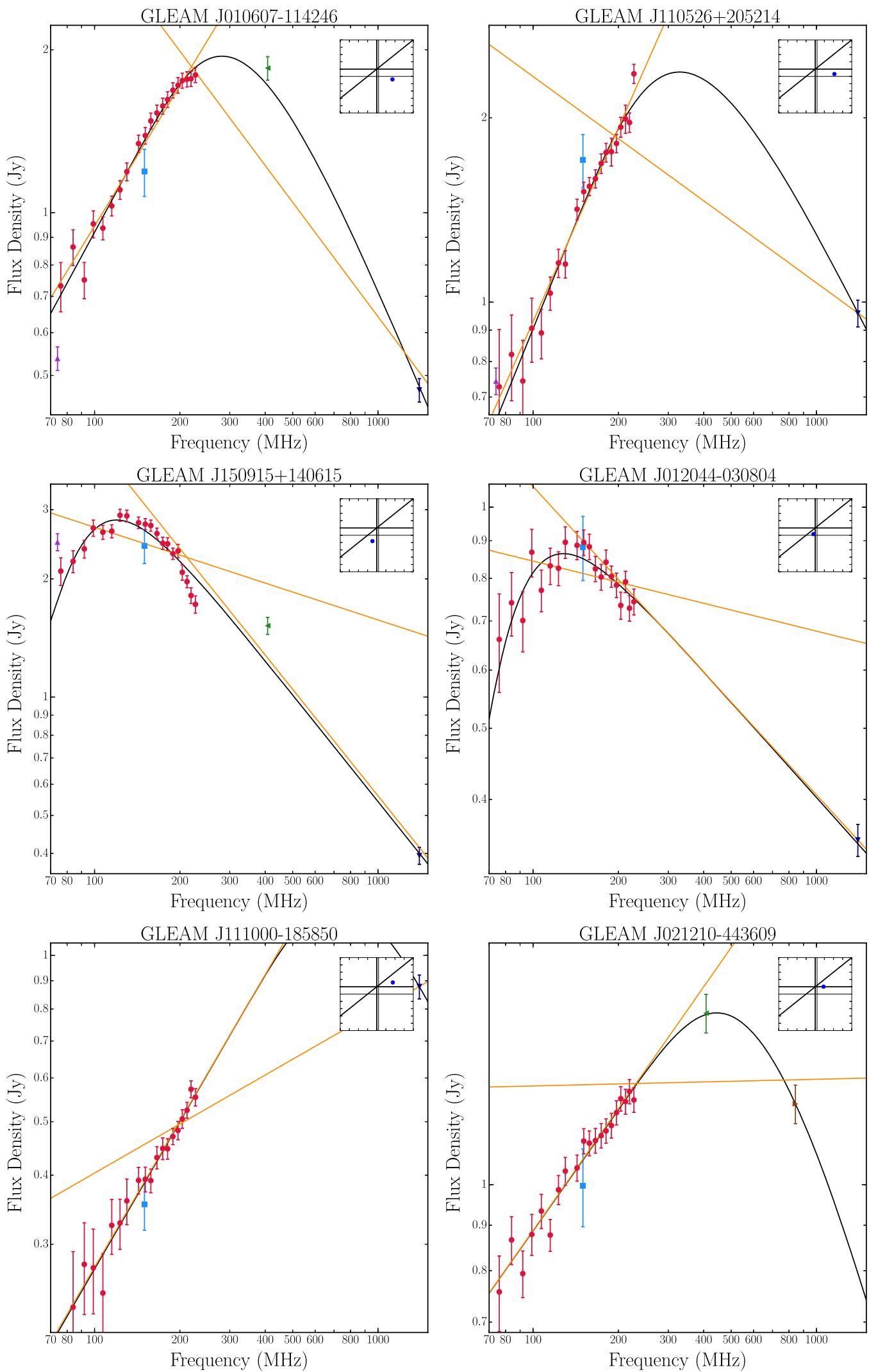

Figure 7. Example spectra for sources drawn from the five different peaked-spectrum samples identified via the selection process. The spectra in the top left, top right, middle left, middle right, and lower left panels are from the high-frequency hard and soft sample, the low-frequency hard and soft sample, and the GPS sample, respectively. The spectrum in the bottom right panel is shown to highlight a source that has $\alpha_{\text {high }} \approx 0$. The purple upward-pointing triangle, red circles, blue square, green leftward-pointing triangle, brown rightward-pointing triangle, and navy downward-pointing triangle represent data points from VLSSr, GLEAM, TGSS-ADR1, MRC, SUMSS, and NVSS, respectively. The fit of the generic curved spectral model of Equation (3) to only the GLEAM and SUMSS and/or NVSS flux density points is shown by the black curve. The power-law fits from which $\alpha_{\text {high }}$ and $\alpha_{\text {low }}$ have been derived are shown in orange. The plot inset in the top right corner displays the position of the source in the color-color diagram of Figure 2 using a blue circle. 


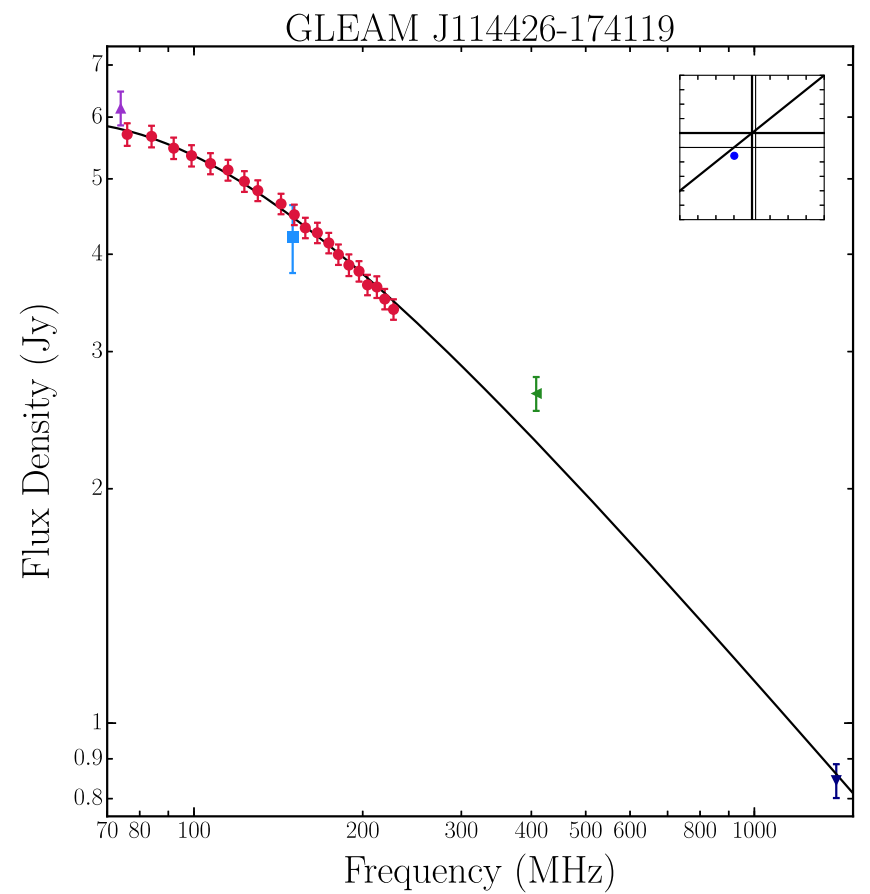

Figure 8. Spectral energy distribution of GLEAM J114426-174119 from $72 \mathrm{MHz}$ to $1.4 \mathrm{GHz}$. The symbols represent data from the same surveys as in Figure 7. While no spectral turnover is detected between $72 \mathrm{MHz}$ and $1.4 \mathrm{GHz}$, the high $\mathrm{S} / \mathrm{N}$ spectrum of GLEAM J114426-174119 allows us to derive $q=-0.31 \pm 0.03$, suggesting that a spectral turnover occurs below $72 \mathrm{MHz}$ The plot inset in the top right corner is the color-color diagram of Figure 2, with the position of GLEAM J114426-174119 marked by a blue circle.

since the proceeding studies have already made an association between the optical and radio sources. We obtain 214 spectroscopic redshifts for the total peaked-spectrum sample, which breaks down into 61, 11, 15, 23, and 104 redshifts in the high-frequency hard sample, high-frequency soft sample, lowfrequency hard sample, low-frequency soft sample, and GPS sample, respectively. The redshift distribution for the peaked-spectrum samples is plotted in Figure 9, which has a median of 0.98 and a highest redshift of 5.19. The literature reference from which the redshift was obtained is detailed in Appendix A.

We note that while NED contains the largest number of redshifts for extragalactic sources of any single database, it has known limitations (see e.g., Hammond et al. 2012). Any redshift information derived from it for a population of sources will be inhomogeneous and incomplete. If multiple spectroscopic measurements of the redshift were reported in NED, the most reliable measurement was selected by inspecting the literature on the source. Additionally, we discarded any photometric redshifts and redshifts that had the " $z$ Quality" field flagged in NED.

\section{Comparison to Known GPS, CSS, and HFP Sources}

To test the reliability of our peaked-spectrum selection criteria, we compared our total peaked-spectrum sample to the known GPS, CSS, and HFP sources from the literature. Only studies that identified GPS, CSS, and HFP sources below a declination of $+30^{\circ}$ were considered. This included the GPS, CSS, and HFP samples identified by Fanti et al. (1990), O'Dea (1998), Stanghellini et al. (1998), Peck \& Taylor (2000), Snellen et al. (2002), Tinti et al. (2005), Labiano et al. (2007),

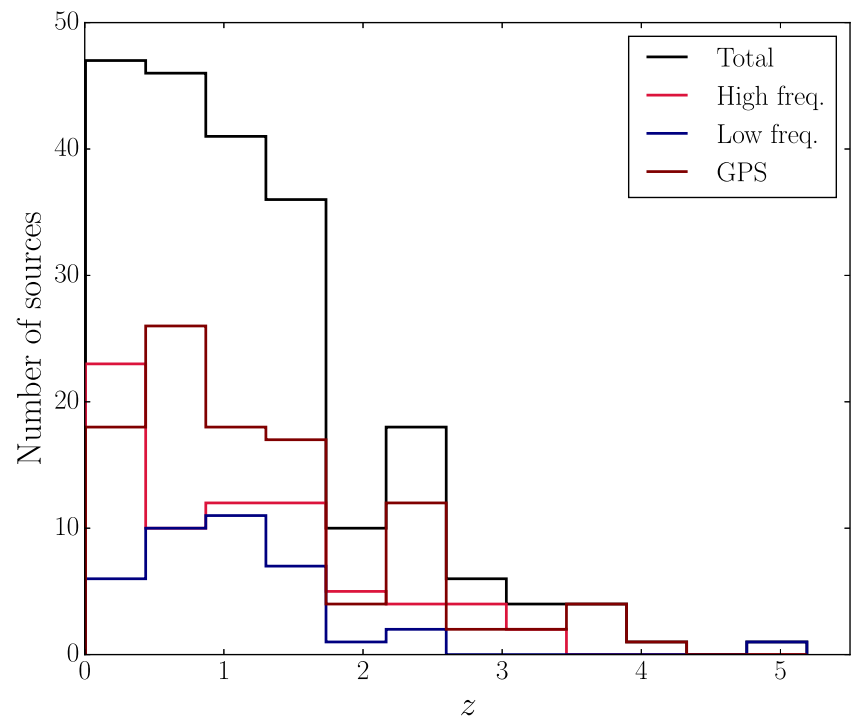

Figure 9. Redshift distribution of the 214 selected peaked-spectrum sources that have reported spectroscopic redshifts. There are 72, 38, and 104 sources with spectroscopic redshifts in the low- and high-frequency peaked-spectrum samples and the GPS sample, respectively. The median of the distribution is 0.98 , with the most distant source at a redshift of 5.19. The total distribution of all identified peaked-spectrum sources is shown in black, while the red, navy, and maroon distributions represent the distribution for the high-frequency sample, the low-frequency sample, and the GPS sample, respectively.

Edwards \& Tingay (2004), and Randall et al. (2011). After removing any duplicates, 157 of the 216 previously known GPS, CSS, and HFP sources that are below a declination of $+30^{\circ}$ have a counterpart in the GLEAM sample from which the peaked-spectrum sources were selected, and 73 of those 157 sources are in our peaked-spectrum samples. The other 59 sources are too faint to be in the GLEAM extragalactic catalog.

The 84 known GPS, CSS, and HFP sources that are not in our total peaked-spectrum sample but are in the GLEAM sample used to isolate the peaked-spectrum sources can be sorted into three spectral categories: 36 sources show no deviation from a power law with a negative slope, 34 sources have flat spectra such that $\alpha_{\text {low }}<0.1$ or $q>-0.2$ in the GLEAM band, and 14 sources display a convex spectrum. We define a convex spectrum as having a negative spectral index in the GLEAM band and a positive spectral index between the end of the GLEAM band and the frequency of SUMSS/NVSS.

Illustrated in Figure 10 are six of the sources that have convex spectra between $72 \mathrm{MHz}$ and $1.4 \mathrm{GHz}$. The convex spectrum category is likely composed of sources that have had multiple epochs of AGN activity, with the peaked-spectrum component above $1 \mathrm{GHz}$ representing recent activity in the core, while the upturn at frequencies below the turnover is suggestive of diffuse, older emission (Baum et al. 1990; Edwards \& Tingay 2004; Torniainen et al. 2007; Hancock et al. 2010). Therefore, the presence of optically thin emission at low frequencies excludes these types of GPS sources from being truly young radio sources. In these types of sources, the peaked component of the spectrum should be interpreted as the radio source being re-started on short timescales, implying the sources have had a long life span but intermittent activity in the nuclear region.

Sources with a peaked component and low-frequency power-law, as shown in Figure 10, have mostly been observed at the center of clusters (Kempner et al. 2004; Hogan 

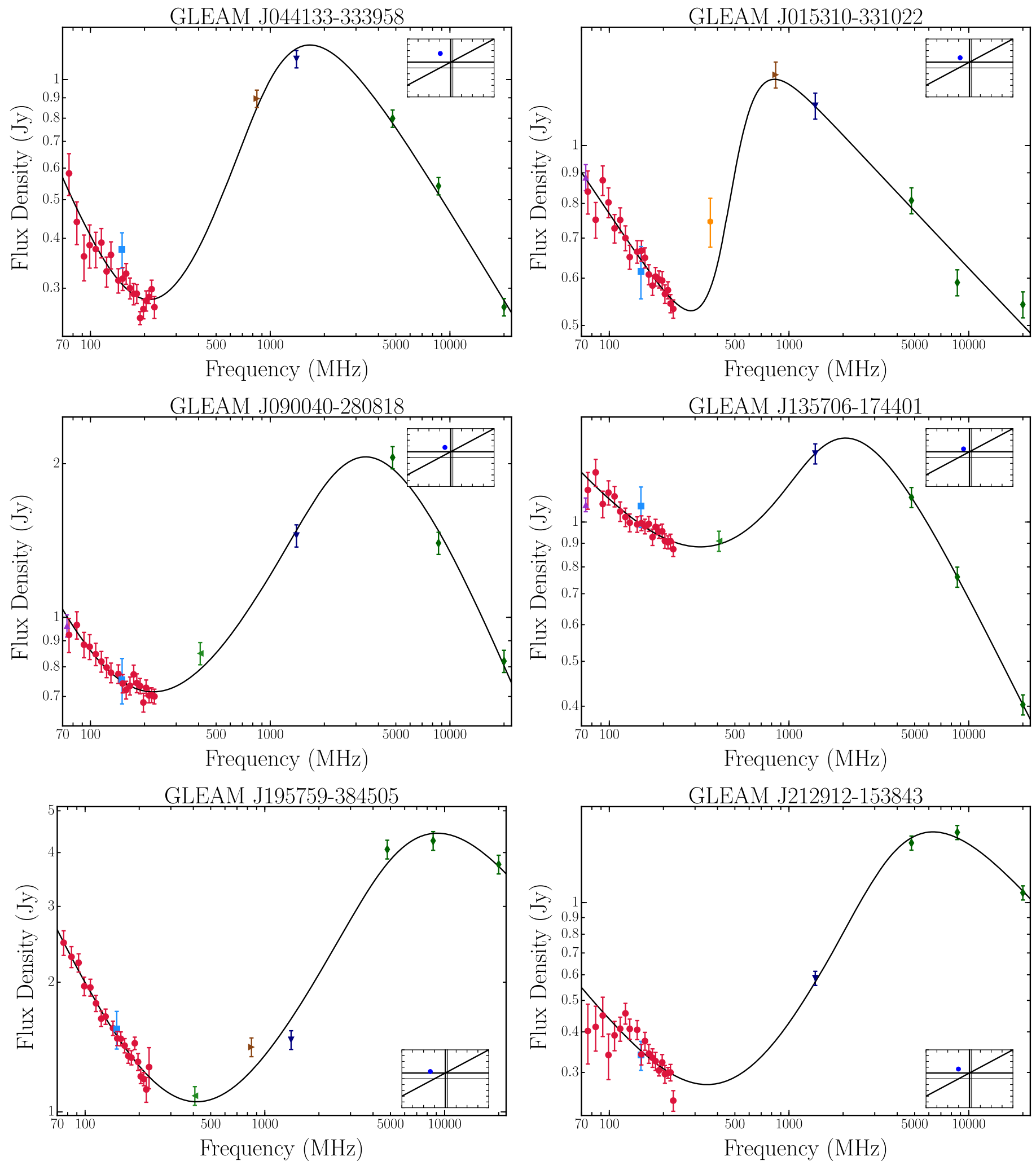

Figure 10. Spectra of six known GPS sources, from $72 \mathrm{MHz}$ to $20 \mathrm{GHz}$, that have a convex pattern between $72 \mathrm{MHz}$ and $1.4 \mathrm{GHz}$. The symbols represent data from the same surveys as in Figure 7, with the dark green diamonds and yellow hexagons depicting data from the AT20G and TXS surveys, respectively. The black curve represents the fit of the generic curved spectral model of Equation (3) with an addition of a low-frequency power law. The blue circle in the inset plot highlights the position of each source in the color-color diagram of Figure 2.

et al. 2015). While the multiepoch activity for sources with this type of spectra is often explained by the high duty cycle expected for AGN hosted by a cool-core cluster (Hogan et al. 2015), only two of our sources are located in cluster environments. For the isolated sources, a varying acceleration rate or the launching of unique knots in the radio jets, which is necessary to produce the observed convex spectra, is likely related to interactions or mergers with nearby galaxies 
(e.g., Hancock et al. 2010; Tadhunter et al. 2012; Brienza et al. 2016).

Since sources with convex spectra could be helpful in understanding the duty cycle of peaked-spectrum sources, we identify 116 convex sources from the GLEAM sample using the criteria $\alpha_{\text {low }}<-0.1$ and $\alpha_{\text {high }}>0.1$. These source are located in the fourth quadrant of Figure 2. Since surveys with frequencies above that of NVSS and SUMSS are required to confirm a turnover in these convex sources, where variability can be significant, we do not use the convex spectrum sources in the further analysis. A list of the convex spectrum sources, presented in a form similar to the form of the table in Appendix A, is available online. We note that high-resolution imaging at low frequencies is needed to measure the size of the low-frequency component and establish the activity timescales.

All of the previously known GPS, CSS, and HFP sources are either in our peaked-spectrum sample or have spectral characteristics that ensure they do not display a spectral turnover between 72 and $843 \mathrm{MHz} / 1.4 \mathrm{GHz}$. This demonstrates that the selection criteria outlined in Section 5 are reliable. It also implies that we have identified 1410 new peaked-spectrum candidates, which is an increase of more than a factor of six in the number of known peaked-spectrum sources below a declination of $+30^{\circ}$ and doubles the number of known peaked-spectrum sources in the entire sky. Additionally, we characterized the peak for the first time for $\approx 95 \%$ of the peaked-spectrum sources we identified.

\section{Comparison to USS Source Samples}

USS sources are defined as compact radio sources with $\alpha<-1$. They have been the focus of searches for highredshift radio AGN (e.g., Jarvis et al. 2001; De Breuck et al. 2006; Klamer et al. 2006; Bryant et al. 2009). Based on the study of the GPS source PKS B0008-421, which was classed as a USS source until observations were conducted below $600 \mathrm{MHz}$, Callingham et al. (2015) hypothesized that the GLEAM survey should find that a portion of the USS population is composed of GPS, CSS, and HFP sources that have ceased nuclear activity and entered a relic phase. The authors argued that PKS B0008-421 had a steep optically thin slope because it had steepened by -0.5 , as expected from the aging of electrons in the continuous injection model of Kardashev (1962), but that the discontinuous transition in the spectrum was not observed because the break frequency had moved to frequencies lower than the spectral turnover. Such a transition could help explain why Klamer et al. (2006) did not find any steepening in the spectra of USS sources above $1 \mathrm{GHz}$. Additionally, if the injection of electrons has ceased in a peaked-spectrum source, strong adiabatic cooling should quickly shift the peak frequency outside the gigahertz regime (Orienti \& Dallacasa 2008), ensuring that a USS source identified above $1 \mathrm{GHz}$ would not be classed as a peakedspectrum source without low-frequency observations.

Since the GLEAM survey has observed the sky with a large fractional bandwidth at low frequencies, we can test if known USS samples selected at high frequencies are significantly composed of sources that display spectral turnovers below $300 \mathrm{MHz}$. We cross-matched our peaked-spectrum samples with the southern USS catalogs of De Breuck et al. (2002), who selected USS sources between $325 \mathrm{MHz}$ and $1.4 \mathrm{GHz}$, and Broderick et al. (2007), who selected sources between 408 and $843 \mathrm{MHz}$. We find that 8 and 5 sources from De Breuck et al.

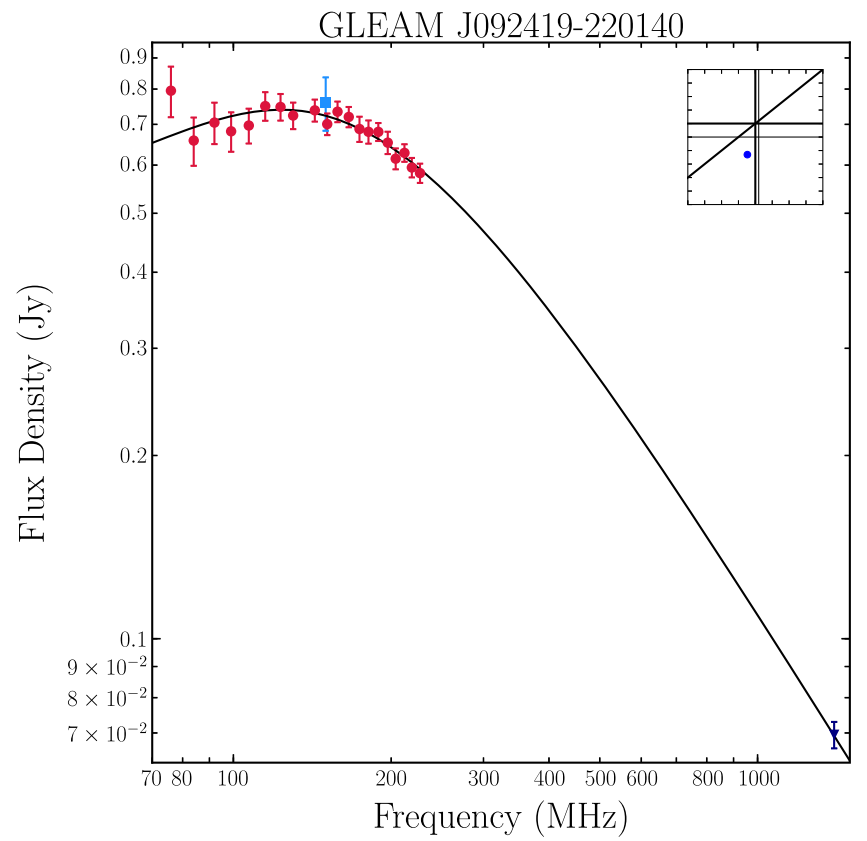

Figure 11. Spectrum of the USS source TN J0924-2201, which is located at $z=5.19$ (van Breugel et al. 1999). The symbols represent the data from the same surveys as detailed in Figure 7. The spectral turnover occurs at $\nu_{\mathrm{p}}=160 \pm 30 \mathrm{MHz}$.

(2002) and Broderick et al. (2007) show turnovers at or below $300 \mathrm{MHz}$, respectively. In comparison, 113 of the 154 USS sources from De Breuck et al. (2002) and 213 of the 239 USS sources from Broderick et al. (2007) are in the GLEAM sample used to select the peaked-spectrum samples. Of the 13 USS sources in which we detect a spectral peak, 2 have reported spectroscopic redshifts at 2.204 and 5.19 (van Breugel et al. 1999; Bryant et al. 2009). The peaked-spectrum of the USS source TN J0924-2201, located at a redshift of 5.19 (van Breugel et al. 1999), is shown in Figure 11.

Follow-up observations of the USS sources with a spectral turnover, ideally with the wideband backends on the Australia Telescope Compact Array (ATCA) and the VLA, are required to confirm whether these sources display high-frequency exponential breaks that are indicative of sources that have ceased nuclear activity (Jaffe \& Perola 1973; Murgia 2003), as for PKS B0008-421.

Alternatively, if these USS sources with low turnover frequencies are actively fueling their AGN and the "youth" scenario of peaked-spectrum sources is correct, the turnover in their spectra could have shifted to low frequencies because they are located at high redshift (Falcke et al. 2004; Coppejans et al. 2015). The idea that these sources are located at high redshift is supported if the steep optically thin slope is a product of the sources being found in environments similar to the center of local, rich clusters. This is because it is possible that the high environmental densities in the early universe both constrained the evolution of the radio jets (Bicknell et al. 1997) and steepened the optically thin spectrum through first-order Fermi acceleration processes from slowed hot-spot advancement (Athreya \& Kapahi 1998; Klamer et al. 2006). Extremely steep-spectrum radio galaxies and GPS/HFP sources are found to reside at the centers of rich clusters in the local universe, and more than $8.5 \%$ of all radio sources located at the center of cool-core clusters have a peaked spectrum (Reuland et al. 2003; Klamer et al. 2006; Hogan et al. 2015). Therefore both a low- 
Table 2

A Summary of the Median Spectral Properties of the peaked-spectrum Sample Presented in this Study, and of the Samples Identified by O'Dea (1998) and Snellen et al. (1998)

\begin{tabular}{llll}
\hline \hline Parameters & This Study & O'Dea (1998) & $\begin{array}{l}\text { Snellen } \\
\text { et al. (1998) }\end{array}$ \\
\hline $\begin{array}{l}\text { Selection fre- } \\
\quad \text { quency (MHz) }\end{array}$ & $72-1400$ & Inhomogeneous & $325-5000$ \\
$\begin{array}{l}\text { Faintest Spectral Peak } \\
\quad \text { Flux Density (Jy) }\end{array}$ & 0.16 & 0.3 & 0.04 \\
Number of Sources & 1483 & 69 & 47 \\
$\alpha_{\text {thin }}$ & $-0.77_{-0.38}^{+0.31}$ & $-0.75 \pm 0.15$ & $-0.77 \pm 0.15$ \\
$\alpha_{\text {thick }}$ & $0.88_{-0.49}^{+0.71}$ & $0.56 \pm 0.20$ & $0.80 \pm 0.18$ \\
Observed $\nu_{\mathrm{p}}(\mathrm{MHz})$ & $190_{-90}^{+190}$ & $750_{-250}^{+1200}$ & $1500_{-500}^{+700}$ \\
$z$ & 0.98 & 0.84 & 1.01 \\
Intrinsic $\nu_{\mathrm{p}}(\mathrm{MHz})$ & $440_{-250}^{+560}$ & $850_{-100}^{+2500}$ & $2840_{-860}^{+1900}$ \\
$P_{5 \mathrm{GHz}}\left(\log _{10} \mathrm{~W} \mathrm{~Hz}{ }^{-1}\right)$ & $26.5_{-1.2}^{+0.7}$ & $27.6_{-1.0}^{+0.7}$ & $26.2_{-0.4}^{+0.5}$ \\
\hline
\end{tabular}

Note. The spread in the values quoted represents the difference between the median and the 16th or 84 th percentiles in the distributions. The parameters listed for this study are for the total high- and low-frequency peaked-spectrum samples, except for $\alpha_{\text {thick}}$, which is only for the high-frequency peakedspectrum sample. The selection frequency for the peaked-spectrum sample studied by O'Dea (1998) is listed as inhomogeneous because the sample was assembled from different literature sources, such as Stanghellini et al. (1998) and Fanti et al. (1990).

frequency turnover and steep spectral slope above the turnover could be excellent priors in searching for high-redshift galaxies. This is explored in greater detail in Section 8.

While Blundell et al. (1999) posited that the correlation between redshift and steep optically thin spectral indices was a product of radio luminosity and Malmquist bias, adding the requirement of a turnover breaks the degeneracy of the evolution of radio luminosity and different survey flux density limits. Provided that USS sources with low-frequency turnovers are young radio AGN, they therefore represent excellent high-redshift candidates.

\section{Spectral Properties of the Peaked-spectrum Sample}

Since we have derived a unique peaked-spectrum sample by exploiting a wideband low-frequency survey, we have the opportunity of using a new sample to extend on previous work in understanding the physical properties of peaked-spectrum sources. It is also important to compare the spectral properties of this sample with archetypal peaked-spectrum samples, such as those presented by O'Dea (1998) and Snellen et al. (1998), to test whether the spectral properties of known GPS, CSS, and HFP sources are consistent with the newly characterized peaked-spectrum sources, and to understand the biases in our selection method. A summary of the comparison of the total peaked-spectrum sample with the samples studied by O'Dea (1998) and Snellen et al. (1998) is presented in Table 2.

The observed distribution of the peak frequency and flux density for our peaked-spectrum samples, with the different samples identified by their colors, is presented in Figure 12. Clearly, the selection criteria employed are a function of both peak frequency and flux density, with no sources detected with a peak frequency below $72 \mathrm{MHz}$ or above $1.4 \mathrm{GHz}$, or below the flux density cut made at $0.16 \mathrm{Jy}$. Few sources are detected with peak flux densities below $0.3 \mathrm{Jy}$ beneath $100 \mathrm{MHz}$ or above $600 \mathrm{MHz}$ since the detection of a peak at the edge of the

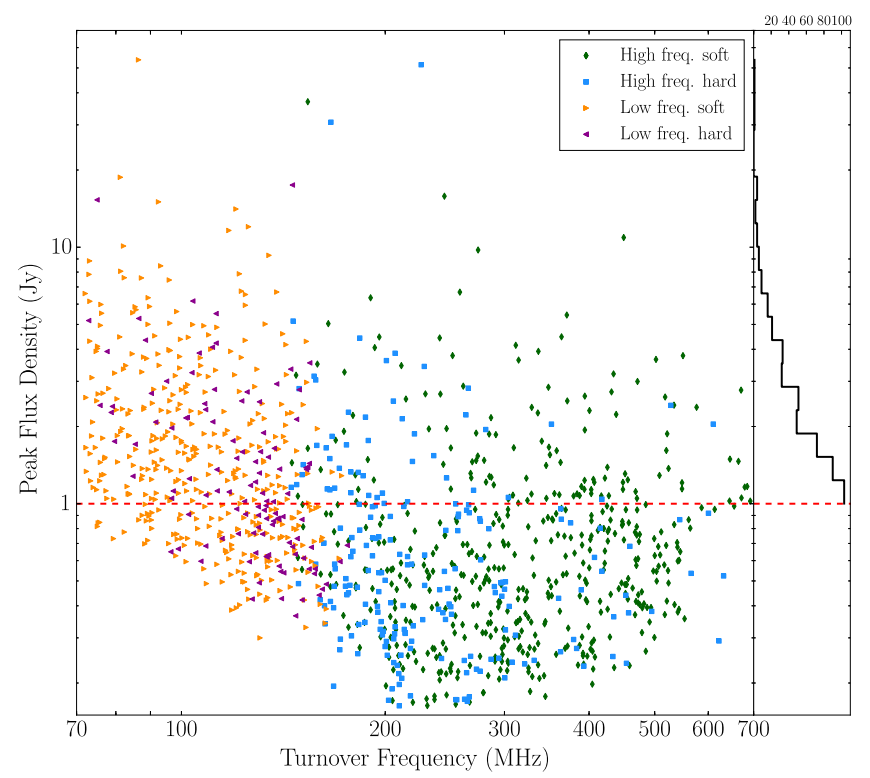

Figure 12. The distribution of the spectral turnover frequency and the peak flux density for the GLEAM sources in which a spectral turnover is detected. The colors represent the different peaked-spectrum samples, as communicated by the legend. The red dashed line represents the peak flux density in which the total peaked-spectrum sample is complete, which corresponds to $\approx 1 \mathrm{Jy}$. The histogram on the right represents the distribution of peak flux densities above $1 \mathrm{Jy}$.

observed band relies on high $\mathrm{S} / \mathrm{N}$ statistics. As expected from the definition of the different samples, the high-frequency soft sample has the highest frequency peaks, with the transition between sources being selected based on radio color-color phase space and a peak in the GLEAM band occurring around $\approx 180 \mathrm{MHz}$. Additionally, the continuity of the distribution of parameters in Figure 12 demonstrates that the cut using $\alpha_{\text {high }}$ to separate the hard and soft population is arbitrary.

As is evident from the distribution of peaked-spectrum sources in Figure 12, the obvious biases introduced by the selection criteria in the flux density and frequency are eliminated above peak flux densities of $1 \mathrm{Jy}$. We therefore infer that the peaked-spectrum samples are reasonably complete above $1 \mathrm{Jy}$, allowing a comparison of the number of peaked-spectrum sources that comprise the GLEAM sample. A total of 505 sources in the high- and low-frequency peakedspectrum samples have a peak flux density at or above $1 \mathrm{Jy}$, compared to 11,400 sources from the sample from which these peaked spectra were drawn. This shows that approximately $4.5 \%$ of the radio population in the GLEAM extragalactic catalog are identified as peaked-spectrum sources. This is significantly less than the canonical $10 \%$ of GPS sources occupying the total number of radio source population for surveys completed around or above $1 \mathrm{GHz}$ (O'Dea 1998), implying that either the number of peaked-spectrum sources declines with decreasing frequencies or that the number of sources that follow a power law increases.

The histogram on the right of Figure 12 demonstrates that the proportion of peaked-spectrum sources to the total radio source population changes slightly with flux density. For example, a 4 Jy peak flux density cut implies that $6.5 \%$ of the radio source population is composed of peaked-spectrum sources. Additionally, the fraction of peaked-spectrum sources will increase with survey frequency due to the different spectral index of two populations selected to be peaked at different 


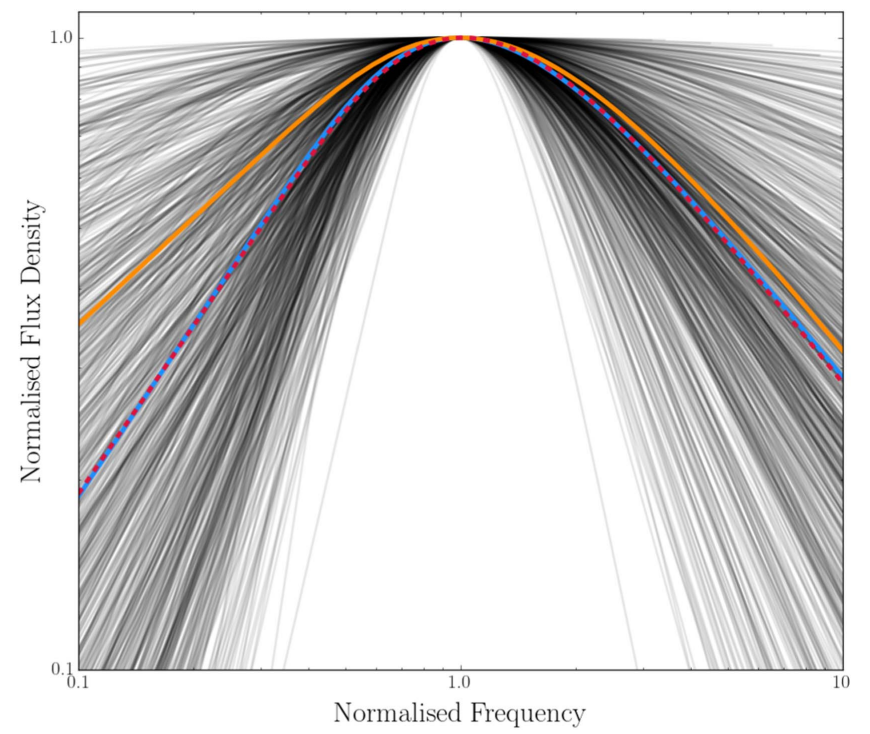

Figure 13. The spectra of the high-frequency soft and hard peaked-spectrum samples, as fit by Equation (3), normalized by the peak flux density and frequency. The dashed red line represents the median spectra for the sample, with median thick and thin spectral indices of $0.88_{-0.49}^{+0.71}$ and $-0.77_{-0.38}^{+0.31}$, respectively. The solid blue and orange lines are the median values of the GPS samples presented by Snellen et al. (1998) and O’Dea (1998), respectively.

frequencies. Such a change in frequency and luminosity is expected from the Snellen et al. (2000) evolutionary model of peaked-spectrum sources. The frequency dependency of the source counts will be the focus of a future study.

To understand the variation in the spectral shape of the selected peaked-spectrum sources, we show the spectra normalized by the peak frequency and flux density, as described by the general curved spectral model of Equation (3), for our high-frequency peaked-spectrum sample in Figure 13. The median spectrum of our high-frequency sample and the GPS and CSS samples presented by Snellen et al. (1998) and O'Dea (1998) are overplotted. The median spectrum of the high-frequency peaked-spectrum sample is almost identical to the spectrum derived by Snellen et al. (1998), but shallower than the median spectrum identified by O'Dea (1998).

The distributions of $\alpha_{\text {thin }}$ and $\alpha_{\text {thick }}$, as derived from fitting Equation (3), are presented in Figure 14. The distributions of $\alpha_{\text {thin }}$ and $\alpha_{\text {thick }}$ are more accurate and useful in understanding the properties of the peaked-spectrum samples than the distribution of $\alpha_{\text {low }}$ and $\alpha_{\text {high }}$ since $\alpha_{\text {thin }}$ and $\alpha_{\text {thick }}$ are not artificially flattened or steepened by the presence of curvature in the spectrum, as evident in the spectra illustrated in Figure 7. We note that only peaked-spectrum sources that had a reduced $\chi^{2}<3$ were used in the following analysis, discarding $\approx 3 \%$ of the sample. Since the spectra in the low-frequency peakedspectrum samples are not completely sampled below the turnover, only the distribution for the total high- frequency sample is presented for $\alpha_{\text {thick. The distribution for the total }}$ peaked-spectrum sample is presented for $\alpha_{\text {thin }}$ because the high-frequency spectral slope is completely sampled for most of the sources. The median value and the range to the 16th and 84th percentiles of the optically thick and thin spectral index distributions for the peaked-spectrum sample are $0.88_{-0.49}^{+0.71}$ and $-0.77_{-0.38}^{+0.31}$, respectively. In comparison, Snellen et al. (1998) and O'Dea (1998) report median values of the optically thick spectral indices of $0.80 \pm 0.18$ and $0.56 \pm 0.20$, and optically thin spectral indices of $-0.75 \pm 0.15$ and $-0.77 \pm 0.15$, respectively. The comparison of these values for the different samples is succinctly presented in Table 2.

The large dispersion in the optically thin and thick spectral indices is a product of our selection process being sensitive to the type of peaked-spectrum sources identified previously and to both a spectrally flatter and steeper population than previous studies. NVSS and SUMSS are over two orders of magnitude more sensitive than the GLEAM survey, and the GLEAM survey observed sources with a wide fractional bandwidth, implying that we can select peaked-spectrum sources that are extremely inverted or relatively flat when compared to the peaked-spectrum sources identified by Snellen et al. (1998) and O'Dea (1998). While Snellen et al. (1998) estimated that their survey missed $\sim 10 \%$ of the peaked-spectrum sources that had steep optically thick spectra $\left(\alpha_{\text {thick }}>1\right)$, the large dispersion of the optically thick spectral index of our high-frequency peakedspectrum samples suggests that the number of sources missed could be be as high as $20 \%$.

In particular, the wide spread in optically thick spectral indices is strongly indicative that the turnover in the spectrum is caused by an inhomogeneous environment that differs from source to source (de Vries et al. 1997), independent of whether the absorption is a product of ionized clouds of plasma acting as a screen or many synchrotron self-absorbed components.

\subsection{Observed Spectral Turnover Frequency Relationships}

The distribution of the observed turnover frequencies for the low- and high-frequency peaked-spectrum samples are presented in the left panel of Figure 15, with the median plotted at $190 \mathrm{MHz}$. The distribution of the observed turnover frequencies is a function of the selection criteria, since a peakedspectrum source in this study is either identified by a spectral peak in the GLEAM band or by two power-law slopes that traced a concave curvature. The transition between the two selection methods is evident by the slight increase in the number of peaked-spectrum sources identified above peak frequencies of $\approx 180 \mathrm{MHz}$, corresponding to the small increase in sensitivity when using radio color-color phase space to assess whether a source is peaked. Somewhat surprisingly, there is a monotonic decline in turnover frequencies between 180 and $500 \mathrm{MHz}$, where the sensitivity of the selection method is uniform. Above $500 \mathrm{MHz}$, the decline in the number of peaked-spectrum sources identified is a function of the decreasing frequency coverage above the turnover. Additionally, the flattening of the number of sources at $\approx 100 \mathrm{MHz}$ is also due to the selection criteria, implying the distribution will likely continue to monotonically increase at frequencies below $100 \mathrm{MHz}$.

To minimize the impact biases introduced by the selection criteria, the distribution of observed turnover frequencies for the sources with peak flux densities greater than $1 \mathrm{Jy}$ is also plotted in red in Figure 15. The rise in the number of sources toward low turnover frequencies is still evident, suggesting a frequency evolution of the sources. It is also possible that the decline of sources toward higher turnover frequencies could be due to the interplay between the number of sources observed as a function of size and physical size being inversely proportional to the turnover frequency in SSA sources. This explanation implies that there are many more unresolved radio sources with linear sizes greater than $\approx 1 \mathrm{kpc}$ than with linear sizes smaller than $\approx 1 \mathrm{kpc}$ at low radio frequencies. The decline of the 

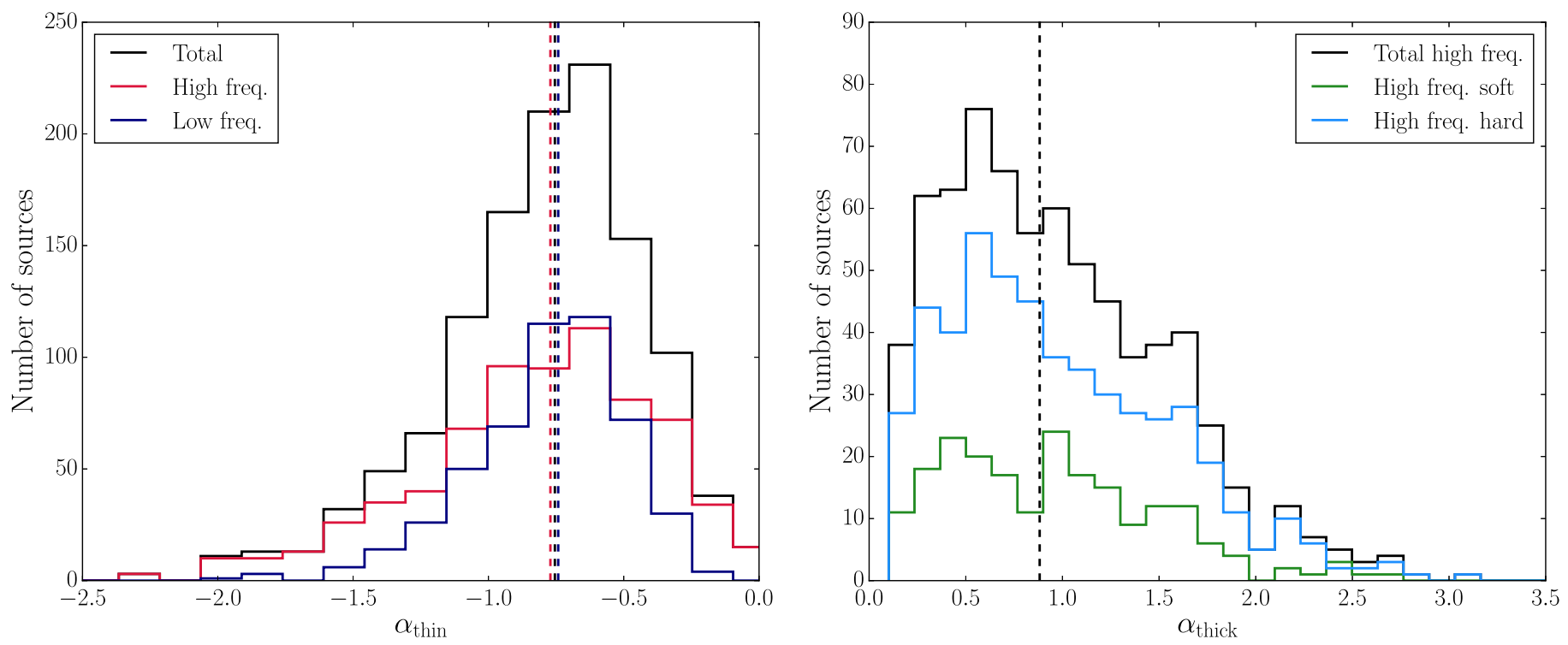

Figure 14. Left panel: the spectral index distribution of $\alpha_{\text {thin }}$. The distribution for the total, high-frequency, and low-frequency peaked-spectrum samples are shown in black, red, and navy, respectively. The median for the total peaked-spectrum sample is plotted as a black dashed line at $\alpha_{\text {thin }}=-0.77$. The medians for the low- and high-frequency sample are presented as red and navy dashed lines, respectively, at approximately the same value as the median for the total sample. Right panel: the spectral index distribution of $\alpha_{\text {thick }}$. The black, green, and blue histograms represent the distribution of $\alpha_{\text {thick }}$ for the total high-frequency, hard high-frequency, and soft high-frequency peaked-spectrum sample, respectively. The dashed black line illustrates the median $\alpha_{\text {thick }}=-0.88$ for the total high-frequency sample.

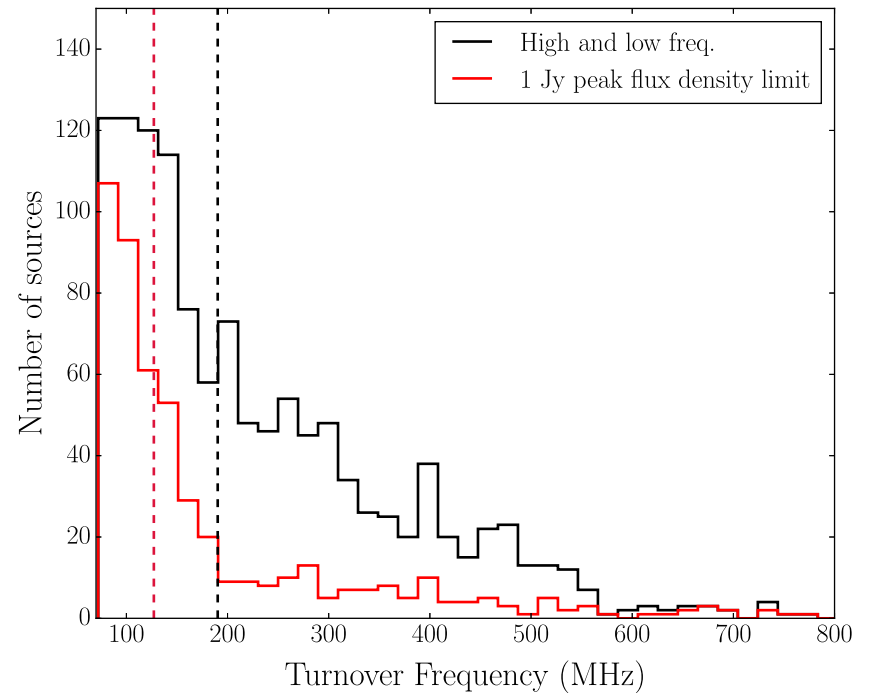

Figure 15. Distributions of the observed spectral turnover frequency for the low- and high-frequency peaked-spectrum samples and for the peakedspectrum sources in those samples with peak flux densities greater than $1 \mathrm{Jy}$, shown in black and red, respectively. The median of the observed turnover frequency for the total low- and high-frequency peaked-spectrum samples is plotted by a dashed black line at $190 \mathrm{MHz}$. The median for the sources with peak flux densities greater than $1 \mathrm{Jy}$ is shown by the dashed red line at $130 \mathrm{MHz}$. The linear bin sizes are $20 \mathrm{MHz}$.

number of peaked-spectrum sources with increasing turnover frequency is discussed in more detail in Section 8.3.

The variation in the distribution of the observed turnover frequencies with the optically thin and thick spectral indices is shown in Figure 16. The concentration of sources at values of $\alpha_{\text {thin }}<-1$ and $\alpha_{\text {thin }} \approx-0.1$ at higher turnover frequencies is also a product of the selection method because either a turnover will be correctly detected at frequencies greater than $500 \mathrm{MHz}$ if the optically thin spectrum is steep (i.e., $\alpha_{\text {thin }}<-1$ ), or the NVSS/SUMSS point is sampling the spectrum near the turnover, causing the calculation of $\alpha_{\text {thin }}$ to be artificially flattened. Additionally, the optically thick spectral index shows no dependence on observed turnover frequency, as evident in the right panel of Figure 16.

\subsection{Intrinsic Spectral Turnover Frequency Relationships}

Since the observed turnover frequency is convolved with source evolution and redshift, we repeat the same analysis presented in the previous section with the 110 sources in the high and low frequency peaked-spectrum samples that have reported spectroscopic redshifts. We find no trend with the observed turnover frequency with redshift, as evident in Figure 17. While de Vries et al. (1997) noted that GPS sources with spectral peaks above $5 \mathrm{GHz}$ could be found preferentially at high redshift, our sample is not sensitive to these high frequency sources. The lack of a trend in turnover frequency with redshift, but with a wide spread in redshift, is consistent with the hypothesis that sources with observed turnover frequencies below $500 \mathrm{MHz}$ are composed of high redshift GPS-like sources and local CSS-like sources (Coppejans et al. 2016). Higher resolution low frequency imaging to measure the linear sizes of the sources is necessary to decouple the low redshift CSS-like population and high redshift GPS-like population. This will be the focus of a follow-up study. Additionally, the dearth of peaked-spectrum sources at redshifts less than 0.1 is consistent with the luminosity function of GPS and CSS sources being flatter than that of large-size radio sources (Snellen et al. 2000; Mauch \& Sadler 2007; Best \& Heckman 2012).

The distribution of the rest frame turnover frequencies for the low- and high-frequency peaked-spectrum samples is presented in Figure 18 and is compared to the distributions presented by O'Dea (1998) and Snellen et al. (1998). We note that the sample presented by O'Dea (1998) is largely composed of the GPS sources studied by Stanghellini et al. (1998) and of the CSS sources identified by Fanti et al. (1990). The peakedspectrum sample has a median rest-frame turnover frequency of $440_{-250}^{+560} \mathrm{MHz}$, compared to $850_{-100}^{+2500}$ and $2840_{-860}^{+1900} \mathrm{MHz}$, for 

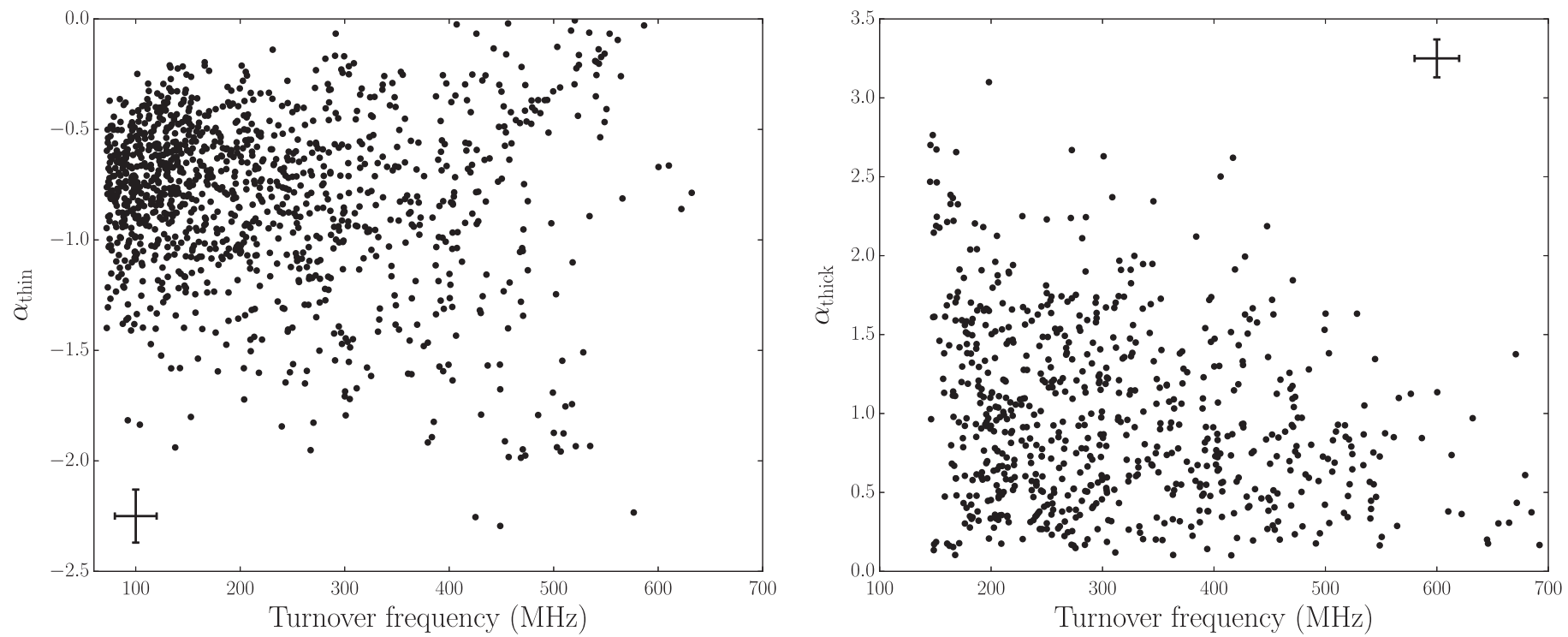

Figure 16. The variation in spectral turnover frequency with $\alpha_{\text {thin }}$ (left panel), for the high- and low-frequency peaked-spectrum source samples, and $\alpha_{\text {thick }}($ right panel), for the high-frequency peaked-spectrum source sample. While there is a trend of higher turnover frequency having steeper $\alpha_{\text {thin }}$, this is a product of the selection method. $\alpha_{\text {thick }}$ has no dependence on the turnover frequency, consistent with $\alpha_{\text {thick }}$ being a product of the individual environments. The median uncertainties of the values are located in the bottom left and top right corner of the plots.

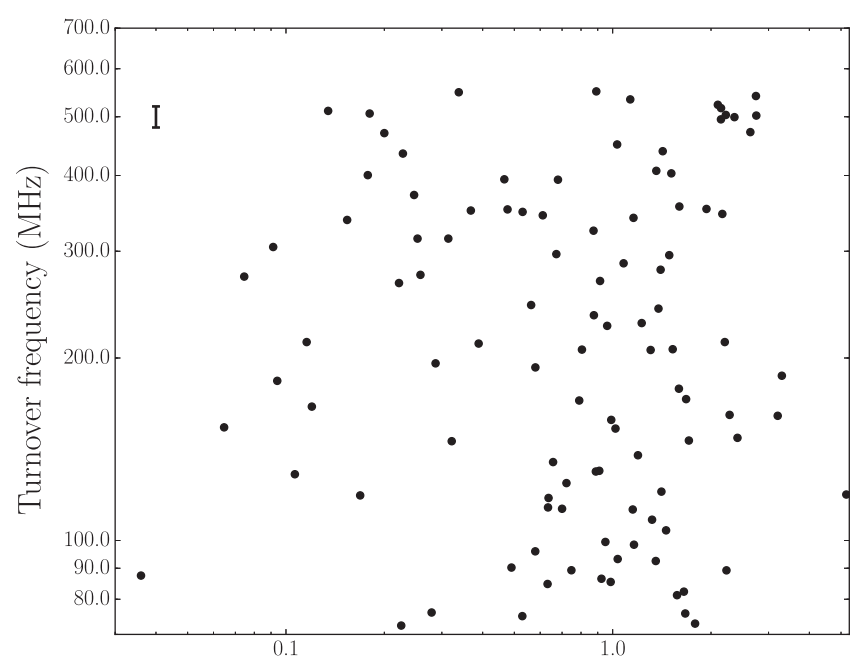

Figure 17. The variation of redshift with the observed frequency of the spectral turnover for the 110 peaked-spectrum sources with reported spectroscopic redshifts and detected spectral turnovers. The random spread is consistent with the sample population being composed of both low-redshift CSS sources and high-redshift GPS sources. The median uncertainty of $20 \mathrm{MHz}$ in the observed turnover frequency is shown in the top left of the plot.

the samples identified by O'Dea (1998) and Snellen et al. (1998), respectively. Our sample has a surplus of sources with intrinsic turnover frequencies between $\approx 150$ and $500 \mathrm{MHz}$, compared to the other two peaked-spectrum samples, consistent with the observing frequencies of the surveys used to compose our sample.

As suggested in Section 7, sources that have an optically thin spectral index $<-1$ and observed turnovers below $300 \mathrm{MHz}$ could be preferentially found at high redshifts. To test this hypothesis, the left panel of Figure 19 shows the variation in $\alpha_{\text {thin }}$ with the observed turnover frequency, colored by the redshift of the source. All sources with a turnover frequency lower than $250 \mathrm{MHz}$ and $\alpha_{\text {thin }}<-1.2$ are located at $z>2$. However, there is significant amount of scatter in the

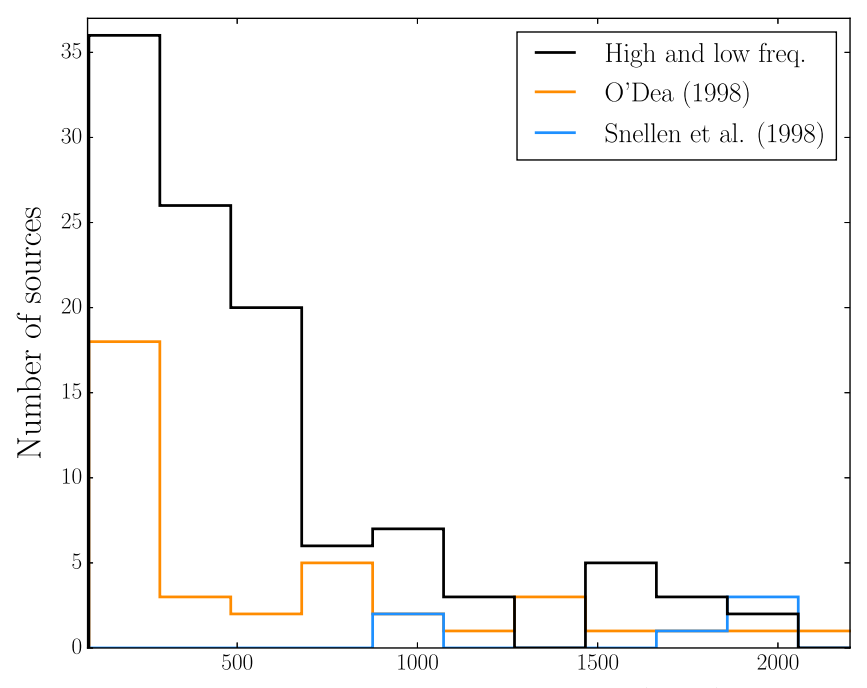

Rest frame turnover frequency $(\mathrm{MHz})$

Figure 18. The distribution of the rest frame turnover frequency for the 110 sources from the low and high frequency peaked-spectrum samples that have reported spectroscopic redshifts, shown in black. The dark orange histogram represents the rest-frame turnover frequency distribution of the GPS and CSS of the O'Dea (1998) sample, which is mostly composed of the GPS and CSS sources identified by Stanghellini et al. (1998) and Fanti et al. (1990), respectively. In particular, the low-frequency bin is dominated by the Fanti et al. (1990) CSS source sample. The blue histogram represents the distribution of the sample of peaked-spectrum sources identified by Snellen et al. (1998). Note that the highest rest-frame turnover frequency for a source in our highand low-frequency peaked-spectrum samples is $\approx 2000 \mathrm{MHz}$, while the restframe turnover frequency distribution of the sources presented by O'Dea (1998) and Snellen et al. (1998) extends beyond $2000 \mathrm{MHz}$.

dependence, and a targeted optical follow-up campaign for the entire peaked-spectrum sample is required to make a conclusive statement. We note that the right panel of Figure 19 shows no dependence of turnover frequency and the optically thick spectral index with redshift, consistent with the spectral turnover being a product of the individual environment of each source. 

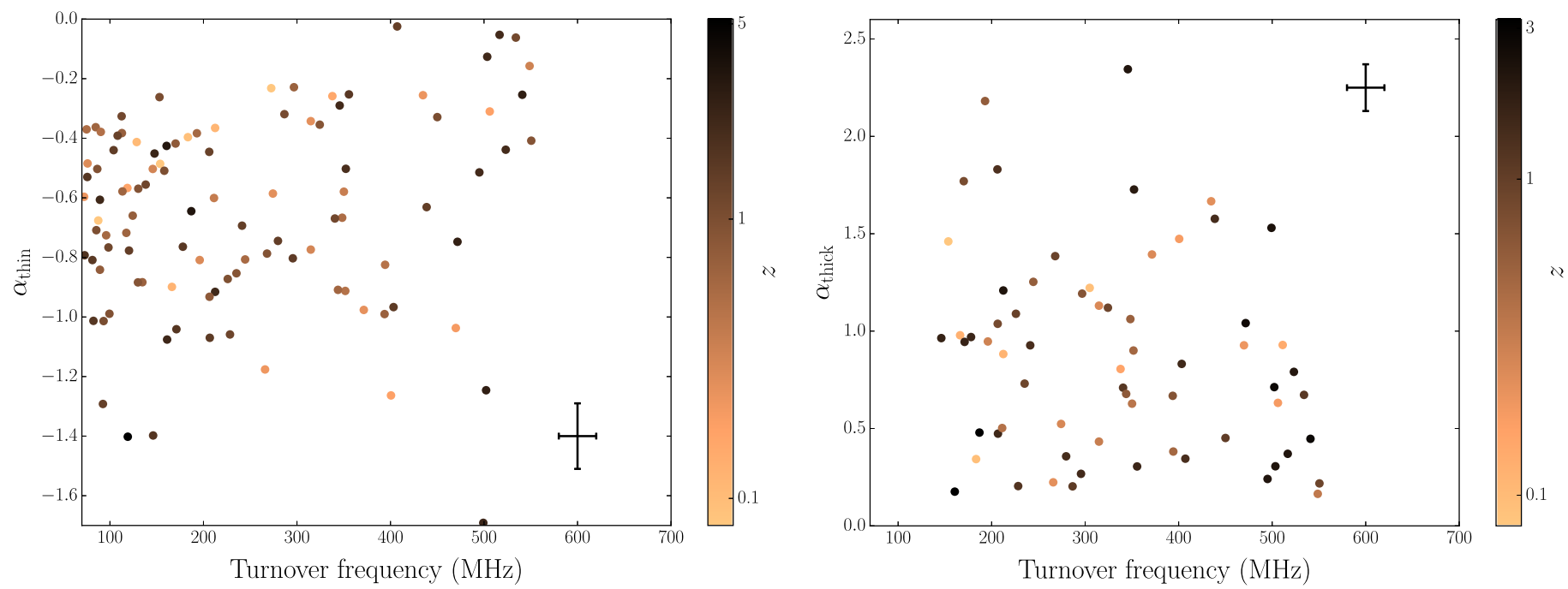

Figure 19. Observed turnover frequency against $\alpha_{\text {thin }}$ (left panel) for both the high- and low-frequency peaked-spectrum samples, and $\alpha_{\text {thick }}$ (right panel) for the highfrequency peaked-spectrum sample that have reported spectroscopic redshifts. The color of each point corresponds to the redshift of the source. The median uncertainties for the various parameters are plotted in the corner of the diagrams.
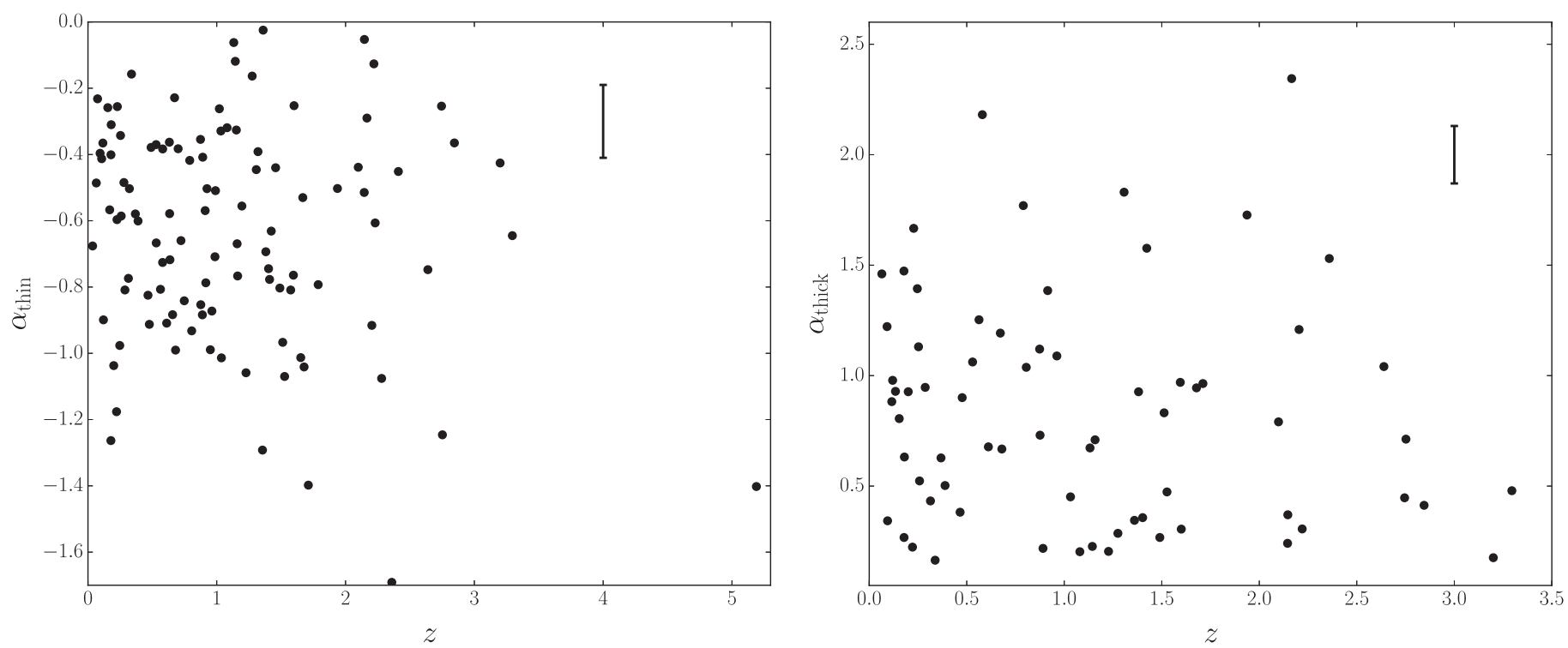

Figure 20. The variation of redshift with $\alpha_{\text {thin }}$ (left panel) for the high- and low-frequency peaked-spectrum samples, and $\alpha_{\text {thick }}$ (right panel) for the high-frequency peaked-spectrum sample. The median uncertainty for the respective spectral index is plotted in the top right corner of the diagrams.

The relationships between the observed spectral indices with redshift are provided in Figure 20. As shown by other authors (e.g., Roettgering et al. 1997), there is a slight trend of steeper optically thin slopes with higher redshift. The steepening of the optically thin spectral index has been variously explained to be due to increasing importance of inverse-Compton losses off the cosmic microwave background photons in the early universe or due to first-order Fermi accelerations in the hotspots when encountering a dense medium (Klamer et al. 2006). It is also evident in the right panel of Figure 20 that there is no dependence of $\alpha_{\text {thick }}$ on redshift, which is further indicative that the slope of the optically thick spectral index is dependent on the individual physics of a source.

\section{3. $5 \mathrm{GHz}$ Radio Power Relationships}

We used the 110 sources from the high- and low-frequency peaked-spectrum samples that have spectroscopic redshifts to investigate the distribution of the $5 \mathrm{GHz}$ luminosity and its dependence on redshift. The comparison frequency of $5 \mathrm{GHz}$ was chosen because past literature samples of GPS and CSS sources, such as those of O'Dea (1998) and Snellen et al. (1998), had their radio luminosities evaluated at $5 \mathrm{GHz}$. The $5 \mathrm{GHz}$ radio luminosities $P_{5 \mathrm{GHz}}$ was calculated as

$$
P_{5 \mathrm{GHz}}=4 \pi D_{L}^{2} S_{5 \mathrm{GHz}}(1+z)^{-\left(1+\alpha_{\text {thin }}\right)},
$$

where $D_{L}$ is the luminosity distance in the adopted cosmological model, $S_{5 \mathrm{GHz}}$ is the flux density of the source at $5 \mathrm{GHz}$, and the term $(1+z)^{-\left(1+\alpha_{\text {thin }}\right)}$ is the $k$-correction used in radio astronomy. $S_{5 \mathrm{GHz}}$ was evaluated by assuming the fit of Equation (3), in particular $\alpha_{\text {thin }}$ derived from that fit, is an accurate description of a peaked-spectrum source's spectrum to $5 \mathrm{GHz}$. We note that it is possible that the spectrum of a source could deviate from the power-law description identified between the peak frequency and $843 \mathrm{MHz} / 1.4 \mathrm{GHz}$ by 


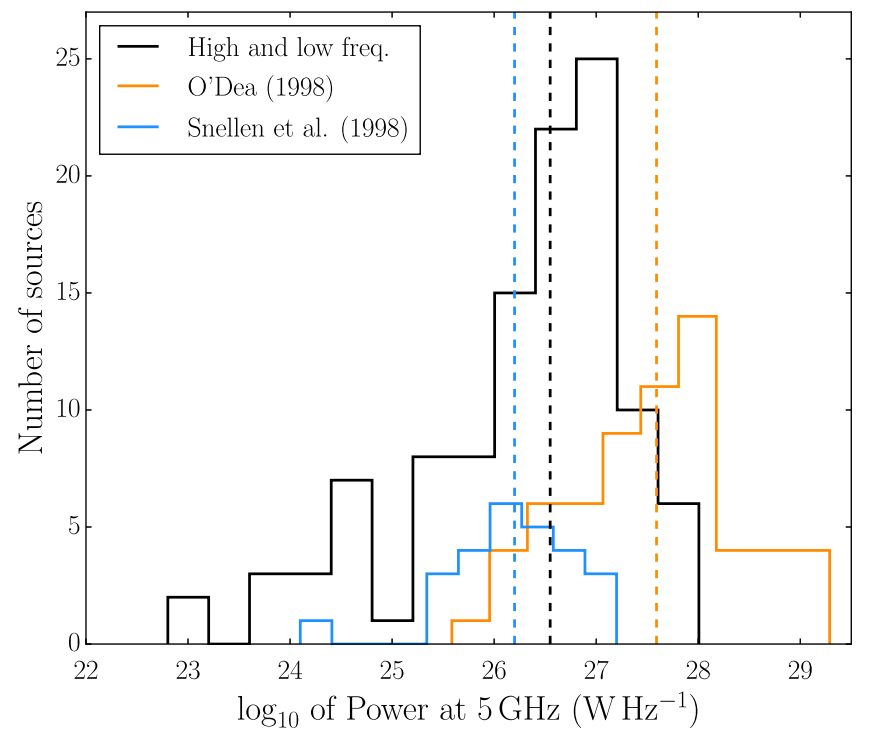

Figure 21. Distribution of the $5 \mathrm{GHz}$ radio power for the 110 sources from the low- and high-frequency peaked-spectrum samples that have reported spectroscopic redshifts is shown in black. The orange and blue histograms represent the $5 \mathrm{GHz}$ power distribution of the O'Dea (1998) sample and Snellen et al. (1998) sample, respectively. The median $P_{5 \mathrm{GHz}}$ values, and the range to the 16th and 84th percentiles of the distribution, for samples identified by O'Dea (1998), Snellen et al. (1998), and this study, are 27.6 $6_{-1.0}^{+0.7}, 26.2_{-0.4}^{+0.5}$, and $26.5_{-1.2}^{+0.7}$ $\left(\log _{10} \mathrm{~W} \mathrm{~Hz}^{-1}\right)$, respectively. The median values are plotted as dashed lines in each respective sample's color.

$5 \mathrm{GHz}$, either due to a high-frequency spectral break or because the emission from the radio core begins to exceed the radio lobe emission. However, such deviations are not expected to be significant until frequencies greater than $5 \mathrm{GHz}$ (Chhetri et al. 2012).

The distribution of the $5 \mathrm{GHz}$ radio power for the high- and low-frequency peaked-spectrum samples and the variation with redshift is provided in Figures 21 and 22, respectively. The sample presented by Snellen et al. (1998), as shown by the blue histogram and squares, has sources with similar $5 \mathrm{GHz}$ radio luminosities as the peaked-spectrum samples presented in this study. However, our low- and high-frequency peaked-spectrum sample spans a wider range in luminosity than the sample identified by Snellen et al. (1998). Additionally, our lowfrequency peaked-spectrum sample contains the weakest peaked-spectrum source ever identified with $P_{5 \mathrm{GHz}}=$ $6.3 \times 10^{22} \mathrm{~W} \mathrm{~Hz}^{-1}$.

When compared to the GPS and CSS sample presented by O'Dea (1998), as shown by the orange histogram and triangles in Figures 21 and 22, the peaked-spectrum sources identified in this study are on average an order of magnitude fainter at $5 \mathrm{GHz}$, and there are no peaked-spectrum sources in the high or low frequency samples that exceed a $5 \mathrm{GHz}$ power of $1 \times 10^{28} \mathrm{~W} \mathrm{~Hz}^{-1}$. The selection criteria employed in this study do not bias against selecting sources at $P_{5 \mathrm{GHz}}>$ $10^{28} \mathrm{~W} \mathrm{~Hz}^{-1}$. Therefore, the low and high frequency peakedspectrum samples lack the similar highly luminous sources found in the O'Dea (1998) sample because of evolutionary or redshift effects, or some combination of both.

It is expected from various evolutionary models of CSS and GPS sources (e.g., Snellen et al. 2000; Kunert-Bajraszewska et al. 2010) that the luminosity of a source declines with increasing linear size, which also corresponds to a shift of

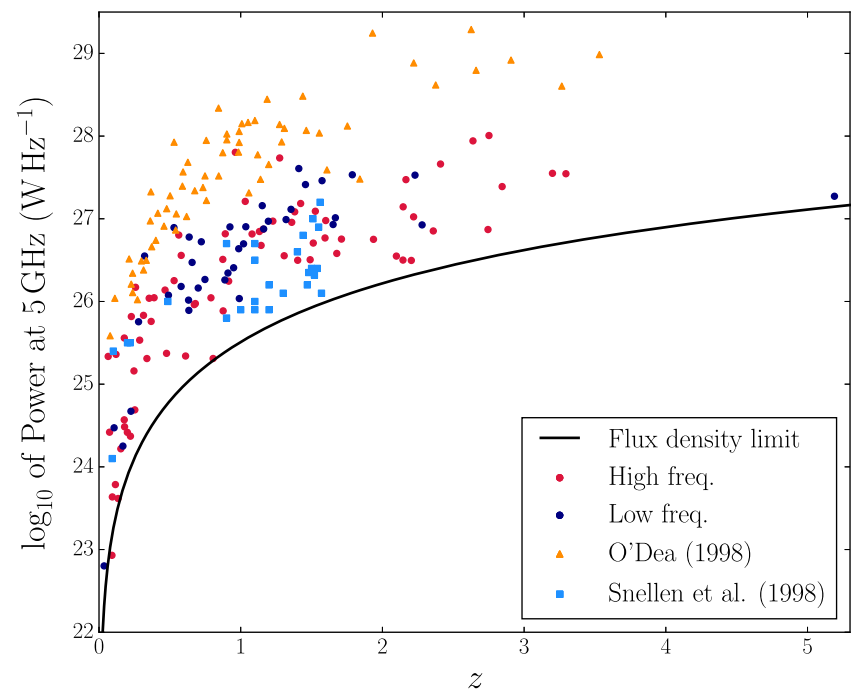

Figure 22. $5 \mathrm{GHz}$ radio power against redshift for the 110 sources from the low- and high-frequency peaked-spectrum samples that have reported spectroscopic redshifts. The sources from the high- and low-frequency samples are shown as red and navy circles, respectively. The orange triangles and light blue squares represent the GPS and CSS sources from the O'Dea (1998) and Snellen et al. (1998) samples, respectively. The black line corresponds to the $5 \mathrm{GHz}$ luminosity limit for a source that has a peak flux density of $0.16 \mathrm{Jy}$ at $230 \mathrm{MHz}$, approximately the flux density cut employed in the selection process, assuming the median optically thin spectral index of -0.77 .

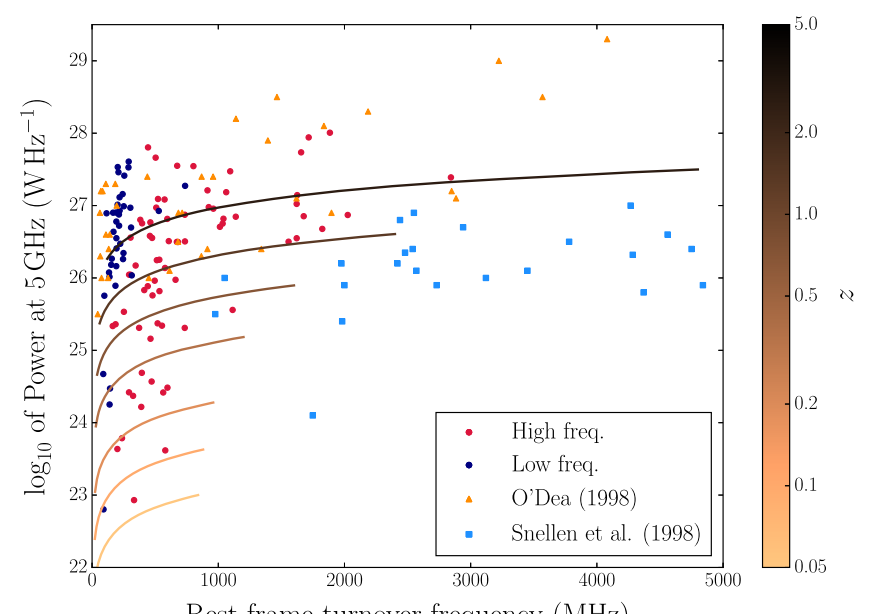

Rest frame turnover frequency $(\mathrm{MHz})$

Figure 23. The variation in $5 \mathrm{GHz}$ radio power with the rest frame turnover frequency for the high and low frequency peaked-spectrum samples, shown by red and navy circles, respectively. The orange triangles and blue squares represent the GPS and CSS sources from the O'Dea (1998) and Snellen et al. (1998) samples, respectively. The colored curves are the limit in which peakedspectrum sources at various redshifts will be identified in this study, assuming the minimum peak flux density of $0.16 \mathrm{Jy}$, since the selection process is only sensitive to turnovers between 72 and $\approx 800 \mathrm{MHz}$. The color bar corresponds to the redshift at which the limit is evaluated, with each curve from bottom to top corresponding to redshifts $0.05,0.1,0.2,0.5,1,2$, and 5 . Note that a number of sources from the O'Dea (1998) and Snellen et al. (1998) samples extend to rest frame frequencies above $5000 \mathrm{MHz}$.

the intrinsic turnover frequency to lower frequencies. The dependence of the $5 \mathrm{GHz}$ luminosity for the low and high frequency peaked-spectrum samples on rest frame turnover frequency, also including the samples presented by Snellen et al. (1998) and O'Dea (1998), is presented in Figure 23. Note that the general shape of the distribution, with no sources with low power and high rest frame turnover in the high or low 
frequency peaked-spectrum samples, is a product of redshift evolution of the flux density limit and also results from the selection process which is only sensitive to peaked-spectrum sources that display an observed spectral turnover between 72 and $\approx 800 \mathrm{MHz}$. Such redshift evolution of the flux density and turnover frequency limits is communicated by the colored curves in Figure 23.

It is promising that there are a number of sources from the low- and high-frequency peaked-spectrum samples that have $P_{5 \mathrm{GHz}}<10^{25} \mathrm{~W} \mathrm{~Hz}^{-1}$ and rest-frame turnover frequencies lower than $1 \mathrm{GHz}$, consistent with the evolutionary path of a self-similar evolving, ram-pressure-confined radio source (Kaiser \& Alexander 1997; Snellen et al. 2000). However, there is a large number of peaked-spectrum sources that have $P_{5 \mathrm{GHz}}>10^{26} \mathrm{~W} \mathrm{~Hz}^{-1}$ and rest-frame turnover frequencies lower than $1 \mathrm{GHz}$, which is inconsistent with this evolutionary model for peaked-spectrum sources. It is possible that the sources with high power and low intrinsic turnover could be confined to small spatial scales due to a dense ambient medium, or they could be jet-dominated sources, as opposed to the sources at lower power being lobe dominated. Future studies are required to test if the optical hosts of these sources with high power and low intrinsic turnover are quasars or if their linear sizes are too small for the respective turnover frequency, as expected from SSA theory.

It is also possible that the surplus of sources with high power and low intrinsic turnover frequency could be the product of the method of assembling the redshift information of the peaked-spectrum sources from inhomogeneous literature sources, each with varying degrees of completeness. To confirm the trend of lower power with lower intrinsic turnover frequencies, it is vital to repeat this analysis using a spectroscopic redshift survey with known completeness, such as 6dFGS and SDSS, and to then produce a local luminosity function.

\section{Extreme Spectra}

As is evident from the right panel of Figure 14, there are approximately 15 sources that have a reliable optically thick spectral index near or above the theoretical SSA limit of 2.5 (Pacholczyk 1970). The spectra for 6 of the brightest sources that have an optically thick spectral index near or exceeding 2.5 are shown in Figure 24, with the fit of the general curved model, SSA, and homogeneous FFA model shown in black, purple, and red, respectively.

The values of $\alpha_{\text {thick }}$ and the peak frequency $\nu_{\mathrm{p}}$ from Equation (3) for the six sources are provided in Table 3. Because the spectral index below the turnover is very steep, we also fit the homogeneous FFA model of Equation (4) since it provides exponential attenuation at low frequencies, and a SSA model by forcing $\alpha_{\text {thick }}=2.5$ in Equation (3). The reduced $\chi^{2}$ value for the model fits are also provided in Table 3. While the SSA model provides similar fitting statistics, the FFA model is statistically favored over the SSA model for all the sources, excluding GLEAM J100256-354157. None of these sources have been previously identified as peaked-spectrum sources and most lack optical or higher radio frequency counterparts.

If these peaked-spectrum sources are confirmed to have similar physical morphologies as the HFP, GPS, and CSS sources that are characterized as young, such as core-lobe structures on milliarcsecond scales, the spectra presented in
Figure 24 could be the first conclusive evidence of FFA acting in previously identified young sources. Such a detection of FFA absorption would unambiguously imply that at least a portion of the HFP, GPS, and CSS population is confined to small spatial scales by a dense ambient nuclear medium.

Alternatively, these sources could be jet dominated, with the FFA absorption occurring as the radio emission from the jet passes through a dense circumnuclear medium. Such a dense circumnuclear medium, possibly associated with the broad line region (Lobanov 1998), has been suggested to exist in Centaurus A (Jones et al. 1996), Cygnus A (Krichbaum et al. 1998; Tingay \& Murphy 2001), and 3C 84 (Walker et al. 1994) because the counter-jets in these sources are significantly fainter than the forward jet or are not observed at all. The only source that has a AT20G and optical counterpart, GLEAM J144815-162024, is associated with a quasar (Mahony et al. 2011) and has a linear size smaller than $0.5 \mathrm{kpc}$ based on its $6 \mathrm{~km} 20 \mathrm{GHz}$ visibilities (Chhetri et al. 2013). Jetdominated sources have been shown to display low-frequency variability on timescales of several months (Bell et al. 2014), so it is vital to assess if these sources are variable before fitting multiepoch data since the trends induced by variability may dominate physical relationships.

Finally, it is possible that such spectra are the product of an extreme scattering event (ESE), since ESEs haven been shown to produce irregular spectra and steep spectral slopes (e.g., Bannister et al. 2016). An ESE is likely due to the propagation of a compact source's radio emission through dense plasma lenses in the Galaxy (Fiedler et al. 1994). The densities required to produce such extreme lensing are inconsistent with our current understanding of gas conditions in the Milky Way. In particular, GLEAM J100256-354157 is more likely a potential ESE than the other sources presented in Figure 24, as the spectrum is even inconsistent with FFA absorption. However, the spectral properties of ESEs at low frequencies are unknown, with limited variability seen below $1 \mathrm{GHz}$ (Bannister et al. 2016). The low frequencies provided by the GLEAM survey could place the most stringent constraints on the density of the interstellar medium if a source is shown to be experiencing an ESE.

During the first year of the GLEAM survey, many sources were observed on multiple occasions due to the wide field of view of the MWA and the highly degenerate observing strategy. This allows us to place constraints on the variability of some of these sources below $231 \mathrm{MHz}$. GLEAM J001513472706, GLEAM J074211-673309, and GLEAM J144815162024 were observed by the MWA twice with a gap of three months, and GLEAM J211949-343936 and GLEAM J213024434819 were observed twice with a gap of nine months. There is no evidence of variability in the low-frequency spectra for any of the sources. Unfortunately, the best ESE candidate, GLEAM J100256-354157, was only observed once during the GLEAM survey.

Independent of the nature of these sources, they represent excellent candidates for targeted follow -up observations to observe intrinsic $\mathrm{H} \mathrm{I}$ absorption due to the inference of a dense ambient medium from the spectrum. To assess the nature of these sources, we require low-frequency monitoring to constrain any variability and high-resolution observations with LOFAR or the GMRT. We note that the optically thick slope is completely determined from the contemporaneous GLEAM 

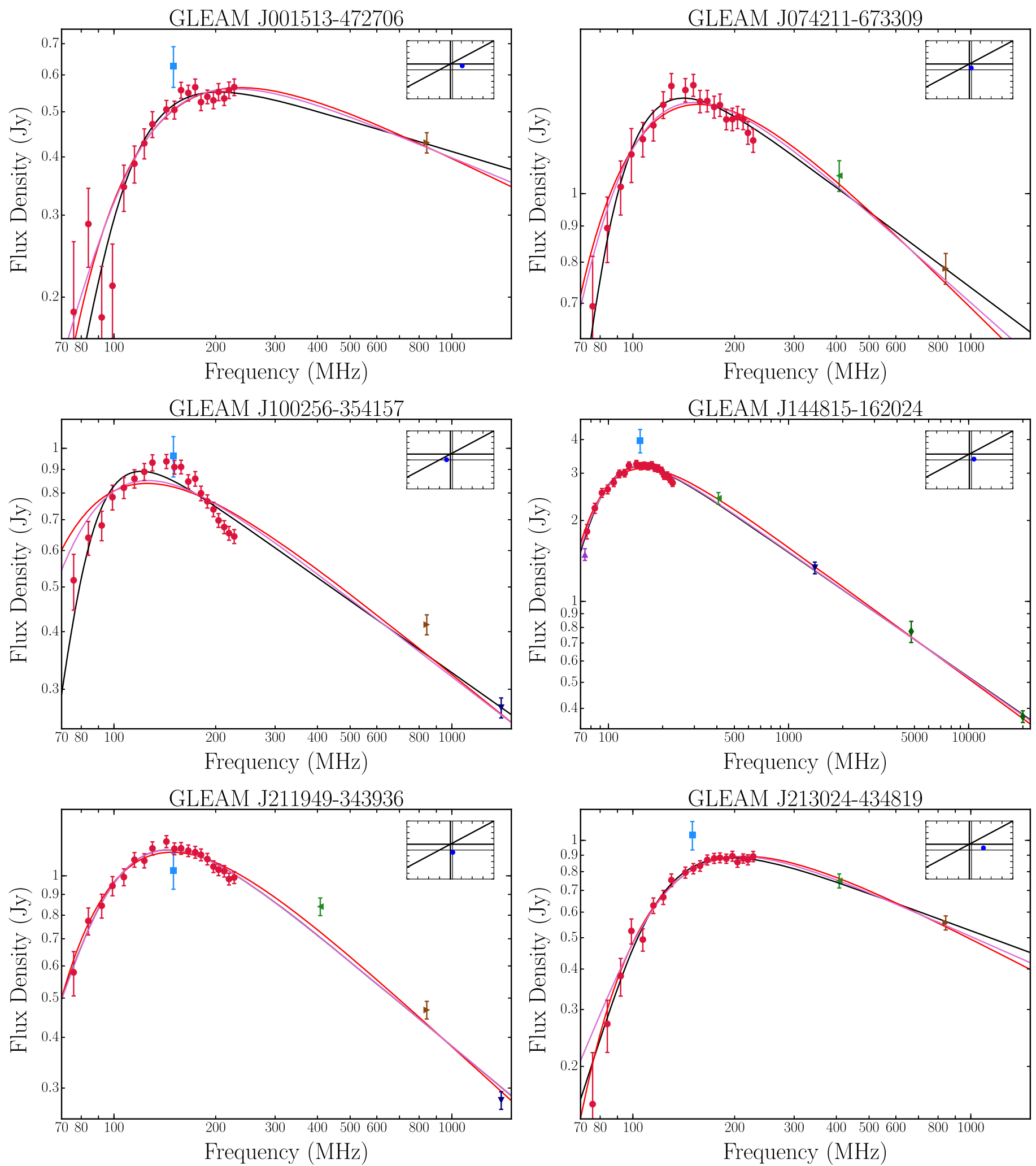

Figure 24. The spectra of six extreme peaked-spectrum sources that have $\alpha_{\text {thick }} \gtrsim 2.5$. The symbols represent the same survey data as in Figure 7. The black, purple, and red curves represent the fit of the general curved model described by Equation (3), homogeneous SSA, and the single homogeneous FFA model of Equation (4), respectively. In all cases, the FFA model is the statistically favored model. Note that the plot for GLEAM J144815-162024 extends to $20 \mathrm{GHz}$ since it has AT20G measurements represented by the green diamonds. The inset plot located in the top right corner of each plot displays the position of the source in the color-color diagram of Figure 2 by a blue circle.

data, therefore the extreme spectral slope cannot be due to variability. In particular, observations with the ATCA or VLA above the turnover will be important to ensure that the spectral width is properly sampled, providing an additional parameter for model differentiation, as shown by Callingham et al. (2015), and confirmation of the extreme spectral slope. 
Table 3

A List of the Parameters Derived from the Spectral Fits of the General Curved Model, SSA, and FFA Models to the Specra Presented in Figure 24

\begin{tabular}{lcccc}
\hline \hline GLEAM Name & $\alpha_{\text {thick }}$ & $\nu_{\mathrm{p}}(\mathrm{MHz})$ & $\chi_{\text {red,SSA }}^{2}$ & $\chi_{\text {red,FFA }}^{2}$ \\
\hline J001513-472706 & $3.7 \pm 1.1$ & $240 \pm 20$ & 1.31 & 1.24 \\
J074211-673309 & $4.1 \pm 0.9$ & $160 \pm 30$ & 1.06 & 0.81 \\
J100256-354157 & $5.0 \pm 1.7$ & $110 \pm 40$ & 5.40 & 4.52 \\
J144815-162024 & $2.6 \pm 0.2$ & $160 \pm 10$ & 1.10 & 0.80 \\
J211949-343936 & $2.5 \pm 0.3$ & $150 \pm 20$ & 1.29 & 1.25 \\
J213024-434819 & $3.2 \pm 0.6$ & $220 \pm 30$ & 0.80 & 0.70 \\
\hline
\end{tabular}

Note. The parameters listed in the table are the optically thick spectral index $\alpha_{\text {thick }}$ from the fit of Equation (3), spectral turnover frequency $\nu_{\mathrm{p}}$ from the fit of Equation (3), and the reduced $\chi^{2}$-value of the model fit for FFA and SSA $\chi_{\text {red,FFA }}^{2}$ and $\chi_{\text {red,SSA }}^{2}$, respectively.

\section{Conclusions}

In this study, we identify 1483 peaked-spectrum sources that display a spectral turnover between $72 \mathrm{MHz}$ and $1.4 \mathrm{GHz}$, representing $\approx 4.5 \%$ of the GLEAM radio source population. A source was selected as peaked-spectrum source when it either displayed a spectral peak within the GLEAM band, a concave spectrum in radio color-color phase space when a SUMSS or NVSS flux density measurement was added to the spectrum, or a positive power-law that extended from the top of the GLEAM band to a SUMSS or NVSS counterpart. The peaked-spectrum candidates were also required to be unresolved in the GLEAM extragalactic catalog, located between the declinations of $-80^{\circ}$ and $+30^{\circ}$, and brighter than $0.16 \mathrm{Jy}$ in the GLEAM wideband image, which is centered at $200 \mathrm{MHz}$. Using the wide fractional bandwidth of the GLEAM survey as a basis of identifying peaked-spectrum sources implies that the observations at and below the spectral peak are minimally affected by variability.

All of the 73 known GPS, CSS, and HFP sources that have a spectral turnover between $72 \mathrm{MHz}$ and $1.4 \mathrm{GHz}$ and are bright enough to be detected in the GLEAM survey are contained within our peaked-spectrum sample, demonstrating the high reliability of the selection criteria. This study provides 1410 new peaked-spectrum sources, representing an increase of over a factor of six in the number of peaked-spectrum sources known below a declination of $+30^{\circ}$. We also highlight that six of the GPS sources that peak at $5 \mathrm{GHz}$ or above display a convex spectrum between the GLEAM survey and NVSS/ SUMSS, implying that at least a portion of the GPS and HFP population is likely to go through multiple epochs of activity.

The spectra of a number of USS sources are found to be turning over at GLEAM frequencies. We suggest that a steep optically thin spectral index and a spectral peak below $230 \mathrm{MHz}$ are potential indicators of high-redshift candidates since both spectral properties could be produced by radio lobes and hot spots being confined by dense ambient nuclear region. All of the sources with a reported spectroscopic redshift that have an optically thin spectral index steeper than -1.2 and a turnover frequency in the GLEAM band is located at redshifts greater than two. However, this trend needs to be confirmed with optical or H I absorption follow-up campaigns.

In terms of the spectral properties of the peaked-spectrum sample, the distributions of the optically thin and thick spectral indices are similar to previous studies, but with a much wider spread, which is mostly due to the high sensitivity of SUMSS/ NVSS and the wide fractional bandwidth of the GLEAM survey. In particular, the large spread in the optically thick spectral indices indicates that the turnover in the spectrum is caused by an inhomogeneous environment that differs from source to source. There is also no dependence of the observed turnover frequency on redshift, as expected if the sources selected with observed low-frequency spectral turnovers were composed of both local CSS sources and high-redshift GPS sources. To measure linear sizes and decouple these two source populations, high-resolution follow-up measurements at low frequencies, for example with the GMRT or LOFAR, are required.

The $5 \mathrm{GHz}$ luminosity distribution of the peaked-spectrum sample lacks the brightest GPS and CSS sources of previous samples, suggesting a convolution of evolution and redshift effects for sources identified at low frequencies relative to peaked-spectrum sources selected above $1 \mathrm{GHz}$. While the trends evident in intrinsic turnover frequency and radio luminosity are consistent with redshift evolution, there are a number of sources at high power and low observed turnover frequencies that are inconsistent with the stereotypical evolutionary model of a peaked-spectrum source. The small-scale structure and optical properties of these sources need to be investigated to confirm whether these sources have the same properties as young precursors to large radio galaxies.

Finally, we highlight six sources that have optically thick spectral indices near or above the SSA limit. If the sources are shown to be minimally variable and have milliarcsecond scale double morphologies, then this detection is the first clear violation of SSA theory in GPS, CSS, and HFP sources. Independently, these sources represent excellent candidates for targeted follow-up surveys to observe intrinsic H I absorption that is due to the inference of a dense ambient medium.

This scientific work makes use of the Murchison Radioastronomy Observatory, operated by CSIRO. We acknowledge the Wajarri Yamatji people as the traditional owners of the Observatory site. Support for the operation of the MWA is provided by the Australian Government (NCRIS), under a contract to Curtin University administered by Astronomy Australia Limited. We acknowledge the Pawsey Supercomputing Centre, which is supported by the Western Australian and Australian Governments. This research was conducted by the Australian Research Council Centre of Excellence for Allsky Astrophysics (CAASTRO), through project number CE110001020. J.R.C. acknowledges the support of the Australian Postgraduate Award. The Dunlap Institute is funded through an endowment established by the David Dunlap family and the University of Toronto. B.M.G. acknowledges the support of the Natural Sciences and Engineering Research Council of Canada (NSERC) through grant RGPIN-201505948, and of the Canada Research Chairs program. This research has made use of the NASA/IPAC Extragalactic Database (NED), which is operated by the Jet Propulsion Laboratory, California Institute of Technology, under contract with the National Aeronautics and Space Administration, and the VizieR catalog access tool, CDS, Strasbourg, France. Topcat (Taylor 2005), SAOImage DS9, NASA's Astrophysics Data System bibliographic services, and Astropy, a community-developed core Python package for Astronomy (Astropy Collaboration et al. 2013), were also used in this study. 


\section{Appendix A \\ List of Column Headings for Tables of the Peaked- spectrum and Convex Source Samples}

The column numbers, names, and units for the tables presenting the different peaked-spectrum source samples are outlined below. The tables are available online. The optically thick spectral index is not presented for sources that have a spectral peak below $100 \mathrm{MHz}$ because the values have large uncertainties. The parameters derived from the generic curvature model of Equation (3), such as $S_{\mathrm{p}}, \nu_{\mathrm{p}}, \alpha_{\text {thin }}$, and $\alpha_{\text {thick}}$, are not reported for the GPS sample, for the convex source sample, and for the sources that peak below $72 \mathrm{MHz}$. Redshift values are not listed for sources that peak below $72 \mathrm{MHz}$ or the convex source sample. We note that all of the sub-band GLEAM flux densities are presented, but only flux densities that had a $\mathrm{S} / \mathrm{N}>3$ are used in the spectral fitting and plotted in the corresponding source spectra.

\begin{tabular}{|c|c|c|c|}
\hline Number & Name & Unit & Description \\
\hline 1 & GLEAM name & $\cdots$ & $\begin{array}{l}\text { Name of the source in the GLEAM } \\
\text { extragalactic catalog }\end{array}$ \\
\hline 2 & GLEAM R.A. & degrees & $\begin{array}{l}\text { R.A. of the source in the the GLEAM } \\
\text { extragalactic catalog }\end{array}$ \\
\hline 3 & GLEAM Decl. & degrees & $\begin{array}{l}\text { Decl. of the source in the GLEAM extra- } \\
\text { galactic catalog }\end{array}$ \\
\hline 4 & $S_{\mathrm{p}}$ & Jy & Flux density at the spectral peak \\
\hline 5 & $\Delta S_{\mathrm{p}}$ & Jy & $\begin{array}{l}\text { Uncertainty in the spectral peak flux } \\
\text { density }\end{array}$ \\
\hline 6 & $\nu_{\mathrm{p}}$ & $\mathrm{MHz}$ & Frequency of the spectral peak \\
\hline 7 & $\Delta \nu_{\mathrm{p}}$ & $\mathrm{MHz}$ & Uncertainty in the spectral peak frequency \\
\hline 8 & $\alpha_{\text {thin }}$ & $\cdots$ & $\begin{array}{l}\text { Optically thin spectral index from fitting } \\
\text { Equation (3) to the entire spectrum }\end{array}$ \\
\hline 9 & $\Delta \alpha_{\text {thin }}$ & $\cdots$ & $\begin{array}{l}\text { Uncertainty in the optically thin spectral } \\
\text { index }\end{array}$ \\
\hline 10 & $\alpha_{\text {thick }}$ & $\cdots$ & $\begin{array}{l}\text { Optically thick spectral index from fitting } \\
\text { Equation (3) to the entire spectrum }\end{array}$ \\
\hline 11 & $\Delta \alpha_{\text {thick }}$ & $\cdots$ & $\begin{array}{l}\text { Uncertainty in the optically thick spectral } \\
\text { index }\end{array}$ \\
\hline 12 & $\alpha_{\text {low }}$ & $\cdots$ & $\begin{array}{l}\text { Low-frequency spectral index derived } \\
\text { from fitting a power law to data } \\
\text { between } 72 \text { and } 231 \mathrm{MHz}\end{array}$ \\
\hline 13 & $\Delta \alpha_{\text {low }}$ & $\cdots$ & $\begin{array}{l}\text { Uncertainty in the low-frequency spectral } \\
\text { index }\end{array}$ \\
\hline 14 & $\alpha_{\text {high }}$ & $\cdots$ & $\begin{array}{l}\text { High frequency spectral index derived } \\
\text { from fitting a power law } \\
\text { to SUMSS and/or NVSS flux density } \\
\text { point(s) and to GLEAM data at } 189 \text { and } \\
212 \mathrm{MHz}\end{array}$ \\
\hline 15 & $\Delta \alpha_{\text {high }}$ & $\cdots$ & $\begin{array}{l}\text { Uncertainty in the high-frequency spectral } \\
\text { index }\end{array}$ \\
\hline 16 & $q$ & $\ldots$ & $\begin{array}{l}\text { Curvature parameter derived from fitting } \\
\text { Equation (2) to only the GLEAM data }\end{array}$ \\
\hline 17 & $\Delta q$ & $\ldots$ & Uncertainty in the curvature parameter \\
\hline 18 & $z$ & $\ldots$ & Spectroscopic redshift \\
\hline 19 & Ref. $z$ & $\ldots$ & $\begin{array}{l}\text { Reference for the reported spectroscopic } \\
\text { redshift }\end{array}$ \\
\hline 20 & $S_{200 \mathrm{MHz}, \text { wide }}$ & Jy & $\begin{array}{l}\text { Integrated flux density measurement of the } \\
\text { source in the GLEAM wideband image }\end{array}$ \\
\hline 21 & $\Delta S_{200 \mathrm{MHz}, \text { wide }}$ & Jy & $\begin{array}{l}\text { Uncertainty in the GLEAM wideband flux } \\
\text { density measurement }\end{array}$ \\
\hline 22 & VLSSr R.A. & degrees & R.A. of the source in VLSSr \\
\hline 23 & VLSSr Decl. & degrees & Decl. of the source in VLSSr \\
\hline 24 & $S_{\mathrm{VLSSr}}$ & Jy & VLSSr flux density at $74 \mathrm{MHz}$ \\
\hline 25 & $\Delta S_{\mathrm{VLSS} r}$ & Jy & $\begin{array}{l}\text { Uncertainty in the VLSSr flux density } \\
\text { measurement, convolved with calibra- } \\
\text { tion uncertainty }\end{array}$ \\
\hline
\end{tabular}

\begin{tabular}{|c|c|c|c|}
\hline \multicolumn{4}{|c|}{ (Continued) } \\
\hline Number & Name & Unit & Description \\
\hline 26 & $\begin{array}{l}\text { TGSS- } \\
\text { ADR1 name }\end{array}$ & $\ldots$ & Name of the source in TGSS-ADR1 \\
\hline 27 & $\begin{array}{l}\text { TGSS-ADR1 } \\
\text { R.A. }\end{array}$ & degrees & R.A. of the source in TGSS-ADR1 \\
\hline 28 & $\begin{array}{l}\text { TGSS- } \\
\text { ADR1 Decl. }\end{array}$ & degrees & Decl. of the source in TGSS-ADR1 \\
\hline 29 & $S_{\mathrm{TGSS}}$ & Jy & TGSS-ADR1 flux density at $150 \mathrm{MHz}$ \\
\hline 30 & $\Delta S_{\mathrm{TGSS}}$ & Jy & $\begin{array}{l}\text { Uncertainty in the TGSS-ADR1 flux } \\
\text { density measurement }\end{array}$ \\
\hline 31 & MRC name & $\ldots$ & Name of the source in MRC \\
\hline 32 & MRC R.A. & degrees & R.A. of the source in MRC \\
\hline 33 & MRC Decl. & degrees & Decl. of the source in MRC \\
\hline 34 & $S_{\mathrm{MRC}}$ & Jy & MRC flux density at $408 \mathrm{MHz}$ \\
\hline 35 & $\Delta S_{\mathrm{MRC}}$ & Jy & $\begin{array}{l}\text { Uncertainty in the MRC flux density } \\
\text { measurement, convolved with calibra- } \\
\text { tion uncertainty }\end{array}$ \\
\hline 36 & SUMSS R.A. & degrees & R.A. of the source in SUMSS \\
\hline 37 & SUMSS Decl. & degrees & Decl. of the source in SUMSS \\
\hline 38 & $S_{\text {SUMSS }}$ & Jy & SUMSS flux density at $843 \mathrm{MHz}$ \\
\hline 39 & $\Delta S_{\text {SUMSS }}$ & Jy & $\begin{array}{l}\text { Uncertainty in the SUMSS flux density } \\
\text { measurement, convolved with calibra- } \\
\text { tion uncertainty }\end{array}$ \\
\hline 40 & NVSS R.A. & degrees & R.A. of the source in NVSS \\
\hline 41 & NVSS Decl. & degrees & Decl. of the source in NVSS \\
\hline 42 & $S_{\mathrm{NVSS}}$ & Jy & NVSS flux density at $1.4 \mathrm{GHz}$ \\
\hline 43 & $\Delta S_{\mathrm{NVSS}}$ & Jy & $\begin{array}{l}\text { Uncertainty in the NVSS flux density } \\
\text { measurement, convolved with calibra- } \\
\text { tion uncertainty }\end{array}$ \\
\hline 44 & $S_{76}$ & Jy & $\begin{array}{l}\text { Integrated flux density at } 76 \mathrm{MHz} \\
\text { from the GLEAM extragalactic } \\
\text { catalog }\end{array}$ \\
\hline 45 & $\Delta S_{76}$ & Jy & $\begin{array}{l}\text { Uncertainty in the integrated flux density } \\
\text { at } 76 \mathrm{MHz} \text {, which is the convolution of } \\
\text { fitting and systematic errors }\end{array}$ \\
\hline 46 & $S_{84}$ & Jy & $\begin{array}{l}\text { Integrated flux density at } 84 \mathrm{MHz} \text { from } \\
\text { the GLEAM extragalactic catalog }\end{array}$ \\
\hline 47 & $\Delta S_{84}$ & Jy & $\begin{array}{l}\text { Uncertainty in the integrated flux density } \\
\text { at } 84 \mathrm{MHz} \text {, which is the convolution of } \\
\text { fitting and systematic errors }\end{array}$ \\
\hline 48 & $S_{92}$ & Jy & $\begin{array}{l}\text { Integrated flux density at } 92 \mathrm{MHz} \text { from } \\
\text { the GLEAM extragalactic catalog }\end{array}$ \\
\hline 49 & $\Delta S_{92}$ & Jy & $\begin{array}{l}\text { Uncertainty in the integrated flux } \\
\text { density at } 92 \mathrm{MHz} \text {, which is the } \\
\text { convolution of fitting and systematic } \\
\text { errors }\end{array}$ \\
\hline 50 & $S_{99}$ & Jy & $\begin{array}{l}\text { Integrated flux density at } 99 \mathrm{MHz} \text { from } \\
\text { the GLEAM extragalactic catalog }\end{array}$ \\
\hline 51 & $\Delta S_{99}$ & Jy & $\begin{array}{l}\text { Uncertainty in the integrated flux density } \\
\text { at } 99 \mathrm{MHz} \text {, which is the convolution of } \\
\text { fitting and systematic errors }\end{array}$ \\
\hline 52 & $S_{107}$ & Jy & $\begin{array}{l}\text { Integrated flux density at } 107 \mathrm{MHz} \text { from } \\
\text { the GLEAM extragalactic catalog }\end{array}$ \\
\hline 53 & $\Delta S_{107}$ & Jy & $\begin{array}{l}\text { Uncertainty in the integrated flux density } \\
\text { at } 107 \mathrm{MHz} \text {, which is the convolution } \\
\text { of fitting and systematic errors }\end{array}$ \\
\hline 54 & $S_{115}$ & Jy & $\begin{array}{l}\text { Integrated flux density at } 115 \mathrm{MHz} \text { from } \\
\text { the GLEAM extragalactic catalog }\end{array}$ \\
\hline 55 & $\Delta S_{115}$ & Jy & $\begin{array}{l}\text { Uncertainty in the integrated flux density } \\
\text { at } 115 \mathrm{MHz} \text {, which is the convolution } \\
\text { of fitting and systematic errors }\end{array}$ \\
\hline 56 & $S_{122}$ & Jy & $\begin{array}{l}\text { Integrated flux density at } 122 \mathrm{MHz} \text { from } \\
\text { the GLEAM extragalactic catalog }\end{array}$ \\
\hline 57 & $\Delta S_{122}$ & Jy & $\begin{array}{l}\text { Uncertainty in the integrated flux density } \\
\text { at } 122 \mathrm{MHz} \text {, which is the convolution } \\
\text { of fitting and systematic errors }\end{array}$ \\
\hline
\end{tabular}


(Continued)

\begin{tabular}{|c|c|c|c|}
\hline Number & Name & Unit & Description \\
\hline 58 & $S_{130}$ & Jy & $\begin{array}{l}\text { Integrated flux density at } 130 \mathrm{MHz} \text { from } \\
\text { the GLEAM extragalactic catalog }\end{array}$ \\
\hline 59 & $\Delta S_{130}$ & Jy & $\begin{array}{l}\text { Uncertainty in the integrated flux density } \\
\text { at } 130 \mathrm{MHz} \text {, which is the convolution } \\
\text { of fitting and systematic errors }\end{array}$ \\
\hline 60 & $S_{143}$ & Jy & $\begin{array}{l}\text { Integrated flux density at } 143 \mathrm{MHz} \text { from } \\
\text { the GLEAM extragalactic catalog }\end{array}$ \\
\hline 61 & $\Delta S_{143}$ & Jy & $\begin{array}{l}\text { Uncertainty in the integrated flux density } \\
\text { at } 143 \mathrm{MHz} \text {, which is the convolution } \\
\text { of fitting and systematic errors }\end{array}$ \\
\hline 62 & $S_{151}$ & Jy & $\begin{array}{l}\text { Integrated flux density at } 151 \mathrm{MHz} \text { from } \\
\text { the GLEAM extragalactic catalog }\end{array}$ \\
\hline 63 & $\Delta S_{151}$ & Jy & $\begin{array}{l}\text { Uncertainty in the integrated flux density } \\
\text { at } 151 \mathrm{MHz} \text {, which is the convolution } \\
\text { of fitting and systematic errors }\end{array}$ \\
\hline 64 & $S_{158}$ & Jy & $\begin{array}{l}\text { Integrated flux density at } 158 \mathrm{MHz} \text { from } \\
\text { the GLEAM extragalactic catalog }\end{array}$ \\
\hline 65 & $\Delta S_{158}$ & $\mathrm{Jy}$ & $\begin{array}{l}\text { Uncertainty in the integrated flux density } \\
\text { at } 158 \mathrm{MHz} \text {, which is the convolution } \\
\text { of fitting and systematic errors }\end{array}$ \\
\hline 66 & $S_{166}$ & Jy & $\begin{array}{l}\text { Integrated flux density at } 166 \mathrm{MHz} \text { from } \\
\text { the GLEAM extragalactic catalog }\end{array}$ \\
\hline 67 & $\Delta S_{166}$ & Jy & $\begin{array}{l}\text { Uncertainty in the integrated flux density } \\
\text { at } 166 \mathrm{MHz} \text {, which is the convolution } \\
\text { of fitting and systematic errors }\end{array}$ \\
\hline 68 & $S_{174}$ & Jy & $\begin{array}{l}\text { Integrated flux density at } 174 \mathrm{MHz} \text { from } \\
\text { the GLEAM extragalactic catalog }\end{array}$ \\
\hline 69 & $\Delta S_{174}$ & Jy & $\begin{array}{l}\text { Uncertainty in the integrated flux density } \\
\text { at } 174 \mathrm{MHz} \text {, which is the convolution } \\
\text { of fitting and systematic errors }\end{array}$ \\
\hline 70 & $S_{181}$ & Jy & $\begin{array}{l}\text { Integrated flux density at } 181 \mathrm{MHz} \text { from } \\
\text { the GLEAM extragalactic catalog }\end{array}$ \\
\hline 71 & $\Delta S_{181}$ & Jy & $\begin{array}{l}\text { Uncertainty in the integrated flux density } \\
\text { at } 181 \mathrm{MHz} \text {, which is the convolution } \\
\text { of fitting and systematic errors }\end{array}$ \\
\hline 72 & $S_{189}$ & Jy & $\begin{array}{l}\text { Integrated flux density at } 189 \mathrm{MHz} \text { from } \\
\text { the GLEAM extragalactic catalog }\end{array}$ \\
\hline 73 & $\Delta S_{189}$ & Jy & $\begin{array}{l}\text { Uncertainty in the integrated flux density } \\
\text { at } 189 \mathrm{MHz} \text {, which is the convolution } \\
\text { of fitting and systematic errors }\end{array}$ \\
\hline 74 & $S_{197}$ & Jy & $\begin{array}{l}\text { Integrated flux density at } 197 \mathrm{MHz} \text { from } \\
\text { the GLEAM extragalactic catalog }\end{array}$ \\
\hline 75 & $\Delta S_{197}$ & Jy & $\begin{array}{l}\text { Uncertainty in the integrated flux density } \\
\text { at } 197 \mathrm{MHz} \text {, which is the convolution } \\
\text { of fitting and systematic errors }\end{array}$ \\
\hline 76 & $S_{204}$ & Jy & $\begin{array}{l}\text { Integrated flux density at } 204 \mathrm{MHz} \text { from } \\
\text { the GLEAM extragalactic catalog }\end{array}$ \\
\hline 77 & $\Delta S_{204}$ & Jy & $\begin{array}{l}\text { Uncertainty in the integrated flux density } \\
\text { at } 204 \mathrm{MHz} \text {, which is the convolution } \\
\text { of fitting and systematic errors }\end{array}$ \\
\hline 78 & $S_{212}$ & Jy & $\begin{array}{l}\text { Integrated flux density at } 212 \mathrm{MHz} \text { from } \\
\text { the GLEAM extragalactic catalog }\end{array}$ \\
\hline 79 & $\Delta S_{212}$ & $\mathrm{Jy}$ & $\begin{array}{l}\text { Uncertainty in the integrated flux density } \\
\text { at } 212 \mathrm{MHz} \text {, which is the convolution } \\
\text { of fitting and systematic errors }\end{array}$ \\
\hline 80 & $S_{220}$ & Jy & $\begin{array}{l}\text { Integrated flux density at } 220 \mathrm{MHz} \text { from } \\
\text { the GLEAM extragalactic catalog }\end{array}$ \\
\hline 81 & $\Delta S_{220}$ & Jy & $\begin{array}{l}\text { Uncertainty in the integrated flux density } \\
\text { at } 220 \mathrm{MHz} \text {, which is the convolution } \\
\text { of fitting and systematic errors }\end{array}$ \\
\hline 82 & $S_{227}$ & Jy & $\begin{array}{l}\text { Integrated flux density at } 227 \mathrm{MHz} \text { from } \\
\text { the GLEAM extragalactic catalog }\end{array}$ \\
\hline 83 & $\Delta S_{277}$ & Jy & $\begin{array}{l}\text { Uncertainty in the integrated flux density } \\
\text { at } 227 \mathrm{MHz} \text {, which is the convolution } \\
\text { of fitting and systematic errors }\end{array}$ \\
\hline
\end{tabular}

Note. The number of the references in the table corresponds to the following studies: (0) Browne et al. (1975), (1) Jauncey et al. (1978), (2) Hunstead et al. (1978), (3) Wright et al. (1979), (4) Fricke et al. (1983), (5) Wright et al. (1983), (6) Jauncey et al. (1984), (7) Chu et al. (1986), (8) Stickel et al. (1989), (9) Hewitt \& Burbidge (1989), (10) Thompson et al. (1990), (11) Wright \& Otrupcek (1990), (12) Hewitt \& Burbidge (1991), (13) NED; (14) Singal (1993), (15) Tadhunter et al. (1993), (16) Stickel et al. (1994), (17) Heckman et al. (1994), (18) Osmer et al. (1994), (19) Cersosimo et al. (1994), (20) di Serego-Alighieri et al. (1994), (21) de Vries et al. (1995), (22) Lawrence et al. (1995), (23) Fisher et al. (1995), (24) Maza et al. (1995b), (25) Maza et al. (1995a), (26) Stickel et al. (1996), (27) McCarthy et al. (1996), (28) Snellen et al. (1996), (29) Marcha et al. (1996), (30) Zickgraf et al. (1997), (31) Roettgering et al. (1997), (32) Wiklind \& Combes (1997), (33) O’Dea \& Baum (1997), (34) Halpern \& Eracleous (1997), (35) Drinkwater et al. (1997), (36) Carilli et al. (1998), (37) de Vries et al. (1998), (38) Kapahi et al. (1998), (39) Stern et al. (1999), (40) Tonry \& Kochanek (1999), (41) van Breugel et al. (1999), (42) Best et al. (1999), (43) de Vries et al. (2000), (44) Willott et al. (2000), (45) Ellison et al. (2001), (46) De Breuck et al. (2001), (47) Jackson et al. (2002), (48) Snellen et al. (2002), (49) Colless et al. (2001), (50) Halpern et al. (2003), (51) Drake et al. (2003), (52) Liang \& Liu (2003), (53) Best et al. (2003), (54) Heidt et al. (2004), (55) Sowards-Emmerd et al. (2004), (56) Croom et al. (2004), (57) Abazajian et al. (2004), (58) Sowards-Emmerd et al. (2005), (59) Rupke et al. (2005), (60) AdelmanMcCarthy et al. (2006), (61) Tinti \& de Zotti (2006), (62) Burgess \& Hunstead (2006), (63) Barnes et al. (2001), (64) Cannon et al. (2006), (65) Labiano et al. (2007), (66) de Vries et al. (2007), (67) Adelman-McCarthy et al. (2008), (68) Healey et al. (2008), (69) Brookes et al. (2008), (70) Holt et al. (2008), (71) Gendre \& Wall (2008), (72) Mauch \& Sadler (2007), (73) Richards et al. (2009), (74) Bryant et al. (2009), (75) Schneider et al. (2010), (76) Meisner \& Romani (2010), (77) Wen et al. (2010), (78) Down et al. (2010), (79) Drinkwater et al. (2010), (80) Noterdaeme et al. (2010), (81) Hewett \& Wild (2010), (82) Ellison et al. (2010), (83) Bergeron et al. (2011), (84) Titov et al. (2011), (85) Mahony et al. (2011), (86) Chand \& Gopal-Krishna (2012), (87) Zafar et al. (2013), (88) Farnes et al. (2014), (89) Best \& Heckman (2012).

\section{Appendix B \\ Spectra of the Peaked-spectrum Sources in the High- and Low-frequency Sample, the GPS Sample, and the Sources that Have a Spectral Peak below $72 \mathrm{MHz}$}

The first 1222 components of the figure set are the spectra for all the sources that are in the high- and low-frequency peaked-spectrum samples identified in the main body of this paper. The first of these spectra is shown in Figure 25. In all cases, the GLEAM survey and NVSS/SUMSS data are presented. If the source also has a MRC, TGSS-ARD1, or VLSSr counterpart, as assessed using the cross-matching routine detailed in Section 3, the flux density from the respective survey is also presented. Overplotted on each spectrum is the best fit of the general curved model of Equation (3) to only the GLEAM, MRC, NVSS, and/or SUMSS data. For most sources, this model is an accurate description of the spectrum, but for $\approx 10 \%$ of sources it provides unphysical fits (e.g., GLEAM J032320+053413).

The next 261 components of the figure set are the spectra for all the sources that are in the GPS sample identified in the main body of this paper. In all cases, the GLEAM survey and NVSS/SUMSS data are presented. If the source also has a MRC, TGSS-ARD1, or VLSSr counterpart, as assessed using the cross-matching routine detailed in Section 3, the flux density from the respective survey is also presented. Overplotted on each spectrum is the best fit of the power-law model of Equation (1) to only the GLEAM, MRC, NVSS, and/ or SUMSS data.

The last 36 components of the figure set are the spectra for the 36 sources that have a spectral peak below $72 \mathrm{MHz}$, $q<-0.2$ and a $\mathrm{S} / \mathrm{N}$ greater than 100 in the GLEAM 


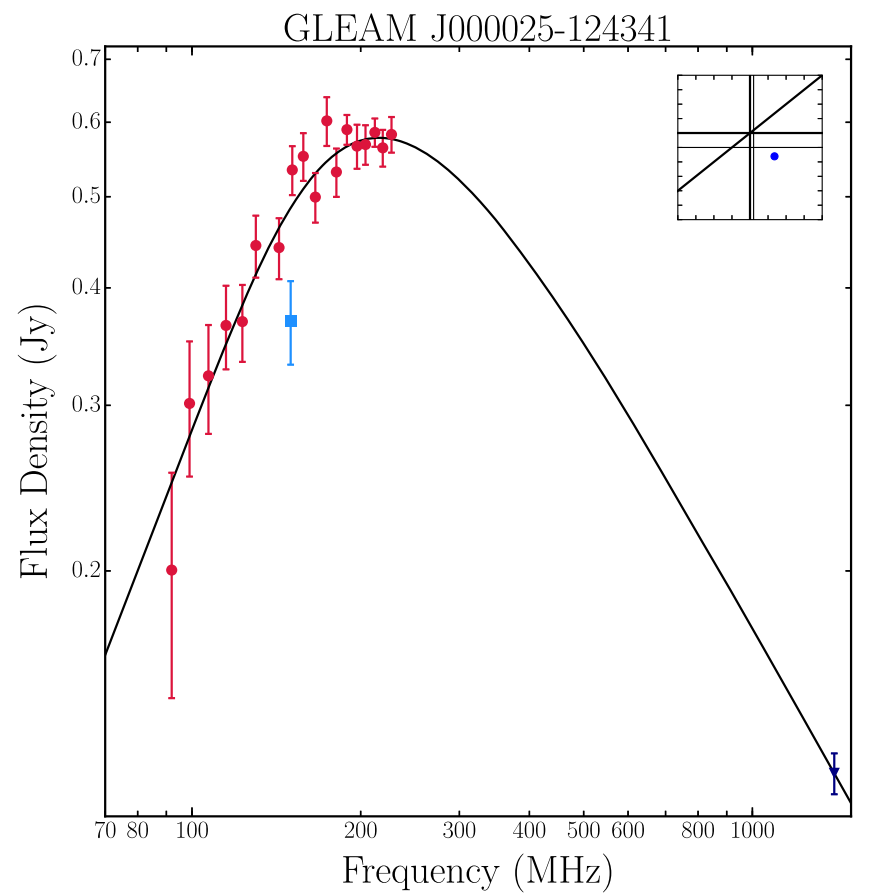

Figure 25. Spectrum of GLEAM J000025-124341 from the high- and lowfrequency peaked-spectrum sample. The purple upward-pointing triangle, red circles, blue square, green leftward-pointing triangle, brown rightward-pointing triangle, and navy downward-pointing triangle represent data points from VLSSr, GLEAM, TGSS-ADR1, MRC, SUMSS, and NVSS, respectively. The fit of the generic curved spectral model of Equation (3) to only the GLEAM, MRC, NVSS, and/or SUMSS flux density points is shown by the black curve. The plot inset in the top right corner displays the position of the source in the color-color diagram of Figure 2 using a blue circle.

(The complete figure set (1519 images) is available.)

wideband image, as detailed in Section 5.3 of this paper. In all cases, the GLEAM survey and NVSS/SUMSS data are presented. If the source also has a MRC, TGSS-ARD1, or VLSSr counterpart, as assessed using the cross-matching routine detailed in Section 3, the flux density from the respective survey is also presented. Overplotted on each spectrum is the best fit of the power-law model of Equation (1) to only the GLEAM, MRC, NVSS, and/or SUMSS data.

In all figure set components the location of the source in the color-color phase space of Figure 2 is shown by a blue circle in the inset plot.

\section{References}

Abazajian, K., Adelman-McCarthy, J. K., Agüeros, M. A., et al. 2004, AJ, 128,502

Adelman-McCarthy, J. K., Agüeros, M. A., Allam, S. S., et al. 2006, ApJS, 162,38

Adelman-McCarthy, J. K., Agüeros, M. A., Allam, S. S., et al. 2008, ApJS, 175,297

An, T., \& Baan, W. A. 2012, ApJ, 760, 77

An, T., Wu, F., Yang, J., et al. 2012, ApJS, 198, 5

Astropy Collaboration, Robitaille, T. P., Tollerud, E. J., et al. 2013, A\&A, 558, A33

Athreya, R. M., \& Kapahi, V. K. 1998, JApA, 19, 63

Baars, J. W. M., Genzel, R., Pauliny-Toth, I. I. K., \& Witzel, A. 1977, A\&A, 61,99

Bannister, K. W., Stevens, J., Tuntsov, A. V., et al. 2016, Sci, 351, 354

Barnes, D. G., Staveley-Smith, L., de Blok, W. J. G., et al. 2001, MNRAS, 322,486

Baum, S. A., O’Dea, C. P., Murphy, D. W., \& de Bruyn, A. G. 1990, A\&A, 232,19
Bell, M. E., Murphy, T., Johnston, S., et al. 2016, MNRAS, 461, 908 Bell, M. E., Murphy, T., Kaplan, D. L., et al. 2014, MNRAS, 438, 352 Bergeron, J., Boissé, P., \& Ménard, B. 2011, A\&A, 525, A51 Best, P. N., \& Heckman, T. M. 2012, MNRAS, 421, 1569

Best, P. N., Peacock, J. A., Brookes, M. H., et al. 2003, MNRAS, 346, 1021 Best, P. N., Röttgering, H. J. A., \& Lehnert, M. D. 1999, MNRAS, 310, 223 Bicknell, G. V., Dopita, M. A., \& O’Dea, C. P. O. 1997, ApJ, 485, 112 Blundell, K. M., Rawlings, S., \& Willott, C. J. 1999, AJ, 117, 677

Bock, D. C.-J., Large, M. I., \& Sadler, E. M. 1999, AJ, 117, 1578

Brienza, M., Godfrey, L., Morganti, R., et al. 2016, A\&A, 585, A29

Briggs, D. S. 1995, PhD thesis, New Mexico Institute of Mining and Technology

Broderick, J. W., Bryant, J. J., Hunstead, R. W., Sadler, E. M., \& Murphy, T. 2007, MNRAS, 381, 341

Brookes, M. H., Best, P. N., Peacock, J. A., Röttgering, H. J. A., \& Dunlop, J. S. 2008, MNRAS, 385, 1297

Browne, I. W. A., Savage, A., \& Bolton, J. G. 1975, MNRAS, 173, 87

Bryant, J. J., Johnston, H. M., Broderick, J. W., et al. 2009, MNRAS, 395, 1099

Budavári, T., \& Szalay, A. S. 2008, ApJ, 679, 301

Burgess, A. M., \& Hunstead, R. W. 2006, AJ, 131, 114

Callingham, J. R., Gaensler, B. M., Ekers, R. D., et al. 2015, ApJ, 809, 168

Cannon, R., Drinkwater, M., Edge, A., et al. 2006, MNRAS, 372, 425

Carilli, C. L., Menten, K. M., Reid, M. J., Rupen, M. P., \& Yun, M. S. 1998 ApJ, 494, 175

Carroll, P. A., Line, J., Morales, M. F., et al. 2016, MNRAS, 461, 4151

Cersosimo, J. C., Santos, M. L., Cintron, S. I., \& Quiniento, Z. M. 1994, ApJS, 95,157

Chand, H., \& Gopal-Krishna 2012, ApJ, 754, 38

Chhetri, R., Ekers, R. D., Jones, P. A., \& Ricci, R. 2013, MNRAS, 434, 956

Chhetri, R., Ekers, R. D., Mahony, E. K., et al. 2012, MNRAS, 422, 2274

Chu, Y.-Q., Zhu, X.-F., \& Butcher, H. 1986, ChA\&A, 10, 196

Colless, M., Dalton, G., Maddox, S., et al. 2001, MNRAS, 328, 1039

Condon, J. J., Cotton, W. D., Greisen, E. W., et al. 1998, AJ, 115, 1693

Coppejans, R., Cseh, D., van Velzen, S., et al. 2016, MNRAS, 459, 2455

Coppejans, R., Cseh, D., Williams, W. L., van Velzen, S., \& Falcke, H. 2015 MNRAS, 450, 1477

Croom, S. M., Smith, R. J., Boyle, B. J., et al. 2004, MNRAS, 349, 1397

Dallacasa, D., Stanghellini, C., Centonza, M., \& Fanti, R. 2000, A\&A 363,887

De Breuck, C., Klamer, I., Johnston, H., et al. 2006, MNRAS, 366, 58

De Breuck, C., Tang, Y., de Bruyn, A. G., Röttgering, H., \& van Breugel, W. 2002, A\&A, 394, 59

De Breuck, C., van Breugel, W., Röttgering, H., et al. 2001, AJ, 121, 1241

de Vries, N., Snellen, I. A. G., Schilizzi, R. T., Lehnert, M. D., \& Bremer, M. N. 2007, A\&A, 464, 879

de Vries, W. H., Barthel, P. D., \& Hes, R. 1995, A\&AS, 114, 259

de Vries, W. H., Barthel, P. D., \& O'Dea, C. P. 1997, A\&A, 321, 105

de Vries, W. H., O'Dea, C. P., Barthel, P. D., \& Thompson, D. J. 2000, A\&AS, 143,181

de Vries, W. H., O’Dea, C. P., Perlman, E., et al. 1998, ApJ, 503, 138

di Serego-Alighieri, S., Danziger, I. J., Morganti, R., \& Tadhunter, C. N. 1994, MNRAS, 269, 998

Douglas, J. N., Bash, F. N., Bozyan, F. A., Torrence, G. W., \& Wolfe, C. 1996, AJ, 111, 1945

Down, E. J., Rawlings, S., Sivia, D. S., \& Baker, J. C. 2010, MNRAS, 401,633

Drake, C. L., McGregor, P. J., Dopita, M. A., \& van Breugel, W. J. M. 2003 AJ, 126, 2237

Drinkwater, M. J., Jurek, R. J., Blake, C., et al. 2010, MNRAS, 401, 1429

Drinkwater, M. J., Webster, R. L., Francis, P. J., et al. 1997, MNRAS, 284, 85

Duffy, P., \& Blundell, K. M. 2012, MNRAS, 421, 108

Edge, D. O., Shakeshaft, J. R., McAdam, W. B., Baldwin, J. E., \& Archer, S. 1959, MmRAS, 68, 37

Edwards, P. G., \& Tingay, S. J. 2004, A\&A, 424, 91

Ekers, J. A. 1969, AuJPA, 7, 3

Ellison, S. L., Prochaska, J. X., Hennawi, J., et al. 2010, MNRAS, 406, 1435

Ellison, S. L., Yan, L., Hook, I. M., et al. 2001, A\&A, 379, 393

Falcke, H., Körding, E., \& Nagar, N. M. 2004, NewAR, 48, 1157

Fanaroff, B. L., \& Riley, J. M. 1974, MNRAS, 167, 31P

Fanti, R., Fanti, C., Schilizzi, R. T., et al. 1990, A\&A, 231, 333

Farnes, J. S., O’Sullivan, S. P., Corrigan, M. E., \& Gaensler, B. M. 2014, ApJ, 795, 63

Fiedler, R., Dennison, B., Johnston, K. J., Waltman, E. B., \& Simon, R. S 1994, ApJ, 430, 581

Fisher, K. B., Huchra, J. P., Strauss, M. A., et al. 1995, ApJS, 100, 69 
Foreman-Mackey, D., Hogg, D. W., Lang, D., \& Goodman, J. 2013, PASP, 125,306

Franzen, T. M. O., Jackson, C. A., Offringa, A. R., et al. 2016, MNRAS, 459, 3314

Fricke, K. J., Kollatschny, W., \& Witzel, A. 1983, A\&A, 117, 60

Gendre, M. A., \& Wall, J. V. 2008, MNRAS, 390, 819

Goodman, J., \& Weare, J. 2010, Commun. Appl. Math. Comput. Sci., 5, 65

Gregory, P. C., \& Condon, J. J. 1991, ApJS, 75, 1011

Gregory, P. C., Vavasour, J. D., Scott, W. K., \& Condon, J. J. 1994, ApJS, 90,173

Gugliucci, N. E., Taylor, G. B., Peck, A. B., \& Giroletti, M. 2005, ApJ, 622,136

Hales, S. E. G., Riley, J. M., Waldram, E. M., Warner, P. J., \& Baldwin, J. E. 2007, MNRAS, 382, 1639

Halpern, J. P., \& Eracleous, M. 1997, IAUC, 6639, 2

Halpern, J. P., Eracleous, M., \& Mattox, J. R. 2003, AJ, 125, 572

Hammond, A. M., Robishaw, T., \& Gaensler, B. M. 2012, arXiv:1209.1438

Hancock, P. J., Murphy, T., Gaensler, B. M., Hopkins, A., \& Curran, J. R. 2012, MNRAS, 422, 1812

Hancock, P. J., Sadler, E. M., Mahony, E. K., \& Ricci, R. 2010, MNRAS, 408, 1187

Heald, G. H., Pizzo, R. F., Orrú, E., et al. 2015, A\&A, 582, A123

Healey, S. E., Romani, R. W., Cotter, G., et al. 2008, ApJS, 175, 97

Heckman, T. M., O’Dea, C. P., Baum, S. A., \& Laurikainen, E. 1994, ApJ, 428, 65

Heidt, J., Tröller, M., Nilsson, K., et al. 2004, A\&A, 418, 813

Helou, G., Madore, B. F., Schmitz, M., et al. 1991, in Astrophysics and Space Science Library 171, Databases and On-line Data in Astronomy, ed. M. A. Albrecht \& D. Egret (Amsterdam: Springer), 89

Hewett, P. C., \& Wild, V. 2010, MNRAS, 405, 2302

Hewitt, A., \& Burbidge, G. 1987, ApJS, 63, 1

Hewitt, A., \& Burbidge, G. 1989, ApJS, 69, 1

Hewitt, A., \& Burbidge, G. 1991, ApJS, 75, 297

Hinshaw, G., Larson, D., Komatsu, E., et al. 2013, ApJS, 208, 19

Hogan, M. T., Edge, A. C., Geach, J. E., et al. 2015, MNRAS, 453, 1223

Holt, J., Tadhunter, C. N., \& Morganti, R. 2008, MNRAS, 387, 639

Hunstead, R. W., Murdoch, H. S., \& Shobbrook, R. R. 1978, MNRAS, 185,149

Hurley-Walker, N., Callingham, J. R., Hancock, P. J., et al. 2017, MNRAS, 464, 1146

Intema, H. T., Jagannathan, P., Mooley, K. P., \& Frail, D. A. 2016, arXiv: 1603.04368

Jackson, C. A., Wall, J. V., Shaver, P. A., et al. 2002, A\&A, 386, 97

Jaffe, W. J., \& Perola, G. C. 1973, A\&A, 26, 423

Jarvis, M. J., Rawlings, S., Eales, S., et al. 2001, MNRAS, 326, 1585

Jauncey, D. L., Batty, M. J., Wright, A. E., Peterson, B. A., \& Savage, A. 1984, ApJ, 286, 498

Jauncey, D. L., Wright, A. E., Peterson, B. A., \& Condon, J. J. 1978, ApJL, 219, L1

Jones, D. H., Read, M. A., Saunders, W., et al. 2009, MNRAS, 399, 683

Jones, D. H., Saunders, W., Colless, M., et al. 2004, MNRAS, 355, 747

Jones, D. L., Tingay, S. J., Murphy, D. W., et al. 1996, ApJL, 466, L63

Kaiser, C. R., \& Alexander, P. 1997, MNRAS, 286, 215

Kameno, S., Horiuchi, S., Shen, Z.-Q., et al. 2000, PASJ, 52, 209

Kapahi, V. K., Athreya, R. M., van Breugel, W., McCarthy, P. J., \& Subrahmanya, C. R. 1998, ApJS, 118, 275

Kardashev, N. S. 1962, SvA, 6, 317

Kempner, J. C., Blanton, E. L., Clarke, T. E., et al. 2004, in The Riddle of Cooling Flows in Galaxies and Clusters of Galaxies, ed. T. Reiprich, J. Kempner, \& N. Soker (arXiv:0310263)

Klamer, I. J., Ekers, R. D., Bryant, J. J., et al. 2006, MNRAS, 371, 852

Krichbaum, T. P., Alef, W., Witzel, A., et al. 1998, A\&A, 329, 873

Kuncic, Z., Bicknell, G. V., \& Dopita, M. A. 1998, ApJL, 495, L35

Kunert-Bajraszewska, M., Gawroński, M. P., Labiano, A., \& Siemiginowska, A. 2010, MNRAS, 408, 2261

Labiano, A., Barthel, P. D., O’Dea, C. P., et al. 2007, A\&A, 463, 97

Lane, W. M., Cotton, W. D., van Velzen, S., et al. 2014, MNRAS, 440, 327

Large, M. I., Cram, L. E., \& Burgess, A. M. 1991, Obs, 111, 72

Large, M. I., Mills, B. Y., Little, A. G., Crawford, D. F., \& Sutton, J. M. 1981, MNRAS, 194, 693

Lawrence, C. R., Elston, R., Januzzi, B. T., \& Turner, E. L. 1995, AJ, 110,2570

Lazio, T. J. W., Clarke, T. E., Lane, W. M., et al. 2010, AJ, 140, 1995

Liang, E. W., \& Liu, H. T. 2003, MNRAS, 340, 632

Line, J. L. B., Webster, R. L., Pindor, B., Mitchell, D. A., \& Trott, C. M. 2016, arXiv:1611.05534
Lobanov, A. P. 1998, A\&A, 330, 79

Mahony, E. K., Morganti, R., Prandoni, I., et al. 2016, MNRAS, 463, 2997

Mahony, E. K., Sadler, E. M., Croom, S. M., et al. 2011, MNRAS, 417, 2651

Manchester, R. N., Hobbs, G. B., Teoh, A., \& Hobbs, M. 2005, AJ, 129, 1993

Marcha, M. J. M., Browne, I. W. A., Impey, C. D., \& Smith, P. S. 1996, MNRAS, 281, 425

Marr, J. M., Perry, T. M., Read, J., Taylor, G. B., \& Morris, A. O. 2014, ApJ, 780, 178

Marr, J. M., Taylor, G. B., \& Crawford, F., III 2001, ApJ, 550, 160

Massardi, M., Ekers, R. D., Murphy, T., et al. 2011, MNRAS, 412, 318

Massaro, E., Maselli, A., Leto, C., et al. 2015, Ap\&SS, 357, 75

Mauch, T., Murphy, T., Buttery, H. J., et al. 2003, MNRAS, 342, 1117

Mauch, T., \& Sadler, E. M. 2007, MNRAS, 375, 931

Maza, J., Ortiz, P. F., Wischnjewsky, M., Antezana, R., \& González, L. E. 1995a, RMxAA, 31, 159

Maza, J., Wischnjewsky, M., Antezana, R., \& González, L. E. 1995b, RMxAA, 31,119

McCarthy, P. J., Kapahi, V. K., van Breugel, W., et al. 1996, ApJS, 107, 19

McGilchrist, M. M., \& Riley, J. M. 1990, MNRAS, 246, 123

Meisner, A. M., \& Romani, R. W. 2010, ApJ, 712, 14

Melrose, D. B. 1971, Ap\&SS, 12, 172

Mills, B. Y. 1981, PASAu, 4, 156

Mills, B. Y., Slee, O. B., \& Hill, E. R. 1958, AuJPh, 11, 360

Mills, B. Y., Slee, O. B., \& Hill, E. R. 1960, AuJPh, 13, 676

Mills, B. Y., Slee, O. B., \& Hill, E. R. 1961, AuJPh, 14, 497

Murdoch, H. S., Crawford, D. F., \& Jauncey, D. L. 1973, ApJ, 183, 1

Murgia, M. 2003, PASA, 20, 19

Murgia, M., Fanti, C., Fanti, R., et al. 1999, A\&A, 345, 769

Murphy, T., Sadler, E. M., Ekers, R. D., et al. 2010, MNRAS, 402, 2403

Nelder, J. A., \& Mead, R. 1965, CompJ, 7, 308

Noterdaeme, P., Srianand, R., \& Mohan, V. 2010, MNRAS, 403, 906

O'Dea, C. P. 1998, PASP, 110, 493

O’Dea, C. P., \& Baum, S. A. 1997, AJ, 113, 148

O’Dea, C. P., Baum, S. A., \& Stanghellini, C. 1991, ApJ, 380, 66

Offringa, A. R., McKinley, B., Hurley-Walker, N., et al. 2014, MNRAS, 444,606

Offringa, A. R., van de Gronde, J. J., \& Roerdink, J. B. T. M. 2012, A\&A, 539, A95

Offringa, A. R., Wayth, R. B., Hurley-Walker, N., et al. 2015, PASA, 32, 8

Orienti, M., \& Dallacasa, D. 2008, A\&A, 487, 885

Orienti, M., Dallacasa, D., Tinti, S., \& Stanghellini, C. 2006, A\&A, 450, 959

Orienti, M., Murgia, M., \& Dallacasa, D. 2010, MNRAS, 402, 1892

Osmer, P. S., Porter, A. C., \& Green, R. F. 1994, ApJ, 436, 678

Owsianik, I., \& Conway, J. E. 1998, A\&A, 337, 69

Pacholczyk, A. G. 1970, Radio Astrophysics. Nonthermal Processes in Galactic and Extragalactic Sources (New York: W.H.Freeman \& Co)

Peck, A. B., \& Taylor, G. B. 2000, ApJ, 534, 90

Peck, A. B., Taylor, G. B., \& Conway, J. E. 1999, ApJ, 521, 103

Phillips, R. B., \& Mutel, R. L. 1980, ApJ, 236, 89

Pilkington, J. D. H., \& Scott, J. F. 1965, MmRAS, 69, 183

Polatidis, A. G., \& Conway, J. E. 2003, PASA, 20, 69

Randall, K. E., Hopkins, A. M., Norris, R. P., \& Edwards, P. G. 2011, MNRAS, 416, 1135

Rasmussen, C., \& Williams, C. 2006, Gaussian Processes for Machine Learning, Adaptive Computation and Machine Learning (Cambridge, MA: MIT Press), 248

Readhead, A. C. S., Taylor, G. B., Xu, W., et al. 1996, ApJ, 460, 612

Rengelink, R. B., Tang, Y., de Bruyn, A. G., et al. 1997, A\&AS, 124, 259

Reuland, M., van Breugel, W., Röttgering, H., et al. 2003, ApJ, 592, 755

Richards, G. T., Myers, A. D., Gray, A. G., et al. 2009, ApJS, 180, 67

Robertson, J. G. 1991, AuJPh, 44, 729

Roettgering, H. J. A., van Ojik, R., Miley, G. K., et al. 1997, A\&A, 326, 505

Rowlinson, A., Bell, M. E., Murphy, T., et al. 2016, MNRAS, 458, 3506

Rupke, D. S., Veilleux, S., \& Sanders, D. B. 2005, ApJ, 632, 751

Sadler, E. M., Ricci, R., Ekers, R. D., et al. 2006, MNRAS, 371, 898

Schneider, D. P., Richards, G. T., Hall, P. B., et al. 2010, AJ, 139, 2360

Singal, A. K. 1993, MNRAS, 263, 139

Slee, O. B. 1995, AuJPh, 48, 143

Snellen, I. A. G., Bremer, M. N., Schilizzi, R. T., Miley, G. K., \& van Ojik, R. 1996, MNRAS, 279, 1294

Snellen, I. A. G., Lehnert, M. D., Bremer, M. N., \& Schilizzi, R. T. 2002 MNRAS, 337, 981

Snellen, I. A. G., Schilizzi, R. T., de Bruyn, A. G., et al. 1998, A\&AS, 131, 435

Snellen, I. A. G., Schilizzi, R. T., Miley, G. K., et al. 2000, MNRAS, 319, 445

Sowards-Emmerd, D., Romani, R. W., Michelson, P. F., Healey, S. E., \& Nolan, P. L. 2005, ApJ, 626, 95 
Sowards-Emmerd, D., Romani, R. W., Michelson, P. F., \& Ulvestad, J. S. 2004, ApJ, 609, 564

Stanghellini, C., O'Dea, C. P., Baum, S. A., et al. 1997, A\&A, 325, 943

Stanghellini, C., O’Dea, C. P., Dallacasa, D., et al. 1998, A\&AS, 131, 303

Stern, D., Dey, A., Spinrad, H., et al. 1999, AJ, 117, 1122

Stickel, M., Fried, J. W., \& Kuehr, H. 1989, A\&AS, 80, 103

Stickel, M., Meisenheimer, K., \& Kuehr, H. 1994, A\&AS, 105, 211

Stickel, M., Rieke, G. H., Kuehr, H., \& Rieke, M. J. 1996, ApJ, 468, 556

Swarup, G. 1991, in ASP Conf. Ser. 19, IAU Coll. 131: Radio Interferometry. Theory, Techniques, and Applications, ed. T. J. Cornwell \& R. A. Perley (San Francisco, CA: ASP), 376

Tadhunter, C. N., Morganti, R., di Serego-Alighieri, S., Fosbury, R. A. E., \& Danziger, I. J. 1993, MNRAS, 263, 999

Tadhunter, C. N., Ramos Almeida, C., Morganti, R., et al. 2012, MNRAS, 427, 1603

Taylor, M. B. 2005, in ASP Conf. Ser. 347, Astronomical Data Analysis Software and Systems XIV, ed. P. Shopbell, M. Britton, \& R. Ebert (San Francisco, CA: ASP), 29

Thompson, D. J., Djorgovski, S., \& de Carvalho, R. 1990, PASP, 102, 1235

Tingay, S. J., \& de Kool, M. 2003, AJ, 126, 723

Tingay, S. J., Goeke, R., Bowman, J. D., et al. 2013, PASA, 30, 7

Tingay, S. J., Macquart, J.-P., Collier, J. D., et al. 2015, AJ, 149, 74

Tingay, S. J., \& Murphy, D. W. 2001, ApJ, 546, 210

Tinti, S., Dallacasa, D., de Zotti, G., Celotti, A., \& Stanghellini, C. 2005, A\&A, 432,31

Tinti, S., \& de Zotti, G. 2006, A\&A, 445, 889
Titov, O., Jauncey, D. L., Johnston, H. M., Hunstead, R. W., \& Christensen, L. 2011, AJ, 142, 165

Tonry, J. L., \& Kochanek, C. S. 1999, AJ, 117, 2034

Torniainen, I., Tornikoski, M., Lähteenmäki, A., et al. 2007, A\&A, 469, 451

van Breugel, W., De Breuck, C., Stanford, S. A., et al. 1999, ApJL, 518, L61

van Breugel, W., Miley, G., \& Heckman, T. 1984, AJ, 89, 5

van Haarlem, M. P., Wise, M. W., Gunst, A. W., et al. 2013, A\&A, 556, A2

Walker, R. C., Romney, J. D., \& Benson, J. M. 1994, ApJL, 430, L45

Wall, J. V., \& Peacock, J. A. 1985, MNRAS, 216, 173

Wayth, R. B., Lenc, E., Bell, M. E., et al. 2015, PASA, 32, 25

Wen, Z. L., Han, J. L., \& Liu, F. S. 2010, ApJS, 187, 272

Wiklind, T., \& Combes, F. 1997, A\&A, 328, 48

Wilkinson, P. N., Polatidis, A. G., Readhead, A. C. S., Xu, W., \& Pearson, T. J. 1994, ApJL, 432, L87

Willott, C. J., Rawlings, S., \& Jarvis, M. J. 2000, MNRAS, 313, 237

Wright, A., \& Otrupcek, R. 1990, in PKS Catalog (Australia Telescope National Facility)

Wright, A. E., Ables, J. G., \& Allen, D. A. 1983, MNRAS, 205, 793

Wright, A. E., Griffith, M. R., Burke, B. F., \& Ekers, R. D. 1994, ApJS, 91, 111

Wright, A. E., Peterson, B. A., Jauncey, D. L., \& Condon, J. J. 1979, ApJ, 229, 73

York, D. G., Adelman, J., Anderson, J. E., Jr., et al. 2000, AJ, 120, 1579

Zafar, T., Popping, A., \& Péroux, C. 2013, A\&A, 556, A140

Zickgraf, F.-J., Voges, W., Krautter, J., et al. 1997, A\&A, 323, L21 